\title{
Role of stromal SPARC in PDAC tumorigenesis and drug delivery
}

\author{
Dissertation \\ for the award of the degree \\ "Doctor of Philosophy (Ph.D)" \\ of the Georg-August-Universität Göttingen \\ within the doctoral program Molecular medicine, \\ of the Georg-August University School of Science (GAUSS)
}

Submitted by
Iswarya Ramu

Born in

Velivayal, India

Göttingen, 2018 


\section{$\underline{\text { Thesis Supervisor }}$}

PD Dr. Dr. med. Albrecht Neesse

\section{Members of the thesis advisory committee}

PD Dr. Dr. med. Albrecht Neesse (Reviewer)

Department of Gastroenterology and Gastrointestinal Oncology

University Medical center Göttingen

Prof. Dr. Steven Johnsen (Reviewer)

Department of General, Visceral and Pediatric Surgery

University Medical center Göttingen

Prof. Dr. Matthias Dobbelstein

Department of Molecular Oncology

University Medical center Göttingen

\section{Further members of the examination board}

Prof. Dr. med. Frauke Alves

Department of Hematology and Oncology, University Medical Center Göttingen

Prof. Dr. Heidi Hahn

Department of Human Genetics, University Medical Center Göttingen

PD Dr. rer. nat. Laura Zelarayan-Behrend

Institute of Pharmacology and Toxicology, University Medical Center Göttingen

Date of oral examination: 10.12.2018 


\section{Affidavit}

I hereby declare that the $\mathrm{PhD}$ thesis entitled "Role of stromal SPARC in PDAC tumorigenesis and drug delivery" has been written independently and with no other sources and aids than quoted.

\section{Iswarya Ramu}

October, 2018

Göttingen 


\section{Table of Contents}

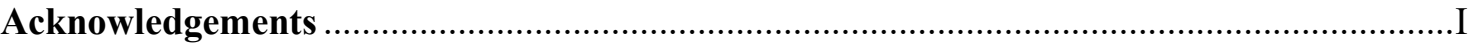

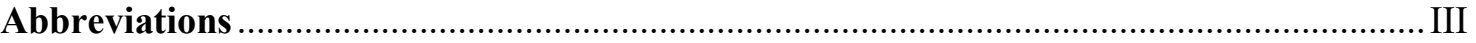

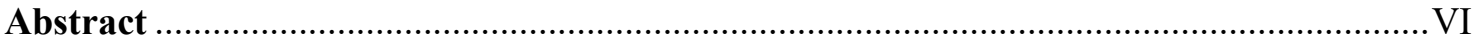

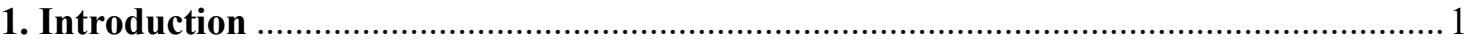

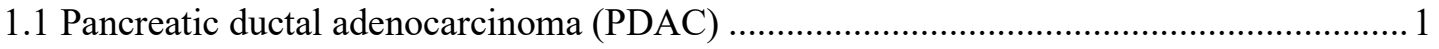

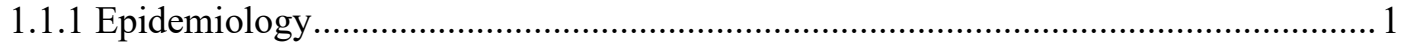

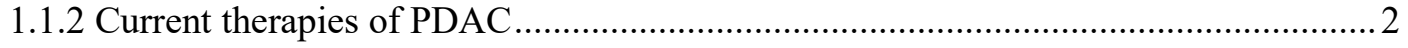

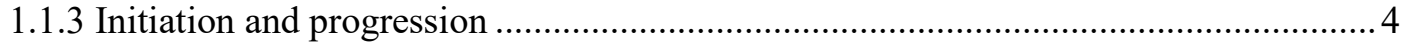

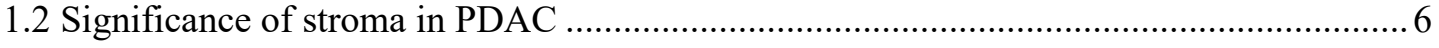

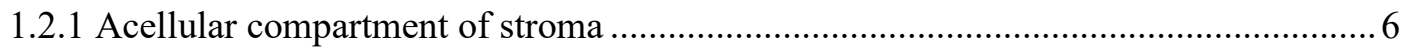

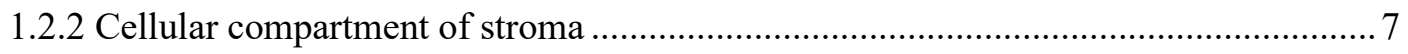

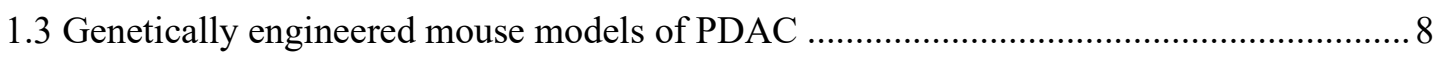

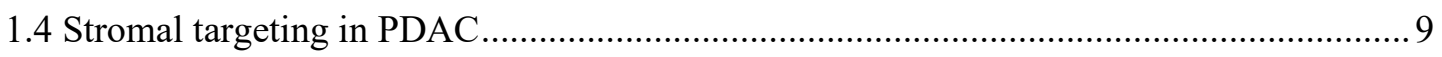

1.5 Secreted Protein Acidic Rich in Cysteine (SPARC).................................................... 11

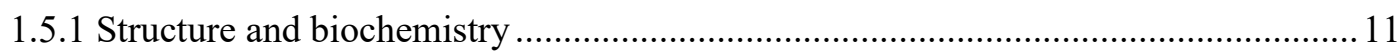

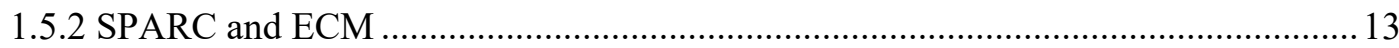

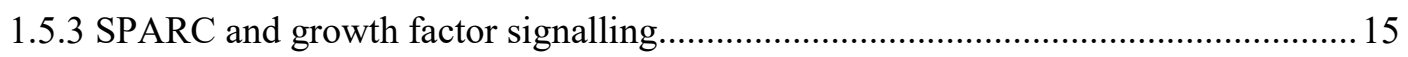

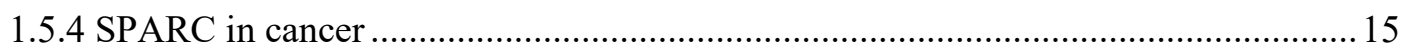

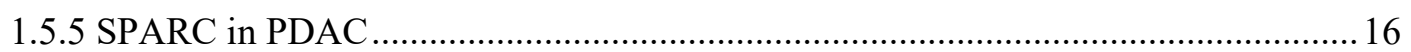

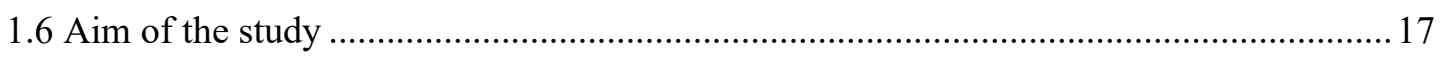

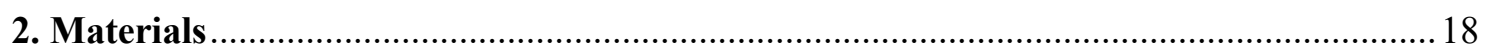

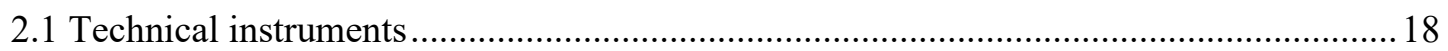

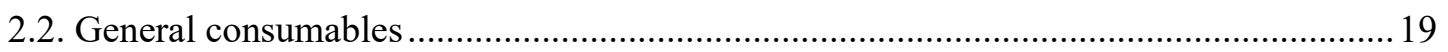

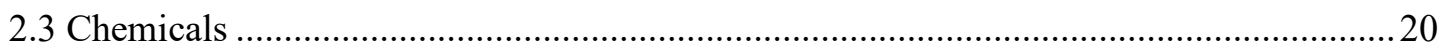

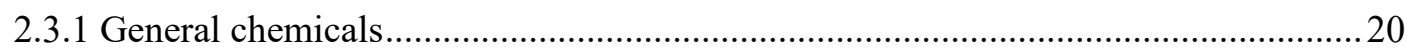

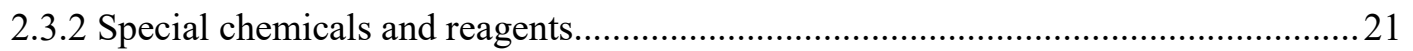

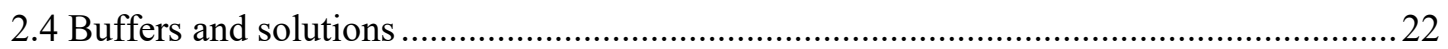

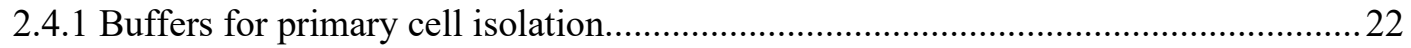

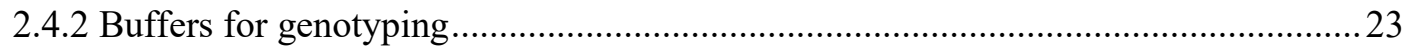

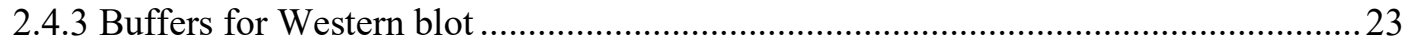

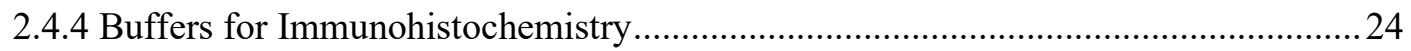

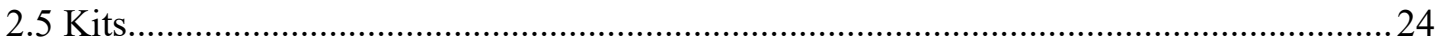

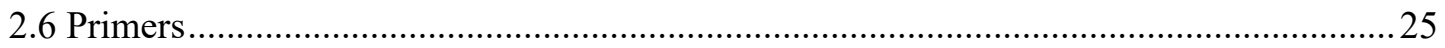

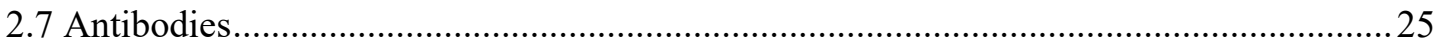




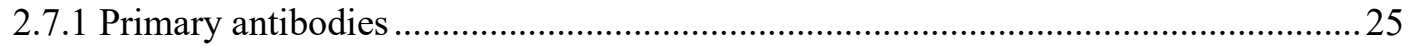

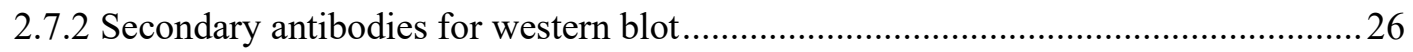

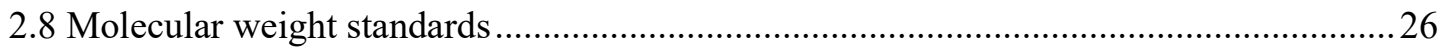

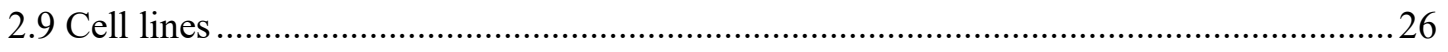

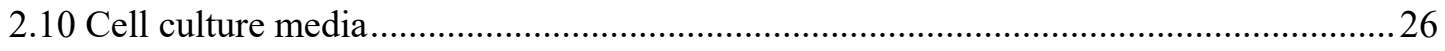

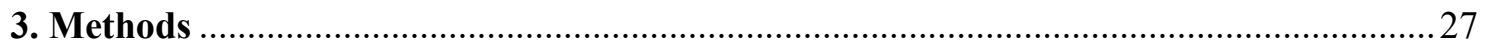

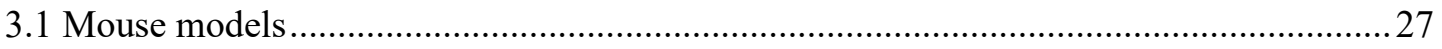

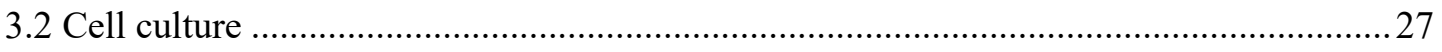

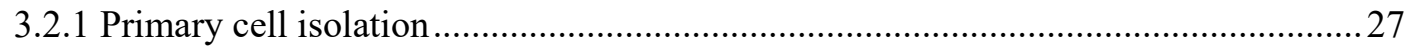

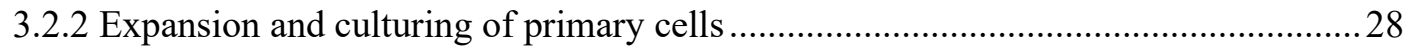

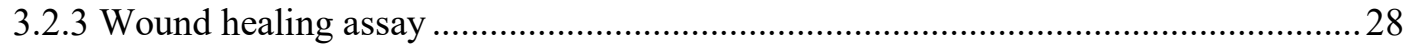

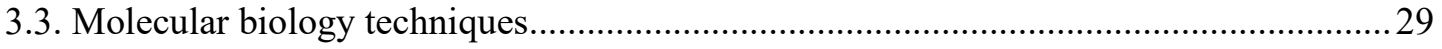

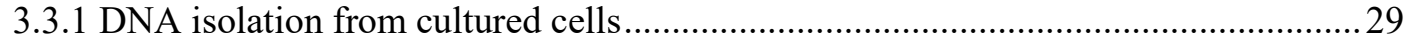

3.3.2 DNA isolation from mouse tails for genotyping....................................................2 29

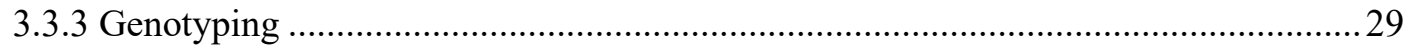

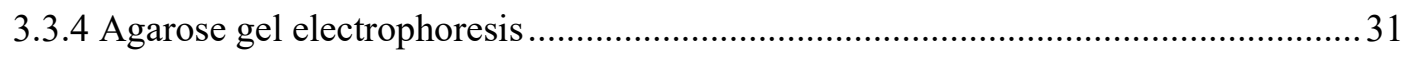

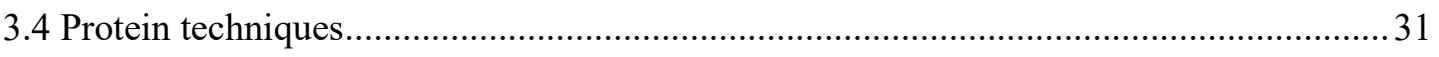

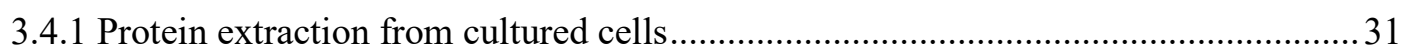

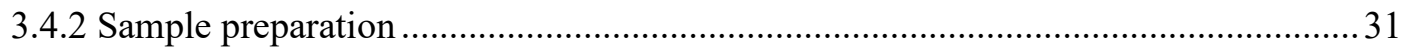

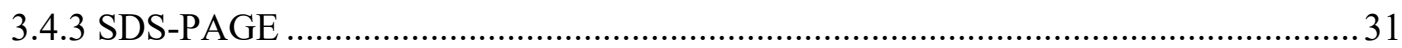

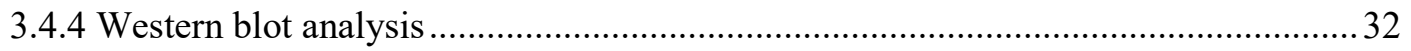

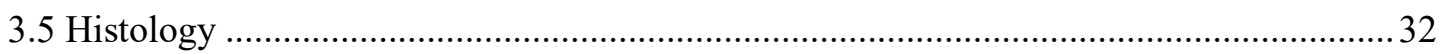

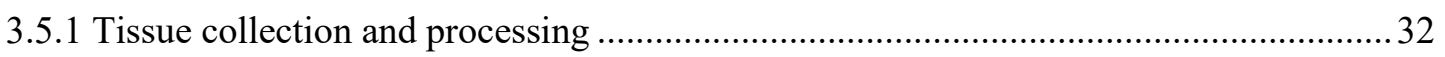

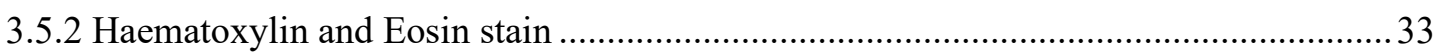

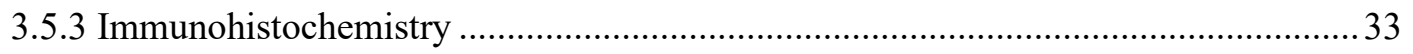

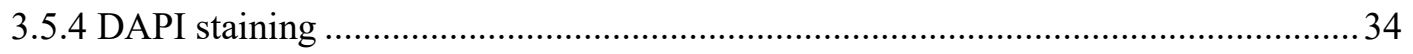

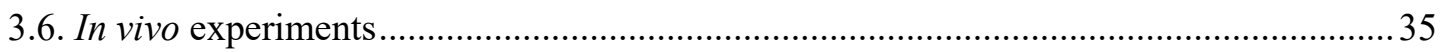

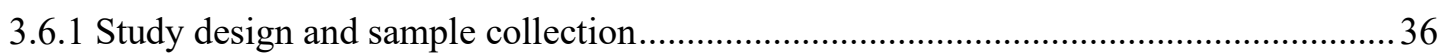

3.6.2 Gemcitabine treatment and the sample preparation for Mass Spectrometry analysis ...36

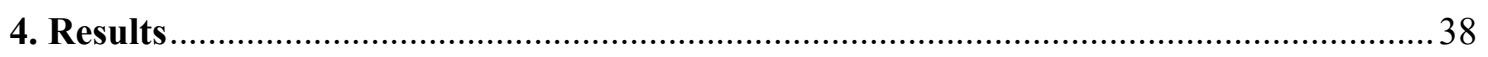

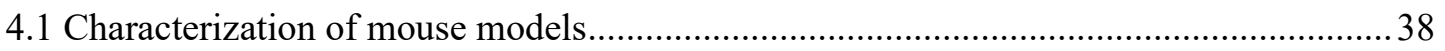

4.1.1 SPARC knock out does not affect murine pancreas development.............................40

4.1.2 Oncogenic $\mathrm{Kras}^{\mathrm{G} 12 \mathrm{D}}$ activation results in disease initiation ................................... 40

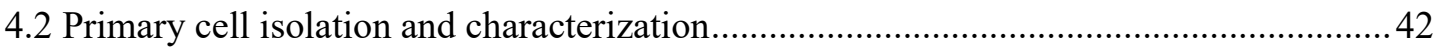

4.2.1 Characterization of PAF cells by 1LoxP Kras genotype ...........................................43

4.2.2 Characterization of PanIN cells by western blot........................................................ 43 


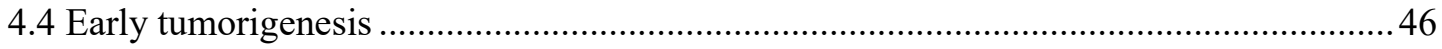

4.4.1 SPARC depletion does not affect ADMs, PanIN development and inflammation ... 46

4.4.2 Collagen deposition is significantly reduced upon SPARC depletion.........................47

4.4.3 SPARC depletion does not affect fibroblast population .............................................49

4.4.4 SPARC depletion does not affect overall proliferation in vivo...................................50

4.4.5 Stroma derived SPARC does not affect migration of PanIN cells ............................5 52

4.4.6 SPARC and angiogenesis during early and late tumorigenesis .................................54

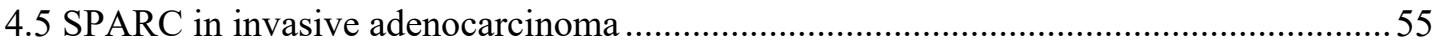

4.5.1 SPARC status does not affect the tumor incidence and the tumor weight ................55

4.5.2 Collagen deposition was impaired upon SPARC depletion in established tumors....56

4.5.3 SPARC and hyaluronic acid deposition in tumor ....................................................5

4.5.4 SPARC depletion does not affect fibroblast density in established tumor ................58

4.5.5 Tumor proliferation and apoptosis were not affected by stromal SPARC ................59

4.5.6 SPARC status has no correlation with liver metastasis burden ..................................60

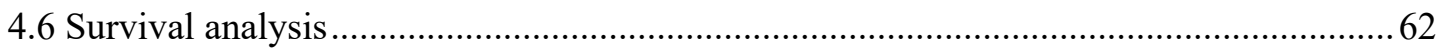

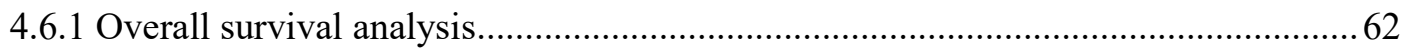

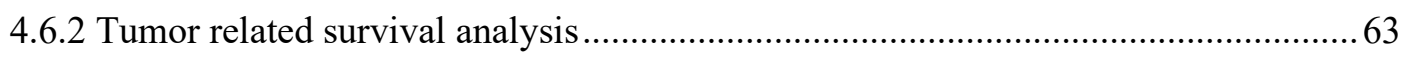

4.6.3 Tumor bearing KC-SPARC ${ }^{-/}$mice show severe tumor related complications ........... 64

4.7 SPARC does not affect gemcitabine delivery and metabolism ......................................65

4.7.1 SPARC mediated collagen deposition does not affect gemcitabine delivery and

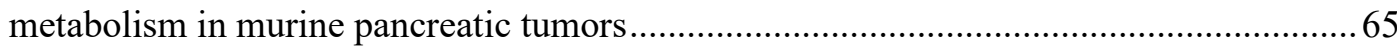

4.7.2 Overall cellularity but not the SPARC mediated collagen deposition affects drug

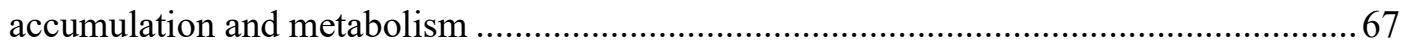

4.7.3 Gemcitabine is enzymatically inactivated by drug metabolizing enzymes ............... 71

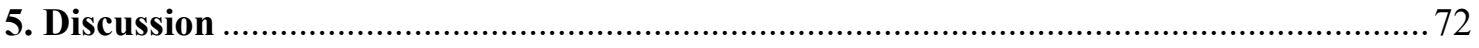

5.1 Advantages and disadvantages of genetically engineered mice used in this study ...........72

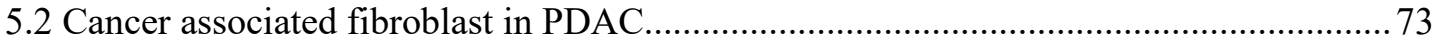

5.3 SPARC mediated ECM deposition and PDAC progression.............................................. 75

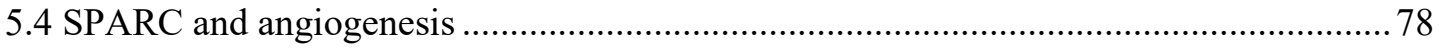

5.5 SPARC mediated desmoplasia and drug delivery …...................................................... 79

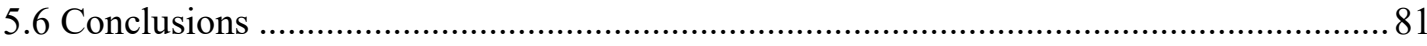

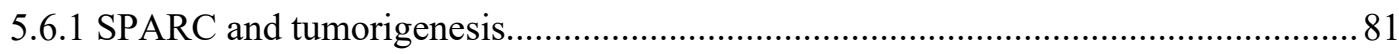

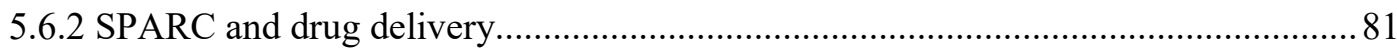

6. References

83 Error! Bookmark not defined. 


\section{Acknowledgements}

With immense gratitude, I take this opportunity to convey my heartfelt thanks to my supervisor PD. Dr. Dr. med. Albrecht Neesse for giving me this chance to work in his research group and for his constant support and motivation throughout the course of my Ph.D project.

I sincerely thank my thesis advisory committee members Prof. Dr. Steven A. Johnsen and Prof. Dr. Matthias Dobbelstein for their support and valuable discussions and suggestions for my project during each thesis committee meeting.

On a special note, I would like to thank Prof. Dr. med. Volker Ellenrieder, PD. Dr. med. Elisabeth Hessmann and Dr. Shiv Singh for providing guidance and discussions about my experiments and for helping with any critical situations. I deeply thank our former colleague Dr. Nai-Ming Chen for teaching the techniques during the initial days of my thesis project.

I am grateful to Prof. Dr. med. Bence Sipos and Prof. Dr. Phillipp Ströbel for their contribution in histological analysis and tumor grading. I would like to sincerely thank Prof. Duncan Jodrell and Dr. Frances Richards for helping us with the pharmacokinetic analysis.

I thank my fellow Ph.D student Melanie Patzak for helping me with some of the mouse experiments and for being available if I needed any help. I also would like to thank the fellow medical dissertation students Lukas Klein and Sören Buchholz for their moral support during the course of my thesis project. I would like to convey my deep thanks to Dr. Robert Goetze and Laurin Wolf for standing at my side in every situation and for their brotherly love and support. I would like to extend my sincere thanks to our talented and hardworking technical assistants Jutta Blumberg and Ulrike Wegner for their constant support and contribution throughout my project.

I thank my fellow Ph.D students Shilpa Patil, Umair Latif, Zhe Zhang (Alice), Mengyu Tu, Marie Hasselluhn, Geske Schmidt, Lennart Versemann, Kevin Weimer, Lina Frank and other technical assistants of our department for creating such a wonderful work atmosphere and for the fruitful scientific discussions regarding my thesis and in general. 
I would like to convey a special thanks to my best friend Veera for giving me the moral support throughout the course of my stay in Germany. I am truly grateful to my best friends Ari, Rajeev, Brindha, Shilpa and Maithy for filling me up with the positive energy whenever I was drained.

I would not have survived this long and challenging journey without the constant support from my family. Therefore, I would like dedicate my $\mathrm{Ph} . \mathrm{D}$ thesis to my family. I would like to thank my mother Mrs. Pazhaniammal Ramu, my sisters Mrs. Sathya Krishnaraj and Sonu and my brother in law Dr. K. Krishnaraj for being my back bone. I like to thank my sister Dr. Sankari Nagarajan for being the inspiration and for her constant support and guidance throughout my career.

At last but most importantly I wish to thank my husband Vino who inspires me greatly with his enthusiastic nature and whose presence influences me positively. Thank you for being who you are. Your efforts and contribution can't be described in words, I am deeply thankful for all your support. 


\section{Abbreviations}

\begin{tabular}{|c|c|}
\hline$\alpha$ & alpha \\
\hline$\beta$ & beta \\
\hline$\mu$ & micro \\
\hline$\mu l$ & microliter \\
\hline$\mu \mathrm{m}$ & micrometre \\
\hline$\mu \mathrm{M}$ & micromole \\
\hline${ }^{\circ} \mathrm{C}$ & degree celsius \\
\hline $2 \mathrm{D}$ & two dimensional \\
\hline aa & aminoacid \\
\hline $\mathrm{ABC}$ & avidin-biotin Complex \\
\hline $\mathrm{ADM}$ & acinar to ductal metaplasia \\
\hline asn & asparagine \\
\hline$\alpha-\mathrm{SMA}$ & alpha-Smooth Muscle Actin \\
\hline bp & base pair \\
\hline BM-40 & basement membrane protein- 40 \\
\hline BSA & bovine serum albumin \\
\hline$B R C A 1$ & breast cancer 1 \\
\hline CAFs & cancer associated fibroblasts \\
\hline $\mathrm{CC} 3$ & cleaved caspase 3 \\
\hline CCL & chemokine (C-C motif) Ligand \\
\hline CD-31 & cluster of differentiation 31 \\
\hline $\mathrm{CDA}$ & cytidine deaminase \\
\hline $\mathrm{CO}_{2}$ & carbon dioxide \\
\hline CTGF & connective tissue growth factor \\
\hline $\mathrm{Da}$ & Dalton \\
\hline DAB & 3,3'-Diaminobenzidine \\
\hline DAPI & diamidino-2-phenylindole \\
\hline $\mathrm{dCK}$ & deoxycytidine kinase \\
\hline DDR2 & discoidin domain receptor 2 \\
\hline $\mathrm{dFdC}$ & 2',2'-difluorodeoxycytidine \\
\hline $\mathrm{dFdCDP}$ & difluorodeoxycytidine diphosphate \\
\hline dFdCMP & difluorodeoxycytidine monophosphate \\
\hline dFdCTP & 2',2'-difluorodeoxycytidine-5'-triphosphate \\
\hline $\mathrm{dFdU}$ & 2',2'-difluorodeoxyuridine \\
\hline DMEM & dulbecco's Modified Eagle's Medium \\
\hline DNA & deoxyribonucleic acid \\
\hline EC domain & extra cellular domain \\
\hline ECM & extra cellular matrix \\
\hline ECL & enhanced chemiluminescence \\
\hline EDTA & ethylenediaminetetraacetic acid \\
\hline EGF & epidermal growth factor \\
\hline EMT & epithelial to mesenchymal transition \\
\hline ER & endoplasmic reticulum \\
\hline ERK & extracellular signal-regulated kinase \\
\hline FBS & fetal bovine serum \\
\hline FGF & fibroblast growth factor \\
\hline
\end{tabular}




\begin{tabular}{|c|c|}
\hline Fig. & figure \\
\hline $5-\mathrm{FU}$ & fluorouracil \\
\hline FOLFIRINOX & folinic acid, fluorouracil, irinotecan and oxaliplatin \\
\hline $\mathrm{g}$ & gram \\
\hline Gly & glycine \\
\hline GM-CSF & granulocyte-macrophage colony-stimulating factor \\
\hline GEMM & genetically engineered mouse models \\
\hline $\mathrm{h}$ & hour \\
\hline HA & hyaluronic acid \\
\hline HBSS & hanks' balanced salt solution \\
\hline $\mathrm{HCl}$ & hydrochloric acid \\
\hline hENTs & human nucleoside transporters \\
\hline $\mathrm{H} \& \mathrm{E}$ & hematoxylin and eosin \\
\hline HEPES & 4-(2-hydroxyethyl)-1-piperazineethanesulfonic acid \\
\hline $\mathrm{Hh}$ & hedgehog \\
\hline His & histidine \\
\hline HRP & horse radish peroxidase \\
\hline HSP47 & heat shock protein 47 \\
\hline HSP90 & heat shock protein90 \\
\hline $\mathrm{H}_{2} \mathrm{O}_{2}$ & hydrogen peroxide \\
\hline $\mathrm{IgG}$ & immunoglobulin G \\
\hline IHC & immunohistochemistry \\
\hline IL6 & interleukin 6 \\
\hline IL8 & interleukin 8 \\
\hline ILK & integrin linked protein kinase \\
\hline $\mathrm{KC}$ & LSL-Kras ${ }^{\mathrm{G} 12 \mathrm{D}}$; Pdx or P48 Cre \\
\hline $\mathrm{kDa}$ & kilo Dalton \\
\hline KPC & LSL-Kras $^{\mathrm{G} 12 \mathrm{D}} ; \operatorname{Trp} 53^{172 \mathrm{H}} ; \mathrm{PdxCre}$ \\
\hline Lys & lysine \\
\hline LC-MS & liquid chromatography-mass spectrometry \\
\hline LSL & Lox-stop-lox \\
\hline $\mathrm{M}$ & mole \\
\hline MDSC & myeloid derived suppressor cells \\
\hline MEK1/2 & mitogen-activated protein kinase kinase $1 / 2$ \\
\hline MEM & minimum essential medium \\
\hline $\min$ & minute \\
\hline $\mathrm{ml}$ & millilitre \\
\hline $\mathrm{mM}$ & millimole \\
\hline MMP & matrix metalloproteinase \\
\hline M.O.M kit & mouse on mouse kit \\
\hline mRNA & messenger RNA \\
\hline mTORC1 & mammalian target of rapamycin complex 1 \\
\hline MTT & $\begin{array}{l}\text { 3-(4, 5-dimethylthiazol-2-yl)-2,5-diphenyl tetrazolium } \\
\text { bromide }\end{array}$ \\
\hline $\mathrm{NaOH}$ & sodium hydroxide \\
\hline NEAA & non-essential amino acid \\
\hline NF-KB & $\begin{array}{l}\text { nuclear factor kappa-light-chain-enhancer of activated B- } \\
\text { cells }\end{array}$ \\
\hline
\end{tabular}




\begin{tabular}{|l|l|}
\hline NFKBIA & NF-KB inhibitor $\alpha$ \\
\hline NT5c1A & 5'-Nucleotidase, Cytosolic IA \\
\hline NTP & nucleoside triphosphate \\
\hline PanIN & pancreatic intra epithelial neoplasm \\
\hline PAFs & PanIN associated fibroblasts \\
\hline PI3K & phosphoinositide 3 kinase \\
\hline PBS & phosphate Buffered Saline \\
\hline PBS-T & phosphate Buffered Saline withTween 20 \\
\hline PCR & polymerase chain reaction \\
\hline PDAC & pancreatic ductal adenocarcinoma \\
\hline PDGF & platelet derived growth factor \\
\hline pH & potential of hydrogen \\
\hline pM & pico mole \\
\hline PMSF & phenylmethanesulfonyl fluoride \\
\hline P/S & penicillin/Streptomycin \\
\hline PSCs & pancreatic stellate cells \\
\hline RNA & ribonucleic acid \\
\hline rpm & revolutions per minute \\
\hline RT & room temperature \\
\hline SBTI & soy bean trypsin inhibitor \\
\hline SDS & sodium dodecyl sulfate \\
\hline SDS-PAGE & SDS-polyacrylamide gel electrophoresis \\
\hline sec & second \\
\hline SMOC & secreted modular calcium binding protein \\
\hline SPARC & secreted protein acidic and rich in cysteine \\
\hline TAMs & tumor associated macrophages \\
\hline Taq & Thermus aquaticus \\
\hline TBE & tris-Borase-EDTA \\
\hline TBS & tris-buffered saline \\
\hline TBS-T & tris-buffered saline with Tween 20 \\
\hline TGF 3 & transforming growth factor beta \\
\hline TNF- $\alpha$ & tumor necrosis factor $\alpha$ \\
\hline TVA & tierversuchsantrag (animal experiment application) \\
\hline V & voltage \\
\hline VEGF & vascular endothelial growth factor \\
\hline vs & \\
\hline wt & zersus \\
\hline ZTE & \\
\hline
\end{tabular}




\begin{abstract}
Pancreatic ductal adenocarcinoma (PDAC) is one of the most aggressive solid tumors in humans. Median survival is around 12 months and is due to late diagnosis, early metastatic spread, and a high resistance towards available chemotherapeutic regimens. The desmoplastic reaction is a key feature of PDAC which contributes to disease progression and has also been reported to confer to chemoresistance and impaired drug delivery. Secreted protein acidic and rich in cysteine (SPARC) is an important matricellular protein that regulates collagen deposition and ECM remodeling. In human PDAC, SPARC is expressed by peritumoral fibroblasts and high expression is associated with a poor prognosis. In several other cancer entities, SPARC has been shown to play either tumor promoting or tumor suppressing roles. However, the functional role of SPARC in PDAC is unclear. In my thesis, I investigated the expression of SPARC and its role during tumor progression from preneoplastic lesions to frank carcinomas in genetically engineered mouse models (GEMMs) of PDAC. In order to achieve this, I generated SPARC ${ }^{\mathrm{wt}}, \mathrm{SPARC}^{-/-}, \mathrm{KC}^{-S P A R C}{ }^{\mathrm{wt}}$ and $\mathrm{KC}^{-S P A R C}{ }^{-/-}$mice with a global SPARC knock-out for in vivo studies. Furthermore, primary epithelial and fibroblast cell lines were derived from preneoplastic murine tissues and murine pancreas tumors for in vitro experiments.
\end{abstract}

The in vivo results showed that the development of the murine pancreas was unaffected by germ- line SPARC knock-out. Immunohistochemical and western blot analysis revealed that SPARC is not expressed in the normal pancreas with a marked increase of SPARC in activated fibroblasts during preneoplastic stages and tumor progression. However, loss of SPARC in $\mathrm{KC}-\mathrm{SPARC}^{-/-}$mice resulted in a significant reduction of intratumoral collagen deposition. Notably, SPARC and subsequent collagen depletion did not alter pancreatic intraepithelial neoplasia (PanIN) progression, tumor incidence or metastatic frequency to the liver. Both $\mathrm{KC}-\mathrm{SPARC} \mathrm{wt}^{\mathrm{w}}$ and $\mathrm{KC}^{-S P A R C} \mathrm{~S}^{-/-}$tumors exhibited similar tumor characteristic including proliferation, apoptosis and mean vessel density. Primary epithelial and fibroblast cell lines from both genotypes showed comparable morphology and proliferation rates. However, tumor bearing $\mathrm{KC}^{-S P A R C}{ }^{-/-}$mice lived significantly shorter than of KC-SPARC ${ }^{\mathrm{wt}}$ mice, a finding that was most likely due to more severe clinical complications such as ascites, diarrhea and bile duct obstruction in $\mathrm{KC}^{-S P A R C} \mathrm{C}^{-/}$mice. 
Interestingly, SPARC mediated collagen deposition did not impede the delivery and metabolism of gemcitabine in pre-neoplastic lesions and tumors as determined by LCMS/MS. Strikingly, the amount of gemcitabine increased from normal pancreas tissues to pancreatic tumors questioning the drug delivery hypothesis for gemcitabine in PDAC. 


\section{Introduction}

\subsection{Pancreatic ductal adenocarcinoma (PDAC)}

\subsubsection{Epidemiology}

Pancreatic ductal adenocarcinoma (PDAC) constitutes one of the most aggressive malignancies in humans (Carpelan-Holmstrom, 2005; Howlader et al., 2012) and accounts for 7\% of total cancers in both male and female (Carpelan-Holmstrom, 2005; Rahib et al., 2014; Schneider et al., 2005; Siegel et al., 2017). PDAC possesses one of the worst clinical outcomes of all solid tumors, and only about $6-8 \%$ of patients survive beyond 5 years. The median survival of untreated patients with advanced or metastatic disease is about $6-12$ months. Around $80 \%$ of patients are reported to have either locally advanced disease or distant metastases during initial diagnosis and cannot be operated anymore (Siegel et al., 2017).

In 2001, Parkin and colleagues have performed a study to examine the global cancer burden using multiple data sets from various sources including the World Health Organization (WHO), Disease surveillance points (DSP), GLOBCON 2000, International agency for Research on Cancer (IARC), Surveillance, Epidemiology and End Results (SEER) and EUROCARE-2 (Parkin et al., 2001). This study has shown that there were 216,000 new pancreatic cancer cases with an estimated death of 213,000 cases worldwide, while in Europe there were 60,139 newly reported cases with an estimated death of 64,801 cases (Parkin et al., 2001). In 2002, PDAC incidence in the United Kingdom was reported to be 7152 cases with the estimated death of 7250 cases (Ghaneh et al., 2008). More recently, the American Cancer Society reported 53,670 new cases in the US in 2017 with an estimated death of 43,090 cases (Siegel et al., 2017). Evidently, over the years the incidence is increasing while the mortality rate is unchanged (Lowenfels and Maisonneuve, 2004). Therefore, PDAC is the fourth common reason for cancer-related deaths worldwide, and it is expected to be the second leading cause of cancer-related deaths worldwide by 2030 .

There are various factors such as age, sex, ethnicity, smoking and alcoholic abuse, chronic pancreatitis, obesity, diabetes and Helicobacter pylori infections which have been associated with a slightly increased risk to develop PDAC (Maisonneuve and Lowenfels, 2010). In particular, tobacco and alcohol have been identified to accelerate 
the risk of PDAC development up to 2-fold (Iodice et al., 2008; Tramacere et al., 2010). PDAC occurs predominantly in the elderly population (Howlader et al., 2012) and is slightly more often diagnosed in males than in females (Siegel et al., 2017). One proposed explanation for this difference might be the protective role of female hormones against PDAC, though there is no concrete validation for this hypothesis yet (Wahi et al., 2009). Recent studies have also explored the direct correlation between obesity and PDAC risk (Arslan et al., 2010; Incio et al., 2016). The highest incidence is reported among the populations of industrialized/western countries, while the incidence seems to be relatively lower among Indian and Nigerian populations (Boyle et al., 1989).

PDAC is also associated with hereditary factors, in which germ line mutations in certain genes including BRCA1, BRCA2, APC, CDKN2A and PRSS1 result in PDAC development (Rizzato et al., 2013; Shi et al., 2009; Turati et al., 2013). Among these genes, BRCA2 was found to be the most frequently reported mutation to increase the risk of PDAC about 6-12\% (Murphy et al., 2002; Shi et al., 2009; Thompson and Easton, 2002).

\subsubsection{Current therapies of PDAC}

The treatment options for PDAC are extremely limited due to various reasons such as lack of early symptoms and diagnosis, high recurrence rate after surgery, and high resistance towards the existing chemotherapeutic regimens (Neesse et al., 2015). As mentioned earlier, only a small proportion of patients $(-20 \%)$ are eligible for surgery at the time of diagnosis (Neoptolemos et al., 2010). The prognosis of surgical patients is still poor due to local recurrence or distant metastases shortly after surgery. Therefore, patients who are eligible for surgery receive adjuvant therapy postoperatively. This has been shown to improve overall and disease free survival (Boeck et al., 2008; Stocken et al., 2005). Numerous clinical trials including CONKO-001, CONKO-005, JSAP-02, RTOG9704, European Study Group for Pancreatic cancer-1, 3, 4 (ESPAC-1, 3 and 4) have been performed over three decades to explore the optimal adjuvant therapy for PDAC (Kanji et al., 2018; Neoptolemos et al., 2010, 2017, Oettle et al., 2013, 2007; Sinn et al., 2017). Most of the above mentioned randomized trials studied the efficacy of gemcitabine alone or in combination with a second chemotherapeutic agent as adjuvant therapy following surgical resection. 
In 2010, the ESPAC-3 randomized and controlled trial was aimed to explore the therapeutic value of gemcitabine monotherapy compared to 5-fluorouracil (5-FU) plus folinic acid as an adjuvant therapy on a large number of patients who underwent surgical resection of PDAC. Results of this trial have shown that there was no significant difference in terms of overall survival and disease-free survival between the groups treated with gemcitabine alone (median survival- 23.6 months, progression free survival13.5 months) and the group treated with 5-FU plus folinic acid (median survival- 23.0 months, progression free survival-12.5 months). However, gemcitabine was tolerated well with less adverse side effects. Due to this advantage, gemcitabine has been considered as the standard adjuvant therapy (Neoptolemos et al., 2010). Recently, ESPAC-4 trial has aimed to study the clinical efficacy of gemcitabine and capecitabine combination compared to gemcitabine alone as an adjuvant therapy in surgically resected PDAC patients. This study has revealed that the combination had a significantly improved survival outcome compared to gemcitabine monotherapy while having a tolerable toxicity profile (overall median survival 28.8 months and 25.5 months respectively). Therefore, the combination of gemcitabine and capecitabine could be considered as the standard adjuvant therapy at present (Neoptolemos et al., 2017).

In case of locally advanced and metastatic PDAC palliative treatment represents the only approved treatment option at present. In the past, 5-FU was used to treat patients with advanced pancreatic cancer based on the study results of Cullinan and colleagues in 1985. In this study, the authors compared therapeutic efficacy of 5-FU versus the combination of 5-FU and doxorubicin versus the combination of 5-FU, doxorubicin and mitomycin and reported no significant difference in terms of survival and quality of life of the patients. Thus, 5-FU was considered the better option based on the most acceptable toxicity profile (Cullinan et al., 1985). In 1994, Casper and colleagues conducted a phase II trial using gemcitabine on patients with advanced PDAC with no prior treatment. This study reported an increased therapeutic efficacy of gemcitabine with a tolerable toxicity profile (Casper et al., 1994). Three years later, another randomized trial was conducted on a large cohort of patients (126 patients) to compare the clinical efficacy of gemcitabine versus standard 5-FU therapy. In this study, gemcitabine was shown to improve clinical benefit response (23.8\% and $4.8 \%$ respectively), and overall survival (5.65 and 4.41 months) compared to standard 5-FU treatment (Burris et al., 1997). Since then gemcitabine was used as a first line therapy for the locally advanced and metastatic 
PDAC for several decades. In 2011, Conroy and colleagues showed that FOLFIRINOX (folinic acid, fluorouracil, irinotecan and oxaliplatin) prolonged overall median survival of patients about 4.3 months compared to the gemcitabine monotherapy in a large phase III trial conducted in France (Conroy et al., 2011). Two years later, an international phase III trial reported a significantly prolonged overall survival of metastatic PDAC patients with gemcitabine and nab-paclitaxel combination treatment compared to gemcitabine monotherapy (Von Hoff et al., 2013). However, the above mentioned treatment regimens have more side effects than gemcitabine monotherapy, and not all patients are eligible based on age, performance and nutritional status.

\subsubsection{Initiation and progression}

Oncogenic Kras mutation occurs in almost 95\% of all human PDAC patients (Biankin et al., 2012; Jones et al., 2008; Kanda et al., 2012; Waddell et al., 2015; Witkiewicz et al., 2015). Kras mutations are also found in several other tumor entities including breast, endometrial, cervical, bladder, liver cancers and myeloid leukemia (Anderson et al., 1992; Bos et al., 1987; Downward, 2003; Schubbert et al., 2007). Kras ${ }^{\text {G12D }}$ is the most common point mutation in PDAC in which glycine is replaced by aspartic acid in the twelfth codon. Mutation results in constitutional activation of Kras activity which further leads to accelerated proliferation, differentiation and survival via downstream signalling cascades (Campbell et al., 1998). Oncogenic Kras stimulates cell proliferation by activating serine/threonine protein kinase B-Raf (BRAF) and thus by simultaneous activation of mitogen-activated protein kinase kinase1/2 (MEK1/2), extracellular signalregulated kinase (ERK) and ETS domain- containing protein Elk-1 (Elk-1). It mediates survival by concomitantly activating phosphoinositide 3 kinase (PI3K) - protein kinase $\mathrm{B}(\mathrm{AKT})$ and mammalian target of rapamycin complex 1 (mTORC1) pathway (Carpeño and Belda-iniesta, 2013). Oncogenic Kras is also known to stimulate cytokine production by activating serine/threonine protein kinase TBK1 (TBK1) which leads to nuclear translocation of nuclear factor kappa-light-chain-enhancer of activated B-cells (NF-KB) by releasing it from its inhibitory protein NFKBIA (NF-KB inhibitor $\alpha$ ) (Carpeño and Belda-iniesta, 2013). Nuclear translocation of NF-KB ultimately leads to transcription of various cytokine including IL-6 (Ancrile, 2009; Ancrile et al., 2007; Leslie et al., 2010), IL-8 (Sparmann and Bar-Sagi, 2004) and Granulocyte-macrophage colony-stimulating factor (GM-CSF) (Pylayeva-Gupta et al., 2012). However, Kras ${ }^{\mathrm{G} 12 \mathrm{D}}$ alone is not sufficient to initiate PDAC, and additional genetic events such as deletion or mutations 
in tumor suppressor genes P53, INK4a, SMAD4, PTEN, Mist and transforming growth factor $\beta$ (TGF- $\beta$ ) are required for progression to frank carcinoma (Guerra et al., 2007; Hahn et al., 1996; Hingorani et al., 2003; Morris et al., 2010). Moreover, inflammation induced by chronic pancreatitis in conjunction with Kras mutations also gives rise to pancreatic cancer as shown in genetically engineered mouse models (GEMMs) (Guerra et al., 2011).

Upon activation of oncogenic Kras signaling, acinar cells undergo a transition process called "acinar to ductal metaplasia" (ADM) in which the normal acinar cells lose the morphological and functional acinar characteristics (e.g. expression of amylase) and start to acquire the phenotypic and molecular properties of ductal cells. Subsequently, ADM further progress to pre-neoplastic lesions of PDAC - Pancreatic Intra epithelial Neoplasm (PanIN) (Morris et al., 2010). PanINs are by far the most common precursor to PDAC (Matthaei et al., 2011). The initial classification and nomenclature of PanINs as precursors for PDAC was established in 1999 (Klimstra and Longnecker, 1994).

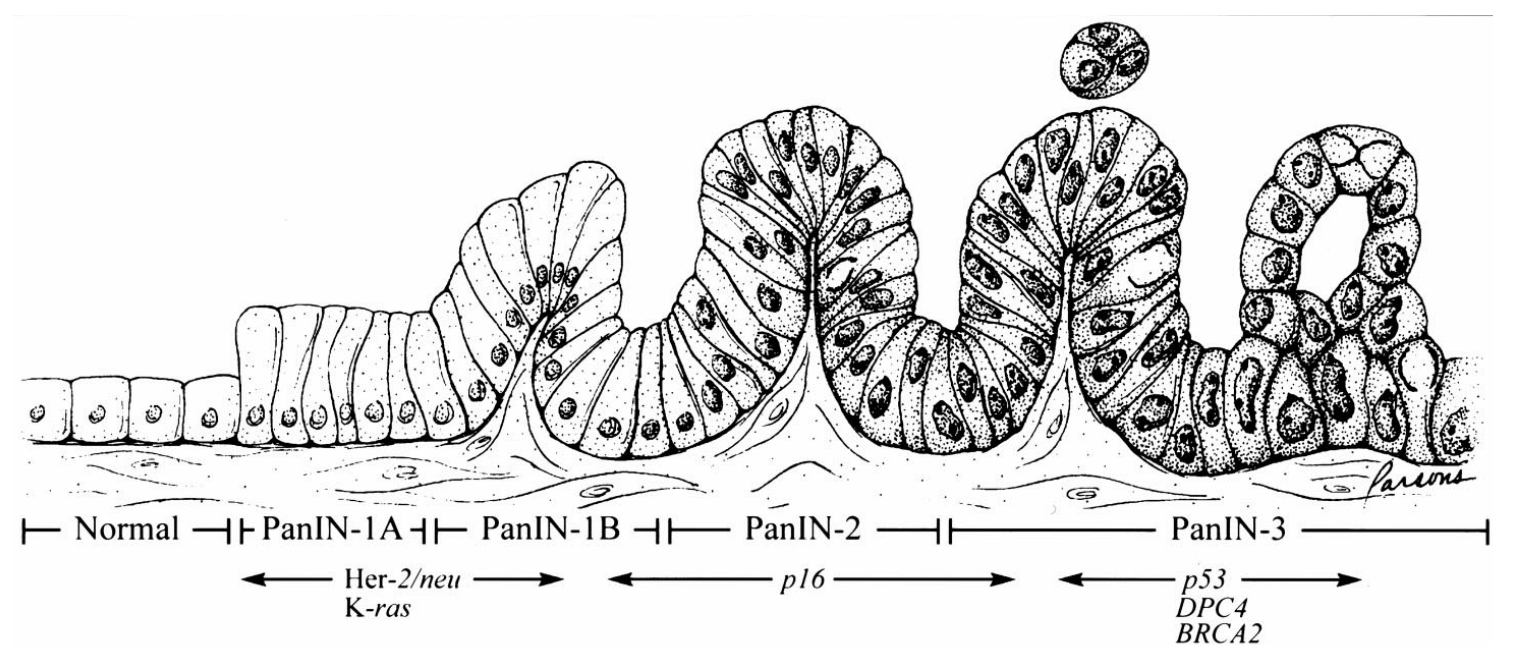

Figure 1: Initiation and progression of PDAC. Normal cuboidal (ductal) epithelial cells transform into tall columnar epithelium with basal nuclei which further attains pseudostratification and other cellular abnormalities. Based on the degree of atypia the pre-neoplastic lesions are classified from PanIN1-PanIN3. Throughout the progression multiple mutations are accumulated at various stages. (Figure adapted from Hruban et al., 2000).

During the progression of ADMs to PanINs, cells accumulate various cytological abnormalities including loss of polarity, nuclear crowding, mucinous cytoplasm and atypia. Based on the degree of cellular abnormalities, PanINs are further classified into three categories from PanIN1-3 (Hruban et al., 2004). PanINs cannot be detected in 
patients by currently available imaging methods, and up to now it is not clear at which frequency PanIN lesions eventually progress to PDAC.

\subsection{Significance of stroma in PDAC}

Notably, pancreatic stellate cells (PSCs) are already activated during early PanIN progression resulting in a pronounced accumulation of extracellular matrix components such as collagen and hyaluronic acid (Neesse et al., 2011, 2015).

Invasive PDAC harbors a pronounced desmoplastic reaction, and the tumor stroma comprises more than $90 \%$ of the entire tumor mass. The acellular compartment of stroma is made up of dense extracellular matrix components and various secreted growth factors. The cellular compartment of the stroma is comprised of abundant infiltrating inflammatory cells, activated fibroblasts, and altered endothelial cells (Chu et al., 2007; Neesse et al., 2011). Conflicting results have been published regarding the role of the tumor stroma in PDAC. Though different components of stroma have been shown to confer progression, invasion and chemoresistance of PDAC, there are few studies suggesting that stroma plays a tumor-suppressive role in PDAC (Chu et al., 2007; Ellenrieder et al., 2000; Hessmann et al., 2018; Hidalgo and Eckhardt, 2001; McclearyWheeler et al., 2012; Rhim et al., 2014). Recently, it was attempted to classify PDAC into subtypes based on stromal phenotypes (Collisson et al., 2011; Knudsen et al., 2017; Moffitt et al., 2015). Moffitt and colleagues have classified PDAC into basal (no stroma), normal and activated stromal subtypes and further assessed the survival of the respective subtypes using high throughput gene expression microarray analysis. In this study, the normal and activated stromal subtypes were differentiated by the expression of specific markers. The normal stroma expressed markers such as $\alpha$ - Smooth Muscle Actin $(\alpha-$ SMA), vimentin and desmin while the activated stroma was shown to express markers including secreted protein acidic and rich in cysteine (SPARC), chemokine (C-C motif) ligand 13, 18 (CCL13 and 18), gelatinase B and stromelysin 3. Additionally, there was a significant survival difference reported between these two stromal subtypes, in which the normal stromal subtype had a median survival of 24 months while the activated stromal subtype had a median survival of only 15 months (Moffitt et al., 2015).

\subsubsection{Acellular compartment of stroma}

The abundant extra cellular matrix (ECM) is composed of various soluble and nonsoluble factors. The most abundant non-soluble constituents of the ECM are collagen 
(type I, III and IV), laminin, fibronectin and hyaluronic acid (Thompson et al., 2010). Besides these solid components there are substantial number of soluble secreted factors such as connective tissue growth factor (CTGF), fibroblast growth factor (FGF), vascular endothelial growth factor (VEGF), platelet derived growth factor (PDGF) and TGF- $\beta$ (Apte et al., 2013; Feig et al., 2012; Gress et al., 1998; Lohr et al., 2001; Neesse et al., 2015; Ozawa et al., 2001). Apart from the above mentioned growth factors certain secreted proteins, which are collectively known as modulators of tumor - stroma interaction, are also abundantly found in the stromal compartment such as SPARC, periostin, thrombospondin and tenascin C (Baril et al., 2007; Esposito et al., 2006; Infante et al., 2007; Kanno et al., 2008; Lohr et al., 2001; Mantoni et al., 2008). The matrix metalloproteinases (MMPs) are a family proteolytic enzymes involved in multiple physiological functions including embryo implantation, development, angiogenesis, wound healing and tissue regeneration (Jabłońska-Trypuć et al., 2016). Among the big family of MMPs, MMP-2, MMP-9 and MMP-11 are known to be the crucial members of ECM composition (Iacobuzio-Donahue et al., 2003; Määttä et al., 2000; Qian et al., 2001; Shek et al., 2002; Vonlaufen et al., 2008a). Initially, MMPs were not only reported to facilitate metastatic spread by degrading the ECM barrier but also contributed to carcinogenesis (Chambers and Matrisian, 1997). The inflammatory cytokines (interleukin-1 (IL-1), interleukin-6 (IL-6) and tumor necrosis factor $\alpha$ (TNF- $\alpha$ )) and various growth factors (TGF- $\beta$, FGF, EGF and PDGF) are reported to increase the expression of MMPs. Thus by secreting the above mentioned inflammatory growth factors, tumor cells influence stromal cells in a paracrine manner to secrete more MMPs (Noël et al., 2008).

\subsubsection{Cellular compartment of stroma}

During the development of PDAC, there are various cell populations which are either activated within the pancreas or recruited from distant sites. Among them, inflammatory cells, activated fibroblasts and PSCs, endothelial cells and neurons are pivotal elements (Ceyhan et al., 2008; Demir et al., 2015; Neesse et al., 2011).

Tumor associated macrophages (TAMs), myeloid derived suppressor cells (MDSC) and regulatory $\mathrm{T}$ cells are found to be the most abundantly recruited immune cell population in PDAC. The dynamics of immune cell recruitment from PanIN to PDAC has been investigated in GEMMs of PDAC and showed abundant immunosuppressive MDSC already during early PanIN stages (Clark et al., 2007). These immune populations 
suppress $\mathrm{T}$ cell proliferation and response and were also shown to play a crucial role in tumor invasion by promoting ECM remodelling (Condeelis and Pollard, 2006; Hao et al., 2012; Pollard, 2004).

Activated fibroblasts or cancer associated fibroblasts (CAFs) are one of the most prominent cell populations within the tumor stroma. The majority of activated fibroblasts originate from PSCs. The resident fibroblasts of the pancreas are a sparse population which accounts for only 4\% of total cells of the healthy pancreas (Apte et al., 1998; Bachem et al., 1998). PSCs are normally found in a quiescent state and are activated upon inflammatory or oncogenic stimuli from the pre- neoplastic or neoplastic cells. In turn, the activated PSCs produce abundant ECM and other growth factors to aid the neoplastic growth (Apte et al., 2004; Habisch et al., 2010; Kalluri and Zeisberg, 2006; Schäfer et al., 2012). Studies have reported that CAFs can also be derived from bone marrow (Direkze et al., 2004; Ishii et al., 2003). Additionally, tumor cells were also shown to mimic the phenotypic and molecular properties of activated fibroblasts in certain conditions (Kalluri and Zeisberg, 2006). Several studies have emerged that reveal the contribution of PSCs regarding ECM production, angiogenesis, invasion, metastasis and chemoresistance of PDAC (Erkan et al., 2009; Hessmann et al., 2018; Lohr et al., 2001; Schneiderhan et al., 2007).

\subsection{Genetically engineered mouse models of PDAC}

The development of GEMMs is a breakthrough in PDAC research. GEMMs are particularly useful for the investigation of the tumor stroma since tumorigenesis occurs within the normal pancreatic microenvironment. Compared to xenograft and orthotopic models, GEMMs are particularly useful to investigate the desmoplastic reaction and study the crosstalk between stromal- and tumor cells.

GEMMs are generated by genetic mutations in oncogenes and tumor suppressor genes that also occur in human PDAC. To this end, activating mutations of the Kras oncogene occurs most frequently in PDAC and is critical for tumor development. Therefore, GEMMs of PDAC are generated by introducing a Kras ${ }^{\mathrm{G} 12 \mathrm{D}}$ mutation that is flanked by lox-stop-lox (LSL) sites and excised by Cre-recombinases that are driven under a pancreas-specific promoter such as Pdx or Ptfla (p48) that are expressed early during embryogenesis. Additional inactivating mutations in various tumor suppressor genes 
such as p53, SMAD4 or p16 can be combined to accelerate tumorigenesis (Aguirre et al., 2003; Ahmed et al., 2017; Bardeesy et al., 2006; Ijichi et al., 2006; Tuveson et al., 2006).

The LSL-Kras ${ }^{\mathrm{G} 12 \mathrm{D}}$; Pdx or P48 Cre (KC) model was initially established by Hingorani et al., 2003 (Hingorani et al., 2003; Jackson et al., 2001). The $\mathrm{KC}$ model is considered as a slow progression model as mice develop ADM and PanIN lesions over the course of several months. These PanIN lesions highly resemble the precursor lesions found in the patients. Around $60 \%$ of mice develop invasive carcinoma between 10-15 months of age. Therefore, the KC model is best suited to study the disease initiation and progression.

The LSL-Kras ${ }^{\mathrm{G} 12 \mathrm{D}}$; $\operatorname{Trp} 53^{172 \mathrm{H}}$;PdxCre (KPC) harbors an additional inactivating mutation of the p53 tumor suppressor gene that dramatically accelerates pancreatic tumorigenesis. Mice develop invasive pancreatic tumors between 3-6 months of age, and median survival is about 150 days (Hingorani et al 2005). Notably, KPC mice develop the full spectrum of PDAC including desmoplastic, locally invasive carcinoma, bile duct obstruction, ascites, cachexia and liver and lung metastasis. Therefore, the KPC model is appropriate to test response to novel therapies in preclinical studies and is currently the most frequently used GEMM of PDAC.

\subsection{Stromal targeting in PDAC}

Many preclinical studies and clinical trials have attempted to target different components and pathways of the tumor stroma as a therapeutic option. Though these anti-stromal approaches have shown promising results at the experimental level, they all failed in clinical trials (Bramhall et al., 2001, 2002), and there is currently no approved antistromal treatment for PDAC patients. For instance, Hedgehog $(\mathrm{Hh})$ signalling is one of the central regulators of tumor-stroma crosstalk which is shown to contribute to desmoplasia in various tumor entities (Thayer et al., 2003). Following an inflammatory or oncogenic stimuli, pancreatic epithelial cells express Hedgehog ligands (Fendrich et al., 2008; Thayer et al., 2003). In PDAC, Hh signalling has been shown to work in a paracrine manner in which Hh-ligands released by epithelial tumor cells affect the surrounding stromal cells and thus mediates desmoplasia (Lauth et al., 2010; Lee et al., 2014; Yauch et al., 2008). In 2009, Olive and colleagues have successfully depleted stromal deposition in a GEMM of PDAC by inhibiting Hh-signalling using a pharmacological inhibitor (IPI-926). The authors reported that co-administration of IPI926 and gemcitabine led to a significant increase of intra- tumoral gemcitabine 
accumulation and increased vascular density, and thus reduced the tumor growth. In contrast, four years later Rhim and colleagues showed that genetic inhibition of sonic hedgehog in a GEMM resulted in accelerated tumor growth with more aggressive tumor biology including undifferentiated histology, increased vascularity and proliferation of the tumors (Rhim et al., 2014). The data by Rhim et al explain the clinical failure of Hhinhibitors such as IPI-929. In the same year, Özdemir et al. depleted $\alpha$-SMA positive myofibroblasts in a GEMM of PDAC. In line with Rhim et al, this preclinical study revealed increased tumor invasiveness and aggressiveness upon myofibroblast depletion. Furthermore, myofibroblast depleted tumor showed pronounced epithelial to mesenchymal transition (EMT) features and an increased amount of cancer stem cells. Additionally, the authors also showed a correlation between decreased myofibroblast content and reduced survival in the patients (Özdemir et al., 2014).

Hyaluronic acid (HA) is one of the major stromal components of PDAC which has been shown to be correlated with poor survival (Whatcott et al., 2015). In preclinical experiments, HA depletion by the hyaluronidase PEGPH20 was shown to increase drug delivery and therapeutic efficacy of gemcitabine (Jacobetz et al., 2013; Provenzano et al., 2012; Singha et al., 2015). In a phase II clinical trial (HALO 202) PEGPH20 was combined with nab-paclitaxel/gemcitabine compared to the standard therapy nabpaclitaxel/gemcitabine to assess the therapeutic efficacy of HA depletion in PDAC patients (Hingorani et al., 2018). The results have shown that the highest benefit was achieved in PDAC patients with high intratumoral HA levels where an objective response rate of $45 \%$ vs $31 \%$, and overall survival of 11.5 vs 8.5 months was achieved compared to the control group (Hingorani et al., 2018). Currently, PDAC patients with high HAexpression are enrolled in a phase III trial to confirm the therapeutic potential of HAdepletion in PDAC patients (NCT02715804). 


\subsection{Secreted Protein Acidic Rich in Cysteine (SPARC)}

SPARC, also known as osteonectin or BM-40 is a $32 \mathrm{kDa}$, albumin and calcium binding matricellular protein (Sage et al., 1984), and belongs to a family of eight proteins (Bradshaw, 2012). Secreted modular calcium binding protein (SMOC) 1 and 2 (Vannahme et al., 2002, 2003), hevin (SPARC like 1) (Hambrock et al., 2003), testican 1, 2 and 3 (commonly known as SPOCK) (Alliel et al., 1993; Charbonnier et al., 1998; Schnepp et al., 2005; Vannahme et al., 1999) and folistatin like protein 1 (Hambrock et al., 2004) are the other members of the family.

Originally, SPARC has been identified as a bone specific protein which is reported to aid in mineralization of collagen (Termine et al., 1981). Unlike the typical structural proteins of the ECM (collagen, fibronectin and laminin), SPARC plays a non-structural role by serving as a mediator of cell-matix interaction (Brekken and Sage, 2000; Lane and Sage, 1994).

\subsubsection{Structure and biochemistry}

Human SPARC protein is encoded by a single copy gene located on chromosome 5q33.1. SPARC protein consists of three modular domains which are the acidic $\mathrm{NH}_{2}$-terminal domain (amino acid (aa) 1-52), the follistatin like domain (aa 53-137), and the Cterminal calcium binding domain (aa 138-286) (Hohenester et al., 1996; Lane and Sage, 1994; Martinek et al., 2002).

The $\mathrm{NH}_{2}$-terminal domain (domain I) includes a 17 aa signal peptide rich in aspartic and glutamic acid residues. Domain I of SPARC has been shown to bind Calcium ${ }^{2+}$ ions with low affinity and also to interact with hydroxyapatite (bone mineral) (Maurer et al., 1992; Romberg et al., 1985). Depending on the availability of $\mathrm{Ca}^{2+}$, this domain has been shown to bind a maximum of $8 \mathrm{Ca}^{2+}$ ions (Maurer and Hohenester, 1997). However, functions of the domain I are not completely calcium dependant (Lane et al., 1992). Specific sequences of domain I distinguish SPARC from other members of the SPARC like protein family.

Domain II of SPARC is rich in cysteine and is named as follistatin-like (FS) domain due its high homology with follistatin (a peptide which inhibits the secretion of follicle stimulating hormone) (Esch et al., 1987; Patthy, 1991). However, SPARC does not mimic the inhibitory function of follistatin against avtivin-A (Maurer et al., 1992). A synthetic peptide derived from follistatin-like domain (domain II) termed as peptide 2.1 
(aa 55-74) has exhibited an inhibitory effect on cell cycle progression similar to original SPARC protein in specific cell type such as bovine aortic endothelial cells (Funk and Sage, 1991). Another synthetic peptide derived from domain II (peptide 2.3-aa 113-130) has exhibited copper binding property due to the presence of Gly-His-Lys (GHL) residues (Funk and Sage, 1993; Lane T. F and Sage Helene., 1994; Vernon and Sage, 1989). Peptide 2.3 has been also shown to promote proliferation (in fibroblasts), angiogenesis and wound healing (Pickart and Lovejoy, 1987; Pickart et al., 1980).

The extracellular domain (EC domain or domain III) is highly conserved in SPARC of different species and among other members of the SPARC protein family. The EC domain of SPARC possesses the binding site for collagen type I and IV, and the two EFhands of the EC domain exhibit high affinity to calcium (Engel et al., 1987; Hohenester et al., 1997). Peptide 4.2 belongs to domain III (aa 254-273) and has been reported to impede the proliferation of endothelial cells (Kupprion et al., 1998; Motamed and Sage, 1998). The structure and domains of SPARC are depicted in Figure 2.
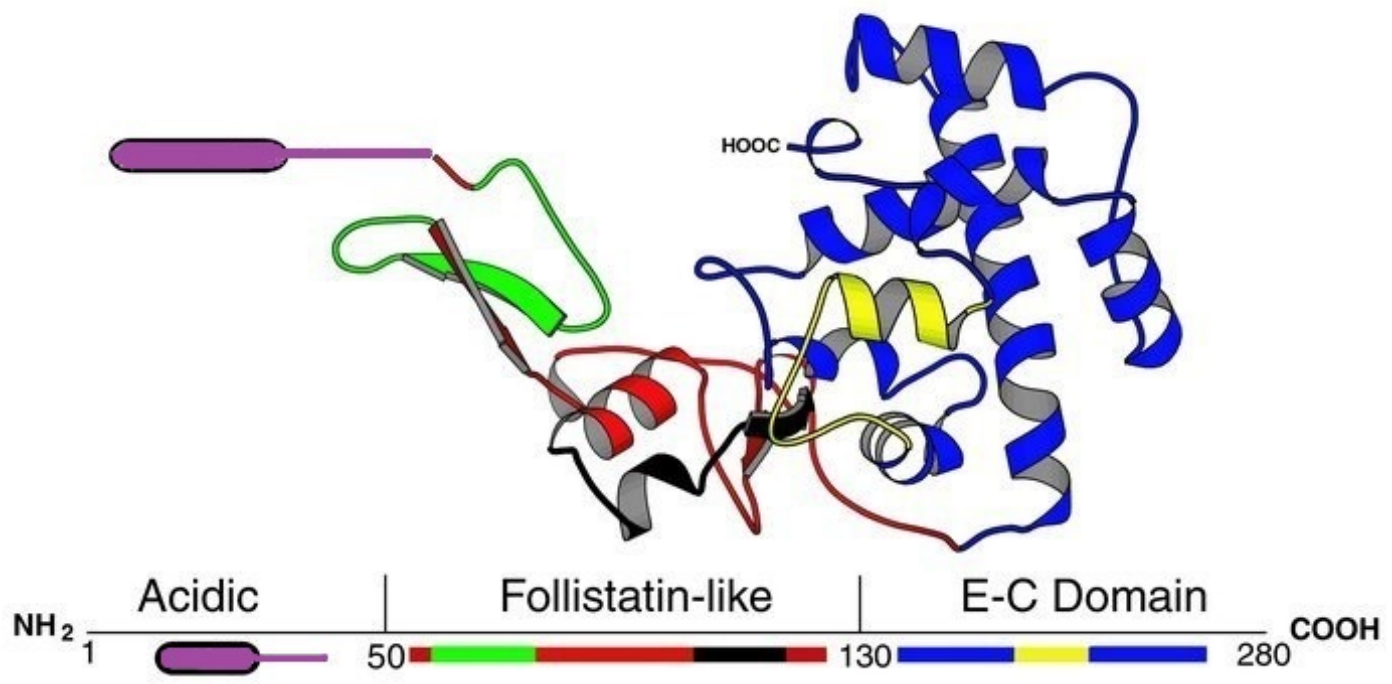

Figure 2: Structure of SPARC protein. Three domains of the SPARC protein, $\mathrm{NH}_{2}$ domain I, follistatin like domain and the extracellular $\mathrm{Ca}^{2+}$ binding domain are shown in purple, red and blue,respectively.Peptide2.1, peptide2.3 and peptide 4.2 are shown in green, black and yellow, respectively. Picture adapted from Brekken and Sage, 2000.

There are certain domain and tissue specific post translational modifications such as glycosylation (Asn 99-domain II), crosslinking between transglutaminases or disulfide crosslinking between SPARC that give rise to structural heterogeneity of SPARC. 
The heterogeneity in the protein structure allows SPARC to bind a wide range of proteins including different collagen subtypes (Kelm and Mann, 1991; Zhou et al., 1998). Additionally, certain structural similarities between SPARC family proteins explain their similar physiological roles (Bornstein, 1995).

\subsubsection{SPARC and ECM}

SPARC is a modulator of ECM whose expression is reported to be associated with increased ECM deposition (Framson and Sage, 2004). SPARC binds to various structural and non-structural proteins of the ECM including collagen (type I, II, III, IV, V and VIII) (Maurer et al., 1992; Sage et al., 1989; Termine et al., 1981), vitronectin (Rosenblatt et al., 1997) and thrombospondin (Clezardin et al., 1988). Collagen is one of the predominant components of the ECM which plays a significant role during embryonic development. The proper assembly and folding of collagen is mandatory for the biophysical and biochemical functions maintaining the homeostasis of the basal lamina and various connective tissues (Martinek et al., 2006). There are various molecular chaperons that are involved in the folding, assembling and maturation of collagen. Among them, heat-shock protein 47 (HSP47) is one of the best studied molecular chaperons of collagen whose homozygous deletion results in prenatal lethality in mice (Poschl, 2004). SPARC is a molecular chaperon of collagen due to its ability to bind to the triple helical domains of collagen. In concert with HSP47, SPARC mediates stability of collagen before its secretion from the endoplasmic reticulum (Martinek et al., 2006; Wang et al., 2005). Consistently, Fisher et al., have shown co-expression of HSP47 and SPARC during high collagen synthesis. However, unlike HSP47, homozygous SPARC knock out is not lethal in mice. It has been reported that the type I collagen fibrils of SPARC null mice are smaller than that of SPARC wildtype mice fibrils confirming the participation of SPARC in collagen folding and maturation (Bradshaw et al., 2003). Unlike HSP47, SPARC is thought to be still bound to procollagen during post endoplasmic reticulum (ER) events thus preventing side-to-side aggregation of procollagen (Martinek et al., 2006). The contribution of SPARC in collagen folding, fibrillogenesis and deposition is shown in Fig. 3. 


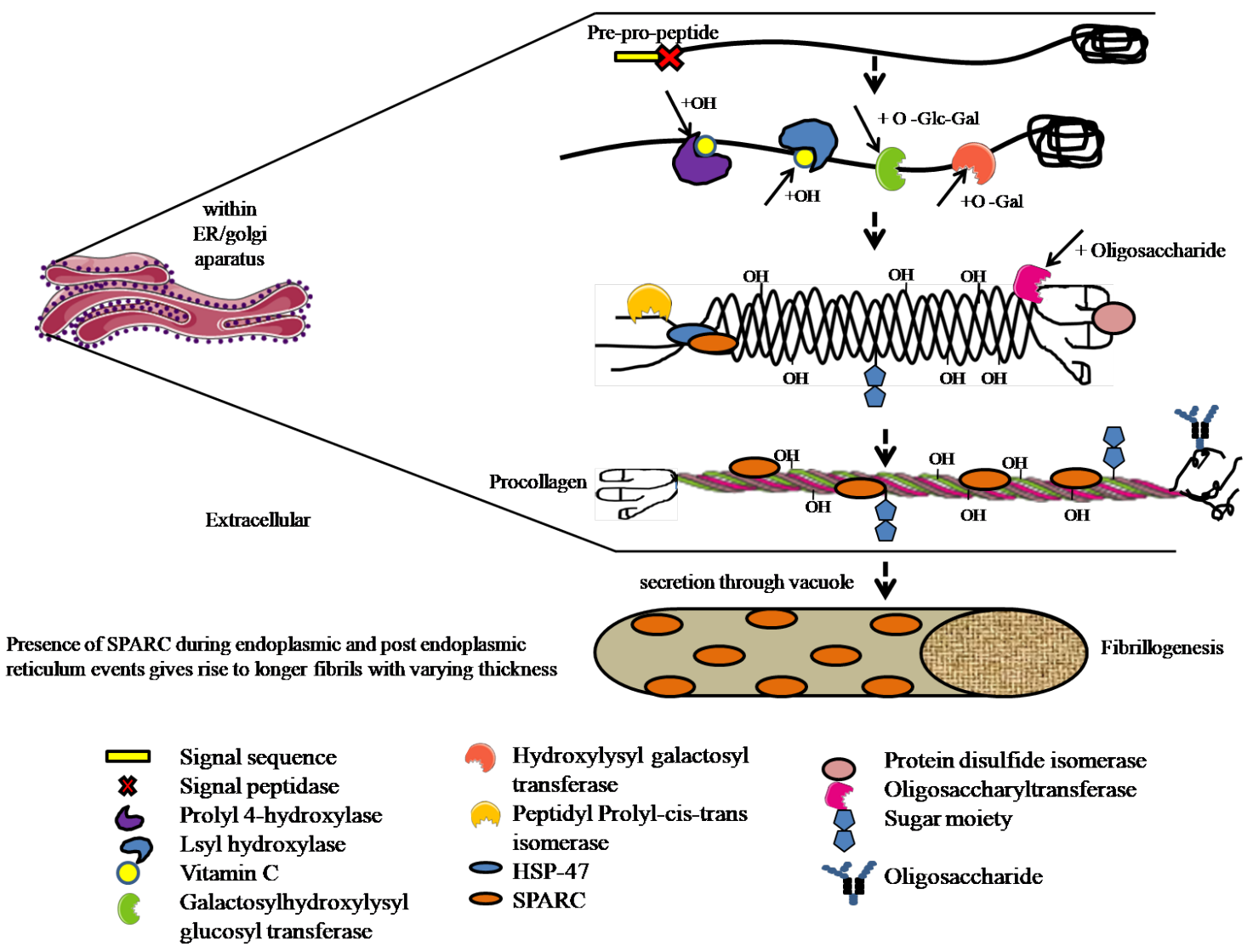

Figure 3: A model showing the contribution of SPARC during endoplasmic and post-endoplasmic events in collagen fibrillogenesis. SPARC mediates proper folding of collagen together with HSP47, a known molecular chaperon of collagen. Unlike HSP47, SPARC is still bound to procollagen upon secretion and thus prevents self-aggregation of premature collagen which ultimately leads to the formation of mature and thicker collagen fibrils.

Studies have also discussed various other possibilities by which SPARC maintains collagen homeostasis by interacting with intracellular components like Integrin linked protein kinase (ILK) or by regulating the activity matrix metalloproteinases and certain growth factors (Martinek et al., 2006). Studies have also shown the significance of fibronectin assembly at the cell surface in collagen fibrillogenesis (Robinson et al., 2004). There are certain ligands and receptors such as $\alpha 2 \beta 1$ integrin ligand, fibronectin and discoidin domain receptor 2 (DDR2) which are reported to share their binding site on collagen (aa 600-800) with SPARC (Carafoli et al., 2009; Ingham et al., 2002; Wang et al., 2005; Xu et al., 2000). In 2005, Barker and colleagues have shown a direct interaction between SPARC and ILK and they further claimed that SPARC modulates many signaling pathways including TGF- $\beta$ by acting as a counter-adhesive molecule. Additionally, SPARC has also been shown to regulate collagen fibrillogenesis by 
regulating the activity of decorin, an important proteoglycan involved in collagen fibrillogenesis (Barker et al., 2005).

\subsubsection{SPARC and growth factor signalling}

SPARC has been reported to interact with various secreted growth factors such as PDGF, VEGF, TGF- $\beta$ and basic fibroblast growth factor (bFGF) (Francki et al., 1999b; Hasselaar and Sage, 1992; Kupprion et al., 1998; Raines et al., 1992). Thus, SPARC mediates a wide range of physiological functions by altering ECM organization and by interacting with intracellular growth factor signalling (Funk and Sage, 1991; Tremble et al., 1993; Yan and Sage., 1999). SPARC has been shown to bind PDGF and VEGF directly and thus preventing the ligand binding which further results in inactivation of the respective receptors and receptor mediated cellular functions. SPARC reduced the PDGF and VEGF mediated proliferation in human arterial vascular smooth muscle cells and microvascular endothelial cells, respectively (Kupprion et al., 1998; Raines et al., 1992). SPARC has been also described to regulate the TGF- $B$ signalling pathway (Francki et al., 1999a; Schiemann et al., 2003). There is a mutual regulatory feedback loop that was identified between SPARC and TGF- $\beta$ due to the ability to regulate each other's expression (Bassuk et al., 2000; WRANA et al., 1991). Unlike the direct interaction of SPARC with other growth factors, SPARC does not directly bind bEGF. However, an inhibitory effect against bFGF mediated migration of endothelial cell was reported (Hasselaar and Sage, 1992).

\subsubsection{SPARC in cancer}

Overexpression or loss of SPARC has been reported in many human tumor entities including breast, colon, oesophageal cancers and melanoma (Sato et al., 2003). SPARC seems to play pro-tumorigenic role in certain cancers such as non-small cell lung cancer, melanoma, glioblastoma while it has been ascribed a tumor suppressive role in other cancers such as colorectal, ovarian, acute myelogenous leukemia and prostate cancers (Chlenski et al., 2002; Dhanesuan et al., 2002; Fernanda Ledda et al., 1997; Koblinski et al., 2005; Koukourakis et al., 2003; Said et al., 2013; Shin et al., 2013; Suzuki et al., 2005; Yiu et al., 2001). 


\subsubsection{SPARC in PDAC}

In PDAC, SPARC is highly expressed by peritumoral fibroblasts, while there is merely any expression in the tumor cell itself. Furthermore, high expression of SPARC in the stromal compartment was correlated with poor prognosis in PDAC patients (Infante et al., 2007; Mantoni et al., 2008). The loss of SPARC in epithelial tumor cells is associated with hypermethylation of the SPARC promoter. In particular, hypermethylation of the CPG-2 region is closely associated with pathophysiological conditions of the pancreas such as pancreatitis and PDAC (Gao et al., 2010; Sato et al., 2003). Different in vitro and in vivo studies have reported both tumor suppressive and tumor promoting roles of SPARC in PDAC. For instance, the tumor suppressive role of SPARC was shown by the study of Chen and colleagues, in which the authors reported a reduced growth and migration of PDAC cell lines in the presence of exogenous SPARC. Furthermore, a correlation between endogenous SPARC expression and reduced tumor aggressiveness was reported in a xenograft mouse model (Chen et al., 2010). In 2004, a study performed by Puolakkainen et al. revealed the complex nature of SPARC in tumorigenesis. Using subcutaneously implanted tumors in SPARC deficient mice, the authors reported an accelerated tumor growth which was associated with reduced ECM deposition and reduced apoptosis of tumor cells (Puolakkainen et al., 2004).

Contradicting the above studies, SPARC also has been shown to play a protumorigenic role. Guweidhi et al., provided evidence that upon exogenous SPARC treatment Colo357 (low endogenous SPARC expression) cells exhibited an increased invasiveness in vitro. Similarly, inhibition of endogenous SPARC in PANC-1 (high endogenous SPARC expression) resulted in diminished invasion. Moreover, exogeneous SPARC treatment resulted in increased invasiveness of PDAC cell lines with parallel overexpression of MMP-2 (Guweidhi et al., 2005). Another study has revealed a negative correlation between stromal SPARC expression and overall survival of PDAC patients (Mantoni et al., 2008).

Importantly, the role of peritumoral SPARC has not been assessed in appropriate experimental models that recapitulate the pronounced tumor microenvironment. To this end, GEMMs of PDAC seem to be particularly suited to investigate the role of stromal derived SPARC in PDAC. 


\subsection{Aim of the study}

Abundant collagen and HA deposition is a hallmark feature of the tumor microenvironment in PDAC. Several preclinical studies suggest that pharmacological depletion of collagen (e.g. by losartan) and HA (by PEGPH20) relieves vessel compression thus improving drug delivery and therapeutic response (Chauhan et al., 2013; Diop-Frimpong et al., 2011; Jacobetz et al., 2013; Provenzano et al., 2012). In contrast, recent evidence in several GEMMs casted serious doubt on the stromal depletion strategy since preclinical results have shown that pancreatic tumors become more aggressive, invasive and undifferentiated upon genetic or pharmacological inhibition of pro-stromal signaling pathways such as the SHH-pathway (Özdemir et al., 2014; Rhim et al., 2014). Therefore, stromal reprogramming rather depletion has become a novel approach to attempt to normalize tumor stroma rather than deplete it (Neesse et al., 2013; Sherman et al., 2014). To achieve this, a detailed knowledge of pro-tumorigenic and tumor-suppressive properties of the tumor stroma is required. Indeed, detailed molecular studies have identified first features of CAFs that distinguish between tumor promoting and tumor suppressive functions (Öhlund et al., 2017).

In my thesis, I aim to examine the role of SPARC in PDAC. SPARC is overexpressed in human and murine CAFs in the tumor stroma of PDAC, whereas it is hardly expressed in preneoplastic and neoplastic cells. A recent study by Moffitt et al. provided first evidence that SPARC is an important marker of the activated tumor stroma in PDAC patients that is associated with a significantly shortened survival (Moffitt et al., 2015). However, it is not known whether and how peritumoral SPARC contributes to disease progression in PDAC. Furthermore, as SPARC is part of the activated tumor stroma and has been implicated in collagen deposition and remodelling, I hypothesize that genetic ablation of SPARC in a GEMM of PDAC may reprogram the tumor stroma, slowing down tumor progression and increasing drug accumulation and efficacy by collagen remodelling.

To address this hypothesis, I crossed the KC model with a germ-line SPARC knock-out mouse. This mouse model allowed me to address the impact of SPARC during different stages of PanIN and tumor development, as well as metastasis formation. Furthermore, this model allows to conduct preclinical assays to investigate the role of SPARC dependent collagen remodelling on drug delivery and efficacy. 


\section{Materials}

\subsection{Technical instruments}

\begin{tabular}{|c|c|}
\hline Equipment & Company \\
\hline $\begin{array}{l}\text { Agarose gel electrophoresis } \\
\text { chamber-Model 40-0911 }\end{array}$ & $\begin{array}{l}\text { Peqlab Biotechnologie GmbH, Erlangen, } \\
\text { Germany }\end{array}$ \\
\hline Arium ${ }^{\circledR}$ pro ultrapure water system & Sartorius, Göttingen, Germany \\
\hline Aspirator with trap flask & Grant Instruments Ltd, Cambs, England \\
\hline Autoclave & $\begin{array}{l}\text { TecnomaraIntegraBiosciences, Deutschland } \\
\text { GmbH }\end{array}$ \\
\hline Autoclave (heat sterilization) & SHP Steritechnik AG, Haldensleben, Germany \\
\hline Centrifuge (Universal 320R) & Hettich lab technology, Tuttlingen, Germany \\
\hline $\begin{array}{l}\text { Centrifuge - Perfect spin } 24 \text { plus } \\
\text { (Prism R) }\end{array}$ & $\begin{array}{l}\text { Peqlab Biotechnologie GmbH, Erlangen, } \\
\text { Germany }\end{array}$ \\
\hline $\begin{array}{l}\text { Centrifuge (HeraeusMultifuge } \\
\text { X1R) }\end{array}$ & Thermo Fisher scientific, Waltham, USA \\
\hline Cellometer $\AA$ Auto 1000 cell counter & Nexcelom Bioscience, Lawrence, MA \\
\hline Class II safety cabinet (S2020 -1.2) & Thermo Fisher scientific, Waltham, USA \\
\hline Cold plate (Histocore Arcadia c) & Leica Biosystem, Wetzlar, Germany \\
\hline Flex cycler block & Analytikjena Germany \\
\hline $\begin{array}{l}\text { Molecular } \\
\text { Imager }{ }^{\circledR} \text { Chemidoc }{ }^{\mathrm{TM}} \text { XRS System }\end{array}$ & Bio-rad Hercules USA \\
\hline Heating plate & Leica Biosystem, Wetzlar, Germany \\
\hline HERAcell 240i $\mathrm{CO}_{2}$ incubator & Thermo Scientific, Waltham, USA \\
\hline Ice flaker (AF80) & Scotsman, Edinburgh, UK \\
\hline INTAS- ECL Chemocam imager & $\begin{array}{l}\text { INTAS Science Imaging Instruments } \mathrm{GmbH} \text {, } \\
\text { Göttingen, Germany }\end{array}$ \\
\hline $\begin{array}{l}\text { Inverted live cell microscope DMi8 } \\
\text { automated }\end{array}$ & Leica Microsystems GmbH, Wetzlar, Germany \\
\hline Inverted microscope (CKX53SF) & Olympus, Tokyo, Japan \\
\hline Light microscope "BX43" & Olympus, Tokyo, Japan \\
\hline Magnetic stirrer (RH B S000) & IKA ${ }^{\circledR}$ Laboratory equipment, Germany \\
\hline Microplate Luminometer "LUMO" & $\begin{array}{l}\text { Autobiolabtec Instruments Co.,Ltd, } \\
\text { Zhengzhou, China }\end{array}$ \\
\hline Microplate reader "PHOmo" & $\begin{array}{l}\text { Autobiolabtec Instruments Co.,Ltd, } \\
\text { Zhengzhou, China }\end{array}$ \\
\hline Microwave (NN-E209W) & Panasonic; Japan \\
\hline Microtome (Leica RM2265) & Leica Biosystems, Wetzlar, Germany \\
\hline Mini centrifuge (SPROUT ${ }^{\mathrm{TM}}$ ) & HealthrowScientific ${ }^{\mathbb{R}}$ LLC, Illinois, USA \\
\hline Mini gel tank (A25977) & $\begin{array}{l}\text { Invitrogen Thermo Fisher scientific, Waltham, } \\
\text { USA }\end{array}$ \\
\hline Mini-water bath & Bodo Schmidt GmbH, Göttingen, Germany \\
\hline $\begin{array}{l}\text { Multi-functional orbital shaker } \\
\text { (PSu-20i) }\end{array}$ & Grant Instruments Ltd., Cambs, England \\
\hline Multipipette ${ }^{\circledR}$ plus & Eppendorf AG Hamburg, Germany \\
\hline $\begin{array}{l}\text { Paraffin Tissue embedder } \\
\text { (EG1150H) }\end{array}$ & Leica Biosystems, Wetzlar, Germany \\
\hline
\end{tabular}




\begin{tabular}{|c|c|}
\hline pH meter (FiveEasy ${ }^{\mathrm{TM}}$ Plus FEP20) & $\begin{array}{l}\text { Mettler-Toledo AG, Schwerzenbach, } \\
\text { Switzerland }\end{array}$ \\
\hline Pipetus ${ }^{\circledR}$ Akku & Hischmann, Laborgerate, Eberstadt, Germany \\
\hline Pipetor - pipetboyacu 2 & Integra Biosciences, Zizers, Switzerland \\
\hline Pipettes Research series & Eppendorf AG Hamburg, Germany \\
\hline PowerPac ${ }^{\mathrm{TM}} \mathrm{HC}$ & Bio-rad Hercules USA \\
\hline Refrigerator $4^{\circ} \mathrm{C}$ (Comfort) & Liebherr, Bulle, Switzerland \\
\hline $\begin{array}{l}\text { Shandon coverplate }{ }^{\mathrm{TM}} \text { (cadenza } \\
\text { system) }\end{array}$ & Thermo Scientific, Waltham, USA \\
\hline $\begin{array}{l}\text { Sequenza }{ }^{\mathrm{TM}} \text { slide rack(cadenza } \\
\text { system) }\end{array}$ & TED PELLA,INC, Redding, CA \\
\hline T100 ${ }^{\mathrm{TM}}$ Thermal cycler & Bio-rad Hercules USA \\
\hline Thermo mixer compact & Eppendorf AG Hamburg, Germany \\
\hline Universal oven UF260 & $\begin{array}{l}\text { MemmertGmbH+Co. KG, Schwabach, } \\
\text { Germany }\end{array}$ \\
\hline Universal oven UF55 pa & $\begin{array}{l}\text { MemmertGmbH+Co. KG, Schwabach, } \\
\text { Germany }\end{array}$ \\
\hline Vacuum pump & $\begin{array}{l}\text { VacuubrandGmbH+Co. KG, Wertheim, } \\
\text { Germany }\end{array}$ \\
\hline Vortex (MS1) & IKA $^{\circledR}$ works do BrasilLtda, Taquara, Brazil \\
\hline Vortex (REAX1) & $\begin{array}{l}\text { HeidolphInstruments GmbH \& Co. KG, } \\
\text { Schwabach, Germany }\end{array}$ \\
\hline Waterbath (WNB14) & $\begin{array}{l}\text { MemmertGmbH+Co. KG, Schwabach, } \\
\text { Germany }\end{array}$ \\
\hline Weighing balance & Sartorius AG, Göttingen, Germany \\
\hline$-20^{\circ} \mathrm{C}$ freezer & Liebherr, Bulle, Switzerland \\
\hline $\begin{array}{l}-80^{\circ} \mathrm{C} \text { Ultra low temperature freezer } \\
(\mathrm{MDF}-\mathrm{U} 54 \mathrm{~V})\end{array}$ & Sanyo electric Co.,Ltd Japan \\
\hline $\begin{array}{l}\text { Tissue dehydration machine } \\
\text { (automated) }\end{array}$ & Leica Biosystems, Wetzlar, Germany \\
\hline
\end{tabular}

\subsection{General consumables}

\begin{tabular}{|c|c|}
\hline Goods & Company \\
\hline Aluminium foil & $\begin{array}{l}\text { Carl Roth GmbH Co. KG, Karlsruhe, } \\
\text { Germany }\end{array}$ \\
\hline Centrifuge tube 15 and $50 \mathrm{ml}$ & Sarstedt, Nümbrecht, Germany \\
\hline Cell strainer- $100 \mu \mathrm{m}$ & Falcon, Durham, USA \\
\hline Cell scrapper & Sarstedt, Nümbrecht, Germany \\
\hline Cello meter counting chamber & Nexcelom Bioscience, Lawrence, MA \\
\hline Cryo pure vial & Sarstedt, Nümbrecht, Germany \\
\hline Combitips advanced (Multipipette tips) & $\begin{array}{l}\text { Starlab International GmbH, Hamburg, } \\
\text { Germany }\end{array}$ \\
\hline $\begin{array}{l}\text { Graduated sterile pipettes }(2,10,25 \text { and } \\
50 \mathrm{ml})\end{array}$ & Greiner bio-one, Frickenhausen, Germany \\
\hline Glass coverslips $(24 \times 32,24 \times 60)$ & $\begin{array}{l}\text { Menzel Gläser }{ }^{\circledR} \text {, Menzel GmbH+Co KG, } \\
\text { Braunschweig, Germany }\end{array}$ \\
\hline Micropipette filter tips & $\begin{array}{l}\text { Starlab International GmbH, Hamburg, } \\
\text { Germany }\end{array}$ \\
\hline
\end{tabular}




\begin{tabular}{|l|l|}
\hline Micropipette tips & $\begin{array}{l}\text { Starlab International GmbH, Hamburg, } \\
\text { Germany }\end{array}$ \\
\hline Microtome blade (S35) & $\begin{array}{l}\text { Feather safety Razer Co. Ltd, Osaka, } \\
\text { Japan }\end{array}$ \\
\hline Microtube (1.5 and 2ml) & Sarstedt, Nümbrecht, Germany \\
\hline Injection needle (Sterile) & B.Braun, Melsungen, Germany \\
\hline Nitrocellulose membrane & $\begin{array}{l}\text { GE Healthcare lifesciences, Marlborough, } \\
\text { USA }\end{array}$ \\
\hline Parafilm & Pechiney plastic packaging, Chicago, USA \\
\hline PCR tubes & Sarstedt, Nümbrecht, Germany \\
\hline Scalpel & $\begin{array}{l}\text { Feather safety Razer Co. Ltd, Osaka, } \\
\text { Japan }\end{array}$ \\
\hline Serological pipette (2ml) & Greiner bio-one, Frickenhausen, Germany \\
\hline Sponge Pad ForXCell IITM Blotting & Novex by Life technologies, Carlsbad, CA \\
\hline Superfrost glass slides (Histology) & Thermo Scientific, Waltham, USA \\
\hline Syringe (1ml) & BD Plastic, Madrid, Spain \\
\hline Syringe $(5,10,20 \mathrm{ml})$ & B.Braun, Melsungen, Germany \\
\hline Sterile syringe Filter $(0.2 \mu \mathrm{m})$ & $\begin{array}{l}\text { Labsolute, Th.Geyer ingredients GmbH \& } \\
\text { Co.KG, Höxter, Germany }\end{array}$ \\
\hline Tissue culture multiwell plates & Greiner bio-one, Frickenhausen, Germany \\
\hline Tissue culture flasks & Greiner bio-one, Frickenhausen, Germany \\
\hline Tissue culture dishes $(2 \mathrm{~cm}, 10 \mathrm{~cm})$ & Sarstedt, Nümbrecht, Germany \\
\hline Tissue cassette & Sanowa, Leimen, Germany \\
\hline
\end{tabular}

\subsection{Chemicals}

\subsubsection{General chemicals}

\begin{tabular}{|l|l|}
\hline \multicolumn{1}{|c|}{ Chemical } & \multicolumn{1}{c|}{ Company } \\
\hline Albumin standard & Thermo Scientific, Waltham, USA \\
\hline Acetic acid & Sigma-Aldrich, St. Louis, USA \\
\hline Agarose & $\begin{array}{l}\text { Biozym Scientific GmbH, Oldendorf, } \\
\text { Germany }\end{array}$ \\
\hline Aqua & B.Braun, Melsungen, Germany \\
\hline BSA & Serva, Heidelberg, Germany \\
\hline B-Mercaptoethanol & Merck, Darmstadt, Germany \\
\hline Boric acid & Merck, Darmstadt, Germany \\
\hline Calcium chloride & Applichem, Darmstadt, Germany \\
\hline Citric acid monohydrate & $\begin{array}{l}\text { Carl Roth GmbH Co. KG, Karlsruhe, } \\
\text { Germany }\end{array}$ \\
\hline EDTA & Acros organics, Geel, Belgium \\
\hline EGTA & Sigma-Aldrich, St. Louis, USA \\
\hline Ethanol & $\begin{array}{l}\text { ChemSolute } \\
\text { GmbH \& Th. Geyer ingredients }\end{array}$ \\
\hline Eosin & Sigma-Aldrich, St. Loüxter, Germany \\
\hline Formaldehyde (4\%) & Merck, Darmstadt, Germany \\
\hline HEPES & $\begin{array}{l}\text { Carl Roth GmbH Co. KG, Karlsruhe, } \\
\text { Germany }\end{array}$ \\
\hline
\end{tabular}




\begin{tabular}{|l|l|}
\hline Glycerol & $\begin{array}{l}\text { Carl Roth GmbH Co. KG, Karlsruhe, } \\
\text { Germany }\end{array}$ \\
\hline Hematoxylin & Sigma-Aldrich, St. Louis, USA \\
\hline $\mathrm{HCl}$ & $\begin{array}{l}\text { Carl Roth GmbH Co. KG, Karlsruhe, } \\
\text { Germany }\end{array}$ \\
\hline $\mathrm{H}_{2} \mathrm{O}_{2}$ & $\begin{array}{l}\text { Carl Roth GmbH Co. KG, Karlsruhe, } \\
\text { Germany }\end{array}$ \\
\hline Isofluran & $\begin{array}{l}\text { AbbVie Deutschland GmbH \& Co. } \\
\text { KG, Ludwigshafen, Germany }\end{array}$ \\
\hline Magnesium chloride & Applichem, Darmstadt, Germany \\
\hline Methanol & $\begin{array}{l}\text { Carl Roth GmbH Co. KG, Karlsruhe, } \\
\text { Germany }\end{array}$ \\
\hline Non-fat milk powder & $\begin{array}{l}\text { Carl Roth GmbH Co. KG, Karlsruhe, } \\
\text { Germany }\end{array}$ \\
\hline PBS (Dulbecco's) & Biochrom, Berlin, Germany \\
\hline PMSF & Sigma-Aldrich, St. Louis, USA \\
\hline Roticlear & $\begin{array}{l}\text { Carl Roth GmbH Co. KG, Karlsruhe, } \\
\text { Germany }\end{array}$ \\
\hline Rotimount & $\begin{array}{l}\text { Carl Roth GmbH Co. KG, Karlsruhe, } \\
\text { Germany }\end{array}$ \\
\hline Saline (0,9\% NaCl) & B.Braun, Melsungen, Germany \\
\hline Sodium chloride & Merck, Darmstadt, Germany \\
\hline Sodium citrate & Sigma-Aldrich, St. Louis, USA \\
\hline Sodium fluoride & Sigma-Aldrich, St. Louis, USA \\
\hline Sodium hydroxide & Acros organics, Geel, Belgium \\
\hline Sodium Orthovanadate & Sigma-Aldrich, St. Louis, USA \\
\hline Sodium pyrophosphate & Sigma-Aldrich, St. Louis, USA \\
\hline Sodium pyruvate & $\begin{array}{l}\text { Gibco }{ }^{\circledR} \text { Thermo scientific, Waltham, } \\
\text { USA }\end{array}$ \\
\hline Tris-HCl & $\begin{array}{l}\text { Carl Roth GmbH Co. KG, Karlsruhe, } \\
\text { Germany }\end{array}$ \\
\hline Tris-base & $\begin{array}{l}\text { Carl Roth GmbH Co. KG, Karlsruhe, } \\
\text { Germany }\end{array}$ \\
\hline Triton X-100 & Sigma-Aldrich, St. Louis, USA \\
\hline Tween-20 & Sigma-Aldrich, St. Louis, USA \\
\hline & \\
\hline
\end{tabular}

\subsubsection{Special chemicals and reagents}

\begin{tabular}{|c|c|}
\hline Chemical or reagent & Company \\
\hline Coomassie blue G-250 & Thermo scientific, Rockford, USA \\
\hline DAPI mounting medium & Vector laboratories, Burlingame, CA \\
\hline DMEM & $\begin{array}{l}\text { Gibco }{ }^{\circledR} \text { by Life technologies ltd, } \\
\text { Paisley, UK }\end{array}$ \\
\hline Fast SYBR Green Master mix & Thermo scientific, Rockford, USA \\
\hline FBS & $\begin{array}{l}\text { Gibco }^{\circledR} \text { by Life technologies ltd, } \\
\text { Paisley, UK }\end{array}$ \\
\hline Gemcitabine hydrochloride & Sigma Aldrich, St. Louis, USA \\
\hline
\end{tabular}




\begin{tabular}{|l|l|}
\hline HBSS 1x & $\begin{array}{l}\text { Gibco }{ }^{\circledR} \text { by Life technologies ltd, } \\
\text { Paisley, UK }\end{array}$ \\
\hline LiberaseTL Research grade & Roche, Basel, Switzerland \\
\hline MEM AA solution (50x) & $\begin{array}{l}\text { Gibco }{ }^{\circledR} \text { by Life technologies ltd, } \\
\text { Paisley, UK }\end{array}$ \\
\hline MEM NEAA solution (50x) & $\begin{array}{l}\text { Gibco }{ }^{\circledR} \text { by Life technologies ltd, } \\
\text { Paisley, UK }\end{array}$ \\
\hline Midori green & $\begin{array}{l}\text { Nippon genetics Europe GmbH, } \\
\text { Germany }\end{array}$ \\
\hline MTT reagent & Sigma Aldrich, St. Louis, USA \\
\hline NuPAGE 4-12\% Bis-Tris gel & $\begin{array}{l}\text { Invitrogen by Thermo scientific, } \\
\text { Carlsbad, CA }\end{array}$ \\
\hline Penicillin/streptomycin & $\begin{array}{l}\text { Gibco }{ }^{\circledR} \text { Thermo scientific, Waltham, } \\
\text { USA }\end{array}$ \\
\hline RNA later ${ }^{\circledR}$ RNA stabilization reagent & Qiagen GmbH, Hilden, Germany \\
\hline SBTI & Sigma Aldrich, St. Louis, USA \\
\hline Trypsin EDTA (0.5\%) & $\begin{array}{l}\text { Gibco }{ }^{\circledR} \text { Thermo scientific, Waltham, } \\
\text { USA }\end{array}$ \\
\hline Protease inhibitor cocktail (25x complete) & Roche, Basel, Switzerland \\
\hline Dulbecco's PBS 1x & $\begin{array}{l}\text { Gibco }{ }^{\circledR} \text { Thermo scientific, Waltham, } \\
\text { USA }\end{array}$ \\
\hline
\end{tabular}

\subsection{Buffers and solutions}

\subsubsection{Buffers for primary cell isolation}

\section{Wash Buffer pH 6}

\begin{tabular}{|l|c|}
\hline \multicolumn{1}{|c|}{ Component } & For 1000ml \\
\hline HBSS & $770 \mathrm{ml}$ \\
\hline HEPES (1M) & $3.3 \mathrm{ml}$ \\
\hline MEM Amino Acids $(50 \mathrm{x})$ & $20 \mathrm{ml}$ \\
\hline MEM NEAA $(100 \mathrm{x})$ & $10 \mathrm{ml}$ \\
\hline Sodiumpyruvate $(100 \mathrm{mM})$ & $10 \mathrm{ml}$ \\
\hline Trisodiumcitrate $(0,68 \mathrm{M}), \mathrm{pH} 7.6$ & $10 \mathrm{ml}$ \\
\hline Glycerol & $2.1 \mathrm{ml}$ \\
\hline Magnesium chloride $(200 \mathrm{mM})$ & $135 \mu \mathrm{l}$ \\
\hline Calcium chloride $(100 \mathrm{mM})$ & $80 \mu 1$ \\
\hline H2O & Make up to $1 \mathrm{~L}$ \\
\hline
\end{tabular}

\section{Digestion buffer}

\begin{tabular}{|l|c|}
\hline \multicolumn{1}{|c|}{ Component } & For 100ml \\
\hline Wash Buffer $\mathrm{pH} 6$ & $97.5 \mathrm{ml}$ \\
\hline Liberase1 $(5 \mathrm{mg} / \mathrm{ml})$ & $2 \mathrm{ml}$ \\
\hline SBTI $(50 \mathrm{mg} / \mathrm{ml})$ & $0.5 \mathrm{ml}$ \\
\hline
\end{tabular}




\subsubsection{Buffers for genotyping}

Alkaline lysis buffer pH 12

\begin{tabular}{|c|c|}
\hline Component & Concentration \\
\hline $\mathrm{NaOH}$ & $25 \mathrm{mM}$ \\
\hline EDTA & $0.2 \mathrm{mM}$ \\
\hline
\end{tabular}

Neutralization buffer pH 5

\begin{tabular}{|l|c|}
\hline \multicolumn{1}{|c|}{ Component } & Concentration \\
\hline Tris. $\mathrm{HCl}$ & $40 \mathrm{mM}$ \\
\hline
\end{tabular}

TBE buffer (10X) pH 8.0

\begin{tabular}{|l|c|}
\hline \multicolumn{1}{|c|}{ Component } & For 1000ml \\
\hline Tris & $108 \mathrm{~g}$ \\
\hline Boric acid & $55 \mathrm{~g}$ \\
\hline EDTA & $7,44 \mathrm{G}$ \\
\hline $\mathrm{H}_{2} \mathrm{O}$ & Make up to $1 \mathrm{~L}$ \\
\hline
\end{tabular}

\subsubsection{Buffers for Western blot}

\section{Protein lysis buffer}

\begin{tabular}{|l|c|}
\hline \multicolumn{1}{|c|}{ Component } & Concentration \\
\hline HEPES pH 7.5-7.9 & $50 \mathrm{mM}$ \\
\hline $\mathrm{NaF}$ & $100 \mathrm{mM}$ \\
\hline $\mathrm{Na}_{4} \mathrm{O}_{7} \mathrm{P}_{2} \times 10 \mathrm{H}_{2} \mathrm{O}$ & $10 \mathrm{mM}$ \\
\hline $\mathrm{NaCl}$ & $150 \mathrm{mM}$ \\
\hline EGTA & $1 \mathrm{mM}$ \\
\hline Glycerin & $10 \%$ \\
\hline Triton X-100 & $1 \%$ \\
\hline
\end{tabular}

\section{Readymade Buffers}

\begin{tabular}{|l|l|c|}
\hline \multicolumn{1}{|c|}{ Stock } & Working solution & Company \\
\hline $\begin{array}{l}\text { NuPAGE } \\
\text { Sample buffer }(4 \mathrm{x})\end{array}$ & $\begin{array}{l}1: 4 \text { dilution }+10 \% \\
\beta-\mathrm{ME}\end{array}$ & $\begin{array}{c}\text { Invitrogen by Life } \\
\text { Technologies, Carlsbad, } \\
\text { CA }\end{array}$ \\
\hline $\begin{array}{l}\text { NuPAGE }{ }^{\circledR} \text { MOPS } \\
\text { SDS Running buffer } \\
(20 \mathrm{x})\end{array}$ & $\begin{array}{l}1: 20 \text { dilution in } \\
\text { distilled water }\end{array}$ & $\begin{array}{c}\text { Invitrogen by Life } \\
\text { Technologies, Carlsbad, } \\
\text { CA }\end{array}$ \\
\hline $\begin{array}{l}\text { NuPAGE } \\
\text { buffer }(20 \mathrm{x})\end{array}$ & $\begin{array}{l}1: 20 \text { dilution in } \\
\text { distilled } \\
\text { water+10\% } \\
\text { methanol }\end{array}$ & $\begin{array}{c}\text { Invitrogen by Life } \\
\text { Technologies, Carlsbad, }\end{array}$ \\
\hline
\end{tabular}


Wash Buffer

PBS $+0.1 \%$ Tween-20 (PBS-T)

\section{Blocking solution}

\begin{tabular}{|l|l|}
\hline \multicolumn{1}{|c|}{ Component } & \multicolumn{1}{c|}{ Concentration } \\
\hline PBS-T & $1 \mathrm{x}$ \\
\hline Non-fat milk & $5 \%$ \\
\hline
\end{tabular}

\subsubsection{Buffers for Immunohistochemistry}

Citrate buffer pH 6.0

\begin{tabular}{|l|l|}
\hline \multicolumn{1}{|c|}{ Component } & \multicolumn{1}{c|}{ Concentration } \\
\hline Citric acid monohydrate & $2.1 \mathrm{~g}$ \\
\hline $\mathrm{H}_{2} \mathrm{O}$ & Make up to 1L \\
\hline
\end{tabular}

TE buffer pH 9.0

\begin{tabular}{|l|l|}
\hline \multicolumn{1}{|c|}{ Component } & \multicolumn{1}{c|}{ Concentration } \\
\hline EDTA $(1 \mathrm{mM})$ & $0.372 \mathrm{~g}$ \\
\hline Tris & $1.211 \mathrm{~g}$ \\
\hline $\mathrm{H}_{2} \mathrm{O}$ & Make up to $1 \mathrm{~L}$ \\
\hline
\end{tabular}

TBS

\begin{tabular}{|l|l|}
\hline \multicolumn{1}{|c|}{ Component } & \multicolumn{1}{c|}{ Concentration } \\
\hline Tris- $\mathrm{HCl} \mathrm{pH} 7.4$ & $50 \mathrm{mM}$ \\
\hline $\mathrm{NaCl}$ & $150 \mathrm{mM}$ \\
\hline
\end{tabular}

TBS-T

TBS $+0.1 \%$ Tween- 20

Blocking solution

$10 \%$ Goat serum $+1 \%$ BSA in TBS-T

\subsection{Kits}

\begin{tabular}{|l|l|}
\hline \multicolumn{1}{|c|}{ Name } & \multicolumn{1}{c|}{ Company } \\
\hline $\begin{array}{l}\text { DNeasy } \\
\text { kit }\end{array}$ & Blood and Tissue \\
\hline $\begin{array}{l}\text { ImmPACT }{ }^{\mathrm{TM}} \text { DAB Peroxidase } \\
\text { Substrate kit (SK4105) }\end{array}$ & Vector laboratories, Burlingame, CA \\
\hline KAPA 2G fast HS & KAPA Biosystems, MA, USA \\
\hline Masson trichrome staining kit & Polysciences \\
\hline
\end{tabular}




\begin{tabular}{|l|l|}
\hline M.O.M ${ }^{\mathrm{TM}}$ Kit (BMK-2202) & Vector laboratories, Burlingame, CA \\
\hline Picrosirius red stain kit & Polysciences, Germany \\
\hline $\begin{array}{l}\text { Peroxidase Goat IgG kit (PK- } \\
\text { 4005)vectastain ABC kit }\end{array}$ & Vector laboratories, Burlingame, CA \\
\hline $\begin{array}{l}\text { Peroxidase Mouse IgG kit } \\
\text { (PK- 4002)vectastain ABC kit }\end{array}$ & Vector laboratories, Burlingame, CA \\
\hline $\begin{array}{l}\text { Peroxidase Rabbit IgG kit } \\
\text { (PK- 4001)vectastain ABC kit }\end{array}$ & Vector laboratories, Burlingame, CA \\
\hline $\begin{array}{l}\text { Peroxidase Rat IgG kit (PK- } \\
\text { 4004)vectastain ABC kit }\end{array}$ & Vector laboratories, Burlingame, CA \\
\hline Western Lighting ${ }^{\circledR}$ Plus-ECL & PerkinElmer, Inc., Waltham, USA \\
\hline
\end{tabular}

\subsection{Primers}

\section{Genotype primers}

\begin{tabular}{|l|l|}
\hline \multicolumn{1}{|c|}{ Gene name } & \multicolumn{1}{c|}{ Sequence (5'-3') } \\
\hline Cre (p48) forward & TGCTGTTTCACTGGTTATGCGG \\
\hline Cre (p48) reverse & TTGCCCCTGTTTCACTATCCAG \\
\hline K-ras mut forward & AGCTAGCCACCATGGCTTGAGTAAGTCTGCG \\
\hline K-ras mut reverse & CCTTTACAAGCGCACGCAGACTGTAGA \\
\hline SPARC Common forward & TTCTTCCTTGCAACCCTCTC \\
\hline SPARC wild type reverse & TGTGGAGCTTCCTCTGTCCT \\
\hline SPARC Mutant reverse & GGGGTTTGCTCGACATTG \\
\hline 1lox $<$ K-ras $>$ G12D forward & GGG TAG GTG TTG GGA TAG CTG \\
\hline 1lox $<$ K-ras $>$ G12D reverse & TCCGAATTCAGTGACTACAGATGTACAGAG \\
\hline
\end{tabular}

\subsection{Antibodies}

\subsubsection{Primary antibodies}

\begin{tabular}{|l|c|c|c|c|c|}
\hline Antibody & Company & Ref. Number & Clone & \multicolumn{2}{|c|}{ Dilution } \\
\cline { 5 - 6 } & & & & IHC & WB \\
\hline a-SMA & Dako & M0851 & Clone 1A4 & $1: 100$ & \\
\hline $\begin{array}{l}\text { Cytidine } \\
\text { deaminase }\end{array}$ & Abcam & Ab82346 & & $1: 100$ & \\
\hline CD31 & BD Pharmingen & 553370 & & $1: 100$ & \\
\hline CD45 & BD Biosciences & 550539 & & $1: 20$ & \\
\hline $\begin{array}{l}\text { Cleaved } \\
\text { Caspase-3 }\end{array}$ & Cell signaling & \#9664L & (D175)(5A1E) & $1: 100$ & \\
\hline Collagen I & Abcam & ab21286 & & $1: 100$ & \\
\hline CollagenIV & Abcam & ab19808 & & $1: 800$ & $1: 1000$ \\
\hline E-Cadherin & BD Biosciences & BD610181 & & & $1: 1000$ \\
\hline HSP-90 & Cell signaling & 48755 & E289 & & $1: 1000$ \\
\hline $\begin{array}{l}\text { Hyaluronic } \\
\text { acid }\end{array}$ & Calbiochem & 385911 & & $1: 100$ & \\
\hline
\end{tabular}




\begin{tabular}{|l|c|c|c|c|c|}
\hline $\begin{array}{l}\text { binding } \\
\text { protein }\end{array}$ & Thermo & RM-9106-50 & SP 6 & $1: 200$ & \\
\hline Ki-67 & $\begin{array}{c}\text { Scientific } \\
\text { company.Inc }\end{array}$ & C15296 & & $1: 100$ & \\
\hline NT5c1A & $\begin{array}{c}\text { Abcam } \\
\text { Pan- } \\
\text { cytokeratin }\end{array}$ & ab6401 & PCK-26 & $1: 700$ & \\
\hline SPARC & R\&D Systems & AF942 & & $1: 200$ & $1: 2000$ \\
\hline
\end{tabular}

\subsubsection{Secondary antibodies for western blot}

\begin{tabular}{|l|l|l|l|l|}
\hline \multicolumn{1}{|c|}{ Name } & \multicolumn{1}{|c|}{ Company } & \multicolumn{1}{c|}{$\begin{array}{c}\text { Ref. } \\
\text { Number }\end{array}$} & \multicolumn{1}{c|}{ WB } & IHC \\
\hline Rabbit anti-Goat (IgG)-HRP & Dako & P0449 & $1: 2000$ & \\
\hline Rabbit anti-Mouse (IgG)-HRP & Dako & P0161 & $1: 2000$ & \\
\hline Rabbit anti-Rat (IgG)-HRP & Dako & P0450 & $1: 2000$ & \\
\hline Swine anti-Rabbit (IgG)-HRP & Dako & P0217 & $1: 2000$ & \\
\hline Biotinylated anti-Goat IgG & Vectastain ABC kit & PK-4005 & & $1: 200$ \\
\hline Biotinylated anti-Mouse IgG & Vectastain ABC kit & PK-4002 & & $1: 200$ \\
\hline Biotinylated anti-Rat IgG & Vectastain ABC kit & PK-4001 & & $1: 200$ \\
\hline Biotinylated anti-Rabbit IgG & Vectastain ABC kit & PK-4004 & & $1: 200$ \\
\hline
\end{tabular}

2.8 Molecular weight standards

\begin{tabular}{|l|l|}
\hline \multicolumn{1}{|c|}{ Name } & \multicolumn{1}{c|}{ Company } \\
\hline Quick load ${ }^{\circledR} 100$ bp DNA ladder & New England Biolabs, Ipswich, USA \\
\hline $\begin{array}{l}\text { Precision plus protein Dual colour } \\
\text { standard }\end{array}$ & New England Biolabs, Ipswich, USA \\
\hline
\end{tabular}

\subsection{Cell lines}

Isolated primary PanIN and fibroblasts from $\mathrm{KC}^{-S P A R C} \mathrm{Ct}^{\mathrm{wt}}$ and $\mathrm{KC}^{-S P A R C} \mathrm{C}^{-/-}$were used in in vitro experiments of this study.

\subsection{Cell culture media}

$\mathrm{DMEM}+10 \% \mathrm{FBS}+1 \% \mathrm{NEAA}+1 \% \mathrm{P} / \mathrm{S}$

DMEM+10\% FBS+1\% NEAA

DMEM $+0.1 \%$ FBS $+1 \%$ NEAA 


\section{Methods}

\subsection{Mouse models}

$\mathrm{SPARC}^{\mathrm{wt}}, \mathrm{SPARC}^{-/-}, \mathrm{KC}^{-S P A R C}{ }^{\mathrm{wt}}$ and $\mathrm{KC}^{-S P A R C}{ }^{-/-}$mice with the genetic background of 129SvB6 were used in this study. SPARC ${ }^{\mathrm{wt}}$ and SPARC $\mathrm{SP}^{-/-}$mice were initially purchased from Jackson Laboratory. Generation of KC-SPARC ${ }^{\mathrm{wt}}$ and $\mathrm{KC}^{-S P A R C} \mathrm{C}^{-/}$mice was achieved through multiple breeding steps as previously shown by Hingorani et al., 2003.

Initially SPARC ${ }^{\mathrm{wt}}$ and SPARC $\mathrm{SP}^{-/}$mice were crossed with mice harbouring conditional LSL-Kras ${ }^{\mathrm{G} 12 \mathrm{D}}$ allele in which the expression of oncogenic $\mathrm{Kras}^{\mathrm{G} 12 \mathrm{D}}$ is inhibited by LoxSTOP-Lox cassette. Further, the expression of mutated oncogenic Kras was achieved by crossing $\mathrm{Kras}^{\mathrm{G} 12 \mathrm{D}(+/ \mathrm{T})}$; $\mathrm{SPARC}^{\mathrm{wt}}$ and $\mathrm{Kras}^{\mathrm{G} 12 \mathrm{D}(+/ \mathrm{T})}$; $\mathrm{SPARC}^{-/-}$mice with mice expressing Cre recombinase under the pancreas specific promoter $\mathrm{p} 48$.

Genotyping of the different mouse strains was performed by various genotyping protocols as discussed in part 3.3.3.

\subsection{Cell culture}

\subsubsection{Primary cell isolation}

Primary pancreatic cells were isolated from pre neoplastic lesions and tumour bearing $\mathrm{KC}-S P A R C^{\mathrm{wt}}$ and $\mathrm{KC}-\mathrm{SPARC}^{-/-}$mice using an established protocol. The protocol involves the dissociation of cells from collagen using Liberase. Liberase is an enzyme mixture in which Collagenase I and Collagenase II are mixed in an appropriate ratio together with Thermolysin at a low concentration. Upon mouse dissection $2-3 \mathrm{~mm}$ of pancreas tissue was taken and placed on a petri dish with PBS on ice. The following steps were performed under sterile condition in a cell culture safety cabinet. The tissue was then chopped into small pieces using a scalpel as quick as possible and $10 \mathrm{ml}$ of digestion buffer (buffer composition is mentioned in detail in the materials) containing Liberase and trypsin inhibitor was added and mixed well. The mixture was then incubated at $37^{\circ} \mathrm{C}$ for $10 \mathrm{~min}$. After incubation, the cells were collected in a $50 \mathrm{ml}$ falcon and centrifuged at $1200 \mathrm{rpm}$ for $5 \mathrm{~min}$ at room temperature (RT). After removing the supernatant carefully, the pellet was resuspended in 5-10 $\mathrm{ml}$ of digestion buffer. The mixture was centrifuged at $1200 \mathrm{rpm}$ for $5 \mathrm{~min}$ followed by incubation in the water bath at $37^{\circ} \mathrm{C}$ for $10 \mathrm{~min}$. The supernatant was removed carefully and the pellet was resuspended in washing buffer with $50 \mathrm{mg} / \mathrm{ml}$ of Soy Bean Trypsin Inhibitor (SBTI) to prevent cell lysis 
by trypsin. The cell suspension was then filtered through a $100 \mu \mathrm{m}$ cell strainer and centrifuged at 1200rpm for $5 \mathrm{~min}$. The cell pellet was resuspended in $10 \mathrm{ml}$ of DMEM supplemented with $10 \%$ FBS, $1 \%$ NEAA and $1 \% \mathrm{P} / \mathrm{S}$ and cultured in a $10 \mathrm{~cm}$ petri-dish. Medium change was given the next day and every second day afterwards. The resulting cell population was a mixture of adherent epithelial cells and fibroblasts. The mixed cell population was then subjected to serial trypsinization until it became as homogenous population of epithelial and fibroblasts cells.

\subsubsection{Expansion and culturing of primary cells}

When the isolated primary cells reached $70-80 \%$ of growth confluence, the medium was removed and the cells were washed with PBS once. Then $2 \mathrm{ml}$ of $0.5 \%$ trypsin EDTA per T75 culture flask was added and incubated at $37^{\circ} \mathrm{C}$ for $30 \mathrm{sec}-60 \mathrm{sec}$. As the fibroblasts are sensitive for trypsin treatment, they detach from plastic in a very short time of about $30 \mathrm{sec}$. The detached fibroblasts were cultured in a new culture flask with DMEM supplemented with $10 \%$ FBS, $1 \%$ NEAA.

The original flask was washed once with PBS to get rid any left detached fibroblasts and then fresh DMEM supplemented with 10\% FBS, and 1\% NEAA was added. This process of serial trypsinization was done until the epithelial and fibroblast cell populations reached homogeneity. The homogeneity of the culture was confirmed by microscopic observation, genotyping and western blot.

\subsubsection{Wound healing assay}

Wound healing assays are used to assess the migration of the cells in 2D based culture. The cells were grown in a 6 well plate until they reached $70-80 \%$ confluence. Subsequently, the cells were subjected to serum starvation for $24 \mathrm{~h}$ (serum concentration $0.1 \%$ ). The next day, the wound was introduced by making a precise scratch using a sterile $100 \mu 1$ micro tip across the middle of the wells. The migration was assessed by live cell imaging microscope (Leica DMi8) equipped with $\mathrm{CO}_{2}$ and $37^{\circ} \mathrm{C}$ temperature maintenance. The pictures were taken every $6 \mathrm{~h}$ till the wounds were completely closed. 


\subsection{Molecular biology techniques}

\subsubsection{DNA isolation from cultured cells}

DNA isolation from cultured cells was performed using DNeasy ${ }^{\circledR}$ Blood \& Tissue Kit from Qiagen. The protocol given by the manufacturer was followed. Maximum of $5 \times 10^{6}$ cells were collected from the culture flask and centrifuged at 190rpm for $5 \mathrm{~min}$. The supernatant was removed and cells were resuspended in $200 \mu 1$ of PBS, and $20 \mu 1$ proteinase $\mathrm{K}$ was added to this mixture. Then, $200 \mu 1$ of buffer AL (lysis buffer) was added to this and mixed thoroughly by vortexing. The mix was then incubated at $56^{\circ} \mathrm{C}$ for $10 \mathrm{~min}$. After the incubation $200 \mu \mathrm{l}$ of $96-100 \%$ of ethanol was added to this and mixed well by vortexing. The whole mixture was then transferred to a DNeasy Mini spin column placed on a $2 \mathrm{ml}$ collection tube provided in the kit. The column was then briefly centrifuged at $8000 \mathrm{rpm}$ for $1 \mathrm{~min}$. The collection tube with the flow through was discarded and the column was placed on a new $2 \mathrm{ml}$ collection tube. The column was then briefly washed with $500 \mu 1$ of AW1 buffer (wash buffer with low concentration of quanidine) and centrifuged at $8000 \mathrm{rpm}$. The flow through and the collection tube was discarded. The column was placed in a new $2 \mathrm{ml}$ collection tube and washed with $500 \mu \mathrm{l}$ of AW2 buffer (Tris- based wash buffer) and centrifuged at 14000rpm for $3 \mathrm{~min}$. The flow through and the collection tube was discarded. The column was then placed in a new $1.5 \mathrm{ml}$ mini centrifuge tube and $200 \mu \mathrm{AE}$ buffer (elution buffer) was added and incubated for $1 \mathrm{~min}$ at RT. The DNA was eluted by centrifuging the column at $8000 \mathrm{rpm}$ and stored at $-20^{\circ} \mathrm{C}$.

\subsubsection{DNA isolation from mouse tails for genotyping}

DNA extraction was done from tail tips of 3-4 weeks old mice for genotyping. 2-3 mm of mouse tail tips were collected and the tissue lysis was achieved by heating the tail biopsy in $100 \mu \mathrm{l}$ of alkaline lysis buffer for $30 \mathrm{~min}$ at $95^{\circ} \mathrm{C}$. The lysates were then brought to RT and $100 \mu 1$ of neutralization buffer was added. At this step, the tail tips look visibly intact but the DNA will be released into the supernatant.

\subsubsection{Genotyping}

The protocols for SPARC, Kras ${ }^{\mathrm{G} 12 \mathrm{D}}$ and Cre-genotyping were obtained from the Jackson laboratory. 


\subsubsection{SPARC genotyping}

The reaction mix was prepared as given in the original protocol from the Jackson Laboratory by mixing $12.5 \mu \mathrm{l}$ of KAPA $2 \mathrm{G}$ Fast HS with $8.5 \mu 1 \mathrm{H}_{2} \mathrm{O}$ and $20 \mathrm{pM}$ of a common forward, a wild type reverse and a mutant reverse primer each. The triple primer system was used in order to detect WT, heterozygous and homozygous genotypes. $2 \mu 1$ of DNA isolated from tail tip was added to the reaction mix and the following steps were performed in the thermal cycler.

\begin{tabular}{|c|c|c|c|}
\hline Step & Temperature profile & Time & Note \\
\hline 1 & $94^{\circ} \mathrm{C}$ & $3 \mathrm{~min}$ & \\
\hline 2 & $94^{\circ} \mathrm{C}$ & $20 \mathrm{sec}$ & \multirow{2}{*}{35 cycles } \\
\hline 3 & $65^{\circ} \mathrm{C}$ & $15 \mathrm{sec}$ & \\
\hline 4 & $72^{\circ} \mathrm{C}$ & $15 \mathrm{sec}$ & \\
\hline 5 & $72^{\circ} \mathrm{C}$ & $2 \mathrm{~min}$ & \\
\hline 6 & $10^{\circ} \mathrm{C}$ & Infinite hold & \\
\hline
\end{tabular}

\subsubsection{Kras $^{\mathrm{G} 12 \mathrm{D}}$ and Cre genotyping}

Similar protocols for the reaction mix preparation and the thermal profile were used in $\operatorname{Kras}^{\mathrm{G} 12 \mathrm{D}}$ and Cre genotyping. For Kras ${ }^{\mathrm{G} 12 \mathrm{D}}$ genotyping the primers specific for mutated $\mathrm{Kras}^{\mathrm{G} 12 \mathrm{D}}$ were used. The reaction mix was prepared as given in the original protocol by adding $12.5 \mu \mathrm{l}$ of KAPA $2 \mathrm{G}$ Fast HS with $8.5 \mu 1 \mathrm{H}_{2} \mathrm{O}$ and $10 \mu \mathrm{M}$ of forward and reverse primers each. The reaction mix was then subjected to the following thermal profile using a thermal cycler.

\begin{tabular}{|l|l|l|l|}
\hline Step & Temperature profile & \multicolumn{1}{|c|}{ Time } & \multirow{2}{*}{ Note } \\
\hline 1 & $95^{\circ} \mathrm{C}$ & $2 \mathrm{~min}$ & \\
\hline 2 & $95^{\circ} \mathrm{C}$ & $30 \mathrm{sec}$ & \multirow{2}{*}{34 cycles } \\
\hline 3 & $60^{\circ} \mathrm{C}$ & $30 \mathrm{sec}$ & \\
\hline 4 & $72^{\circ} \mathrm{C}$ & $1 \mathrm{~min}$ & \\
\hline 5 & $72^{\circ} \mathrm{C}$ & $5 \mathrm{~min}$ & \\
\hline 6 & $4^{\circ} \mathrm{C}$ & Infinite hold & \\
\hline
\end{tabular}

\subsubsection{1lox $-<$ K-ras $>$ G12D genotyping}

1 lox $<\mathrm{K}$-ras $>\mathrm{G}^{12 \mathrm{D}}$ genotyping was used to validate the homogeneity of isolated primary epithelial and fibroblast cells. The reaction mix was prepared by mixing $2 \mu 15 \mathrm{X}$ advantage GC melt mix, $0.4 \mu$ l of 50X advantage GC-2 polymerase mix, $4 \mu 1$ of $5 \mathrm{X}$ GC2 PCR buffer, 24pM of each forward and reverse primers, $4 \mu 1$ of $25 \mathrm{mM}$ d' NTP's and 11.2 $\mu 1$ of $\mathrm{H}_{2} \mathrm{O}$. The PCR reaction was performed in the thermal cycler as shown below. 


\begin{tabular}{|l|l|l|c|}
\hline Step & \multicolumn{1}{|c|}{ Temperature profile } & \multicolumn{1}{|c|}{ Time } & Note \\
\hline 1 & $94^{\circ} \mathrm{C}$ & $3 \mathrm{~min}$ & \\
\hline 2 & $94^{\circ} \mathrm{C}$ & $30 \mathrm{sec}$ & \multirow{2}{*}{40 cycles } \\
\hline 3 & $68^{\circ} \mathrm{C}$ & $1.5 \mathrm{~min}$ & \\
\hline 4 & $68^{\circ} \mathrm{C}$ & $3 \mathrm{~min}$ & \\
\hline
\end{tabular}

\subsubsection{Agarose gel electrophoresis}

Agarose gel electrophoresis is a widely used technique in molecular biology laboratories. This technique was originally invented by Oliver Smithies in 1950 and is used to separate the nucleic acids by their length.

Followed by PCR reaction, the amplified PCR product was run in 1.25-1.5\% of agarose gel using gel electrophoresis technique with Tris-Borase-EDTA (TBE) buffer at 100V for $20 \mathrm{~min}$. 100bp DNA ladder was used as a control.

\subsection{Protein techniques}

\subsubsection{Protein extraction from cultured cells}

Cells were cultured on a 6 well plate and washed with cold 1x PBS twice. Depending on cell density, 30-200 $\mu 1$ of protein lysis buffer containing protease and phosphatase inhibitors was added per well. The cells were scrapped and collected in a micro centrifuge tube and subjected to a short sonication to improve the protein yield. Followed by incubation on ice for 20-30 min, the lysate was then centrifuged at $14000 \mathrm{rpm}$ at $4{ }^{\circ} \mathrm{C}$ for $15 \mathrm{~min}$. The supernatant was collected in a new micro centrifuge tube and stored at $-20^{\circ} \mathrm{C}$ for a short period, and for longer storage at $-80^{\circ} \mathrm{C}$.

\subsubsection{Sample preparation}

The protein concentration was determined by Bradford method using Coomassie Blue G-250 protein assay reagent and known concentration of albumin as standard curve. Then $1 \mu \mathrm{g} / \mu 1$ of protein lysate was mixed with $4 \mathrm{xNuPAGE}^{\circledR}$ LDS sample buffer with $10 \% \beta$ Mercaptoethanol and boiled at $95^{\circ} \mathrm{C}$ for $5 \mathrm{~min}$.

\subsubsection{SDS-PAGE}

The samples were then subjected to poly acrylamide gel electrophoresis (PAGE) using NuPAGE 4-12\% Bis-Tris gel and Invitrogen mini gel tank. Minimum concentration of $20 \mu \mathrm{g}$ of protein was loaded per well.The separation of proteins was achieved by running the samples at $160 \mathrm{~V}$ for $1 \mathrm{~h}$ in $1 \mathrm{x}$ running buffer containing Sodium dodecyl sulphate (SDS) as a denaturing agent. 


\subsubsection{Western blot analysis}

Followed by the separation of protein by SDS-PAGE, the proteins were blotted on a nitrocellulose membrane using 1xNuPAGE transfer buffer with 10\% methanol. The resolved proteins from the gel were transferred to the nitrocellulose membrane using a transfer apparatus at $10 \mathrm{~V}$ for $1.5 \mathrm{~h}$. The membrane was blocked with $5 \%$ non-fatty milk for $1 \mathrm{~h}$ at RT to avoid unspecific binding of the primary antibody. Both primary and secondary antibodies were diluted in 5\% non-fat milk in PBS containing $0.1 \%$ Tween20. The membrane was incubated with the appropriate concentration of primary antibodies (concentrations of antibodies are mentioned in materials section) at $4{ }^{\circ} \mathrm{C}$ overnight. The following day, the membrane was washed 3 times with 1X PBS and then incubated with1:2000 dilution of secondary antibody conjugated with horseradish peroxidase for $1 \mathrm{~h}$ at RT. The membrane was washed 3 times with PBS-T to get rid of unbound secondary antibody. The proteins on the membrane were detected using Enhanced Chemi Luminescence (ECL). ECL is a technique which detects the reactivity of horseradish peroxidase with its substrate luminol as light emission and thus detects immobilized proteins on the membrane. The imaging was done using INTAS- ECL Chemocam imager and analysed using the software provided by the equipment provider.

\subsection{Histology}

\subsubsection{Tissue collection and processing}

Upon mouse biopsy, tissues such as pancreas, liver, spleen and intestines were collected in a glass vial containing $4 \%$ formaldehyde. The tissue samples were fixed with $4 \%$ formaldehyde overnight at RT. Fixation is the process which enhances the tissue stability by cross linking macromolecules and thus inhibiting the disintegration of biological materials. Following overnight fixation, the tissues were subjected to a dehydration process by treating them with a gradually increasing concentration of ethanol from 70 $99 \%$ at regular time intervals. The dehydration process was achieved using automated dehydration machine provided by Leica. After the dehydration process, the tissues were embedded in paraffin using tissue embedder system. The tissues in paraffin blocks were sectioned with the thickness of $4 \mu \mathrm{m}$ using a microtome (Leica RM2265) and fixed on SUPERFROST $^{\circledR}$ microscope glass slides and used for various histological staining protocols. 


\subsubsection{Haematoxylin and Eosin stain}

Haematoxylin and Eosin (H\&E) stain is one of the most commonly used dyes to study the basic histology of the tissues. The tissue sections were immersed in roticlear for 10 min two times in order to de-mask the paraffin. The tissue slides were subsequently subjected to rehydration process by treating them with gradually decreasing concentration of ethanol from $99-50 \%$ each for $3 \mathrm{~min}$. The tissue slides were then washed briefly in tap water 2-3 times and stained with haematoxylin for 5-7 min. Haematoxylin is a chemical compound which specifically stains cell nuclei in purple colour. The slides were washed with running tap water for 5 min until the colour turns purple. Then the slides were counterstained with eosin, a red stain specific for cytoplasm of the cell for 7 min. The slides were washed three times with tap water and the tissue was dehydrated by treating them with increasing concentration of ethanol from 70-99\% each for $3 \mathrm{~min}$. Finally, the slides were treated with roticlear 4 times each for $10 \mathrm{~min}$ and mounted using rotimount solution.

\subsubsection{Immunohistochemistry}

For immunohistochemical analysis, the tissue slides were deparaffinized and rehydrated as mentioned above in the H\&E staining protocol. Antigen retrieval was achieved by boiling the slides with appropriate buffer solutions (Tris-EDTA-pH 9.0 or Citrate buffer$\mathrm{pH}$ 6.0) for $10 \mathrm{~min}$ in the microwave at maximum power ( 700 watts). The slides were then cooled down on ice for $20 \mathrm{~min}$ and washed briefly with tap water. Then the slides were treated with freshly prepared $3 \% \mathrm{H}_{2} \mathrm{O}_{2}$ for 20 min to quench the endogenous peroxidase activity. Subsequently, the slides were rinsed in tap water for $5 \mathrm{~min}$ and aligned in a Cadenza slide holder system and washed thrice with TBS containing $0.1 \%$ Tween-20. Tween is a mild detergent used in many washing buffers. The tissues were then blocked with $10 \%$ of appropriate serum and 1\% BSA in TBS-T for 1 hour at RT. Both primary and secondary antibodies were diluted in the blocking solutions. The tissues were then incubated with appropriate concentration (antibody concentration was given in materials) of primary antibody at $4^{\circ} \mathrm{C}$ overnight. The following day, the slides were washed thrice with TBS-T before incubating them with biotinylated secondary antibody provided in a species specific $\mathrm{ABC}$ vectastain kit for 1 hour at RT. Afterwards, the slides were washed thrice with TBS-T and incubated with AB complex prepared by mixing equal volumes of Solution A and Solution B containing peroxidase (i.e. $10 \mu 1$ of Solution A and $10 \mu 1$ of Solution B) provided in the same kit. The slides were washed 
thrice with TBS-T before removing them from Cadenza system and once with tap water before treating them with 3,3'-Diaminobenzidine (DAB) a substrate of the peroxidase enzyme. The positive staining was observed as a brown chromogen development upon incubation with DAB.

\subsubsection{DAPI staining}

After de-waxing and rehydration of the tissue slides, the antigen retrieval was achieved by boiling the slides with appropriate buffer solutions (Tris-EDTA-pH 9.0 or Citrate buffer-pH 6.0) for $10 \mathrm{~min}$ in the microwave at maximum power (700 watts). The slides were cooled down on ice for $20 \mathrm{~min}$ and washed briefly with tap water 2-3 times. The slides were then aligned in a Cadenza system and washed thrice with TBS containing $0.1 \%$ Tween-20. Afterwards, the tissues were blocked with $10 \%$ of appropriate serum and $1 \%$ BSA in TBS-T for $1 \mathrm{~h}$ at RT before incubation with the appropriate concentration of primary antibody at $4{ }^{\circ} \mathrm{C}$ overnight. For immunofluorescence the appropriate secondary antibodies coupled with fluorochromes were used and the detection was achieved by using a fluorescence microscope.

\subsubsection{Masson trichrome staining}

Masson trichrome is a chemical staining used to detect the collagen fibers in tissue specimen embedded in paraffin. For masson trichrome staining, different compartments of tissues were stained in three different colours. The collagen fibers are recognized from other compartments by a prominent blue colour stain, while the nucleus is stained in black and the muscle and other parts stained in red. The Masson trichrome staining kit from Polysciences, Inc was used. The protocol was performed under the fume hood in order to avoid health hazards. The de-waxing and rehydration of slides was done as mentioned above. The fixation was achieved by incubating the slides overnight with Bouin's solution at RT. The slides were washed with running tap water for $5 \mathrm{~min}$ to remove picric acid. Subsequently, the slides were stained with Weigert's iron haematoxylin working solution (prepared by mixing 1:1 ratio of Weigert's haematoxylin A and Weigert's haematoxylin B) for $15 \mathrm{~min}$ and washed in running tap water for $5 \mathrm{~min}$ and rinsed once with distilled water. The slides were further incubated with Biebrich Scarlet - Acid Fuchsin Solution for $5 \mathrm{~min}$ and rinsed in distilled water. The slides were then treated with phosphomolybdic acid for 10 minutes, and directly transferred into a glass beaker containing aniline blue for $5 \mathrm{~min}$. Followed by rinsing with distilled water; the slides were treated with $1 \%$ acetic acid for $1 \mathrm{~min}$ and rinsed in distilled water. 
Subsequently, the slides were dehydrated with $95 \%$ and $99 \%$ of ethanol each for $30 \mathrm{sec}$ and cover slipped.

\subsubsection{Herovici staining}

Herovici is a chemical staining method used to distinguish young (pro) collagen from mature dense collagen (Friend, 1963). Herovici is a defined combination of methyl blue (aniline blue) and picro acid fuchsin. When the prestained sections are treated acetic acid or acidic water, the red stain is retained by the mature collagen while procollagen loses the red stain and turns into blue (Lillie et al., 1980). The cytoplasm and nucleus are counter stained yellow and black in colour respectively. The protocol provided by the Histopathology/ISH core facility, Cambridge research Institute was followed. The tissue slides were de-waxed and rehydrated as mentioned before and washed briefly in tap water before treating them with Weigert's hematoxylin solution for $5 \mathrm{~min}$. The slides were then briefly rinsed with tap water for $45 \mathrm{sec}$ and treated with Herovici's working solution (1:1 Herovici's solution A and Herovici's solution B) for 2 min. The slides were then directly kept in $1 \%$ acetic acid solution for 2 min without rinsing. Finally, the slides were dehydrated, and coverslipped.

\subsubsection{Picrosirius Red stain}

Picrosirius red stain is a chemical staining used to detect the total collagen in the given tissue. The Picrosirius red stain kit and the protocol from Polysciences, Inc was used. The slides were subjected to de-waxing and dehydration as mentioned before and treated with Weigert's haematoxylin solution for $5 \mathrm{~min}$. The slides were rinsed with tap water once and agitated with $2 \%$ acid ethanol for $10 \mathrm{sec}$. The slides were again rinsed with tap water and washed once with distilled water before counter staining with solution A (phosphomolybdic acid) provided in the kit for $2 \mathrm{~min}$. Subsequently, the slides were washed with distilled water and placed in solution B (picrosirius red F3BA stain) provided in the kit for 110 min without rinsing the slides. Afterwards, the slides were treated with solution $\mathrm{C}(0.1 \mathrm{~N} \mathrm{HCl})$ for $2 \mathrm{~min}$. Then the slides were treated with $70 \%$ ethanol for $45 \mathrm{sec}$ and further dehydrated and mounted.

\subsection{In vivo experiments}

The animal breeding and survival study (animal allowance application number -TVA14/1634) and other in vivo experiments (gemcitabine treatment- TVA15/2056) of our study have met the ethical requirements of the institute and the federal government. The 
animals were bred and maintained in Central animal experimental facility, University Medical Center Göttingen (ZTE-UMG). The animals were subjected to 12/12h day/night cycles. The newly born pups were separated from mother after three weeks of birth.

A clear score sheet with specific parameters (severity level 1-3) was followed to define the end point criteria. Severity level 1 includes isolation of animal in the cage, rough fur, narrowed ocular margins, slightly increased breathing, and alteration in the fecal /urine quantity, color and consistency. These animals were observed closely for recovery, and they are sacrificed if were found with persistent symptoms. Severity level 2 parameters include conditions such as persistant pain (facial expression, bent position), open wounds, severe breathing and diarrhea, notable weight loss (10\%). Animals with severity level 2 were sacrificed upon veterinarian's advice. While animals with severity level 3 symptoms (paralysis, any other terminal illness and significant weight loss (20\%) were immediately sacrificed. The animals that died of any symptoms mentioned above are considered as end point criteria. If the animals presented no symptoms at the age of 20 months, they were sacrificed and considered as end point.

\subsubsection{Study design and sample collection}

$\mathrm{KC}-S P A R C^{\mathrm{wt}}$ and $\mathrm{KC}^{-S P A R C} \mathrm{C}^{-/-}$mice were categorized into three different age groups: 3 months, 7-8 months and 12-20 months in order to study the role of SPARC during different stages of PDAC progression. Tissue samples such as pancreas, liver, spleen and intestine were harvested in $4 \%$ formaldehyde for histological analysis. For protein extraction, tissues such as pancreas, liver and intestine were snap frozen using liquid nitrogen. For RNA isolation pancreas and liver were collected in a vial containing RNA later, a stabilizing reagent.

\subsubsection{Gemcitabine treatment and the sample preparation for Mass Spectrometry analysis}

PanIN lesions bearing (7-8 months old) and tumor bearing $\mathrm{KC}^{-S P A R C}{ }^{\mathrm{wt}}$ and $\mathrm{KC}$ $\mathrm{SPARC}^{-/-}$mice and 3 months old SPARC ${ }^{\mathrm{wt}}$ and SPARC $\mathrm{SP}^{-/-}$(as control) cohorts were treated with $100 \mathrm{mg} / \mathrm{kg}$ of Gemcitabine for $2 \mathrm{~h}$ by an intraperitoneal injection. After $2 \mathrm{~h}$, the mice were sacrificed and the pancreas was harvested and instantly frozen by immersing the tissue in liquid nitrogen. The drug injections and sample collection were done by Ms. M. Patzak with the assistance of technical assistants. The pancreas tissue was processed and subjected to liquid chromatography-mass spectrometry/ mass spectrometry (LC-MS/MS) 
to analyse the concentration of native $2^{\prime}, 2^{\prime}$-difluorodeoxycytidine $(\mathrm{dFdC})$ and its metabolites such as $2^{\prime}, 2^{\prime}$-difluorodeoxycytidine-5'-triphosphate (dFdCTP) and $2^{\prime}, 2^{\prime}$ difluorodeoxyuridine (dFdU) as previously described (Bapiro et al., 2011). The LCMS/MS analysis was performed in CRUK-Pharmacokinetics \& Bioanalytics (PKB) Core Facility, Cancer Research UK Cambridge Institute. 


\section{Results}

In this study, we explored the expression of SPARC in normal murine pancreas and during various stages of disease initiation and progression to PDAC in the context of oncogenic Kras ${ }^{\mathrm{G} 12 \mathrm{D}}$. To experimentally address the above mentioned issues, I employed $\mathrm{KC}^{-S P A R C}{ }^{-/-}$and KC-SPARC ${ }^{\mathrm{wt}}$ GEMMs in this study. SPARC ${ }^{\mathrm{wt}}$ and $\mathrm{SPARC}^{-/-}$mice were used as controls.

\subsection{Characterization of mouse models}

The mouse models were successfully generated as mentioned previously in the methods section and observed closely for any noticeable phenotypes. Genetic ablation of SPARC has been reported to be lethal in the lower organisms such as C. elegans and Drosophila (Fitzgerald and Schwarzbauer, 1998; Martinek et al., 2011). Nevertheless, embryonic SPARC knockout is not lethal in mice according to the previous studies. In the absence of SPARC in higher animals, the functions of SPARC are partly accomplished by other members of the SPARC protein family (Liu et al., 2008; Sullivan and Sage, 2004).

$\mathrm{SPARC}^{-/-}$mice showed certain specific non-lethal phenotypes such as fragile bones, cataract and curled tail tips. These phenotypic changes are caused by impairment in collagen maturity and deposition. SPARC has been reported to aid the folding and deposition of collagen through various mechanisms, and thus the lack of SPARC resulted in low bone density (Bradshaw, 2009). SPARC has also been shown to maintain the lens fiber and fluid transport, thus the absence of SPARC affects the lens homeostasis (Greiling et al., 2009; Norose et al., 1998a).

3 months old $\mathrm{SPARC}^{-/-}(\mathrm{n}=6)$ and WT $(\mathrm{n}=6)$ mice were sacrificed and organs such as pancreas, liver, kidney and spleen were harvested and weighed. The whole body weight was measured prior to necropsy. The study results show that $\mathrm{SPARC}^{-/-}$mice have a trend towards reduced overall body weight compared to WT mice (Fig. 4A) $(p=0.06)$. Additionally, the weight of internal organs such as pancreas (Fig.4B) $(p=0.11)$, liver (Fig.4C) $(\mathrm{p}=0.13)$, kidney (Fig.4D) $(\mathrm{p}=0.02)$ and spleen (Fig.4E) $(\mathrm{p}=0.02)$ of $\mathrm{SPARC}^{-/-}$ mice were relatively lower than of WT mice. Though the difference was not statistically significant, the trend was obvious in terms of body weight, pancreas and liver weight between $\mathrm{SPARC}^{-/-}$and WT. 
A.

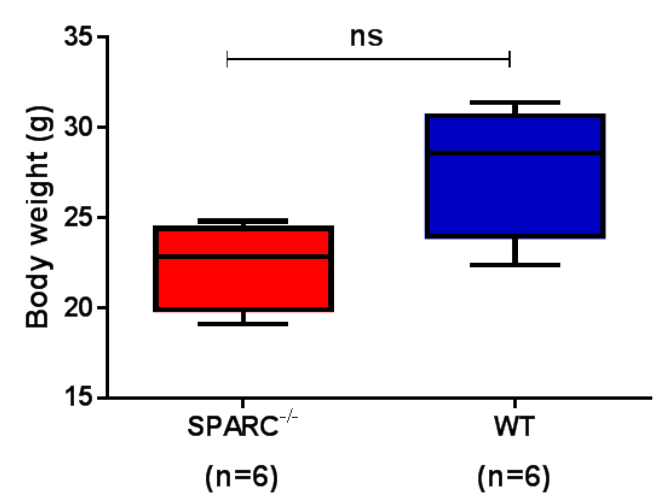

C.

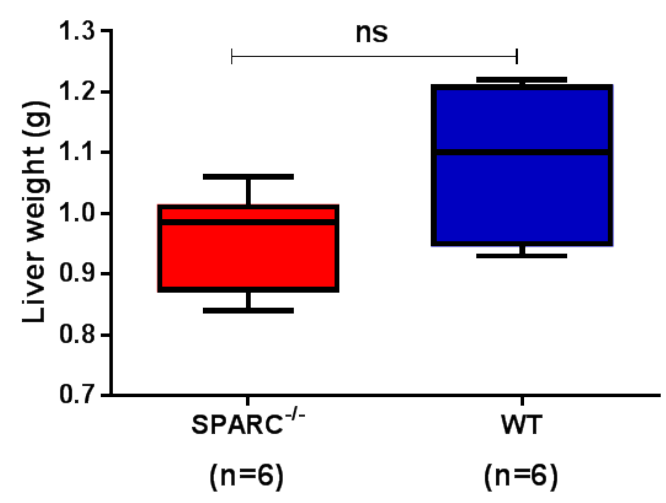

B.

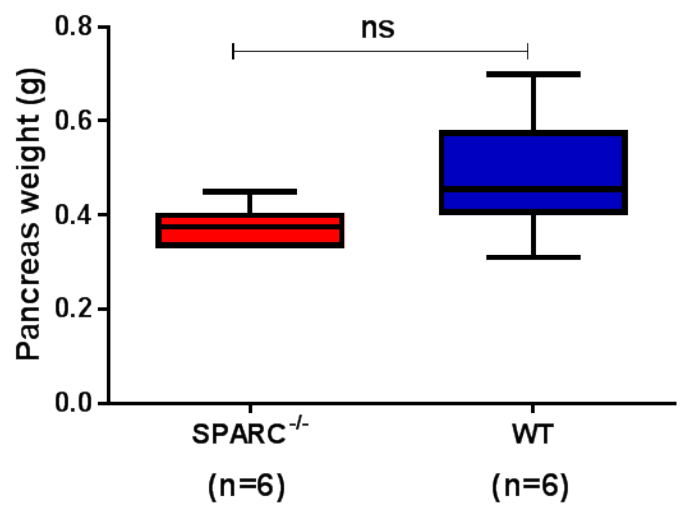

D.

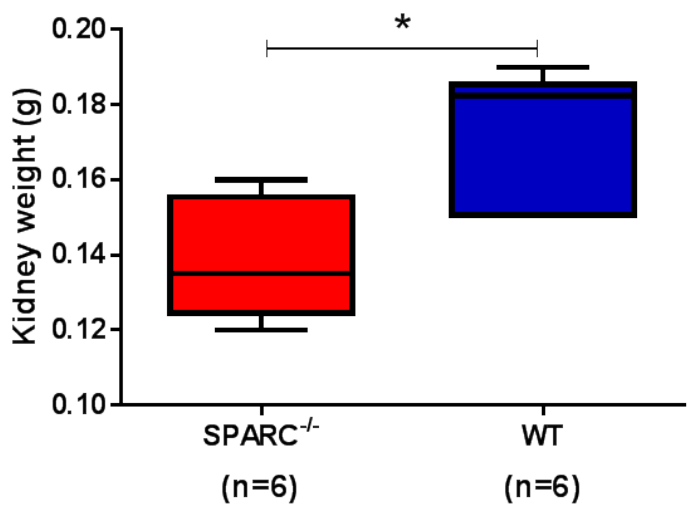

E.

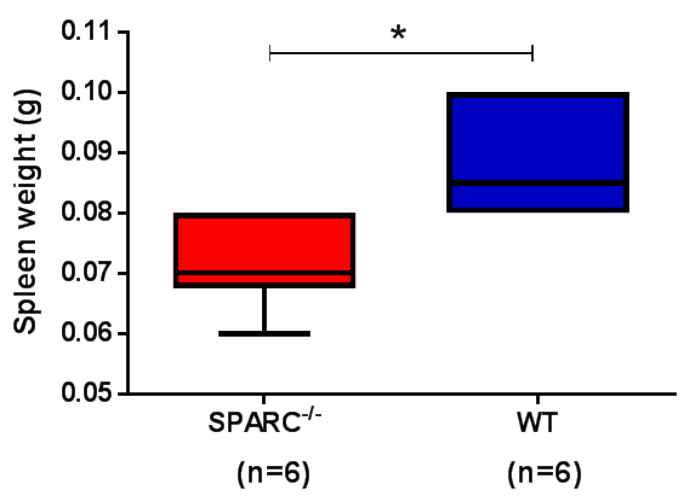

Figure 4: (A) Graph shows that SPARC ${ }^{-/-}$mice have a trend towards reduced overall body weight $(\mathrm{p}=0.06)$ compared with WT mice. (B, C, D \& E) Graphs showing that the weight of the internal organs such as pancreas $(\mathrm{p}=0.11)$, liver $(\mathrm{p}=0.13)$, kidney $(\mathrm{p}=0.02)$ and spleen $(\mathrm{p}=0.02)$ are also lower in SPARC $\mathrm{C}^{-/}$mice compared to WT mice. Mann-Whitney test was used to assess the statistical significance. ${ }^{*} \mathrm{p} \leq 0.05$. 


\subsubsection{SPARC knock out does not affect murine pancreas development}

Histology of pancreas assessed by H\&E staining confirmed that the murine pancreas development was not affected by genetic SPARC ablation. Pancreas tissue sections from 3 months old SPARC $^{-/-}$and WT mice stained with H\&E (Fig. 5) show the tissue integrity of the organ. Different compartments of the pancreas such as acinar cells (*) and islets (arrow) are found to be structurally intact.
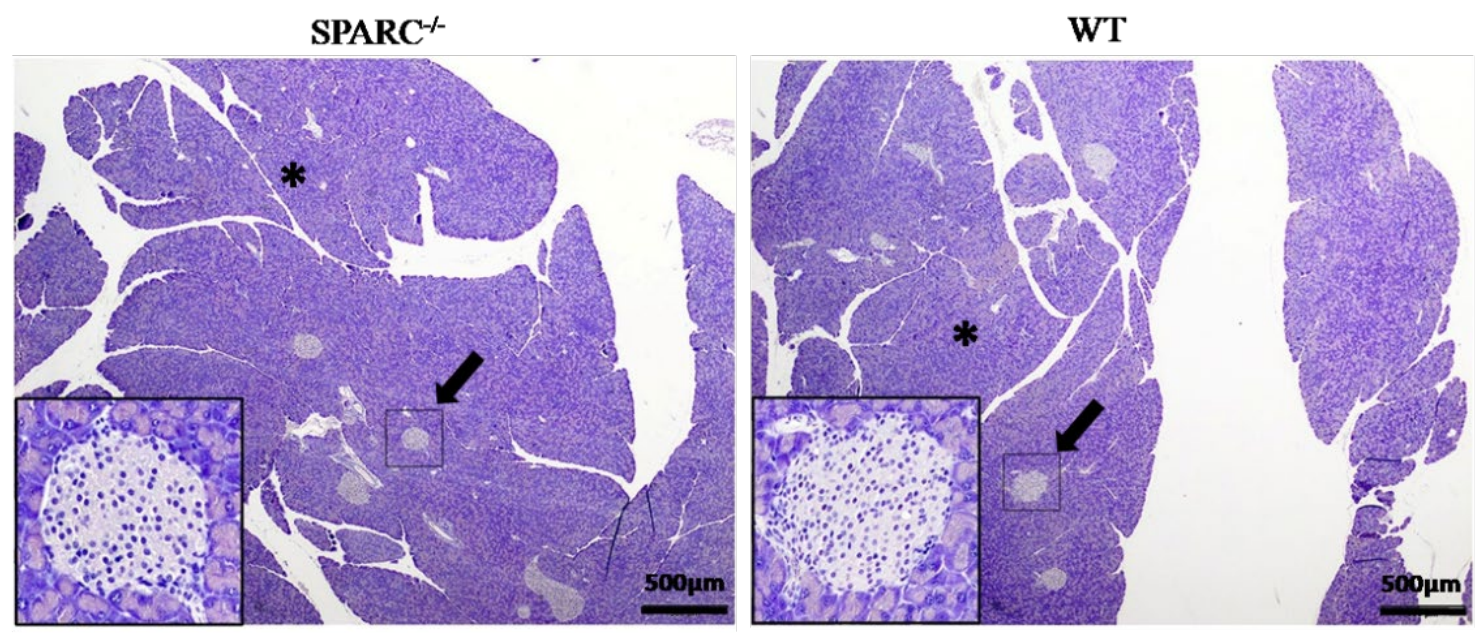

Figure 5: Representative images of H\&E staining of 3 months old SPARC ${ }^{-/-}$and WT mice pancreata. The inset shows higher magnification of islets.

\subsubsection{Oncogenic Kras ${ }^{\mathrm{G} 12 \mathrm{D}}$ activation results in disease initiation}

Upon oncogenic $\mathrm{Kras}^{\mathrm{G} 12 \mathrm{D}}$ expression both $\mathrm{KC}-\mathrm{SPARC}^{-/-}$and $\mathrm{KC}-\mathrm{SPARC}^{\mathrm{wt}}$ mice developed ADMs and low grade PanIN lesions at the age of 3 months (Fig. 6A) which further progressed to advanced PanIN lesions (Fig. 6B). Based on the anticipated progression stage, mice have been categorized under three age groups: 3-5 months (early prenneoplasia), 7-8 months (late preneoplasia and more than 12 months (tumors, end point cohort) in order to study the effect of SPARC on different stages of PDAC progression. 


\section{Results}

A.

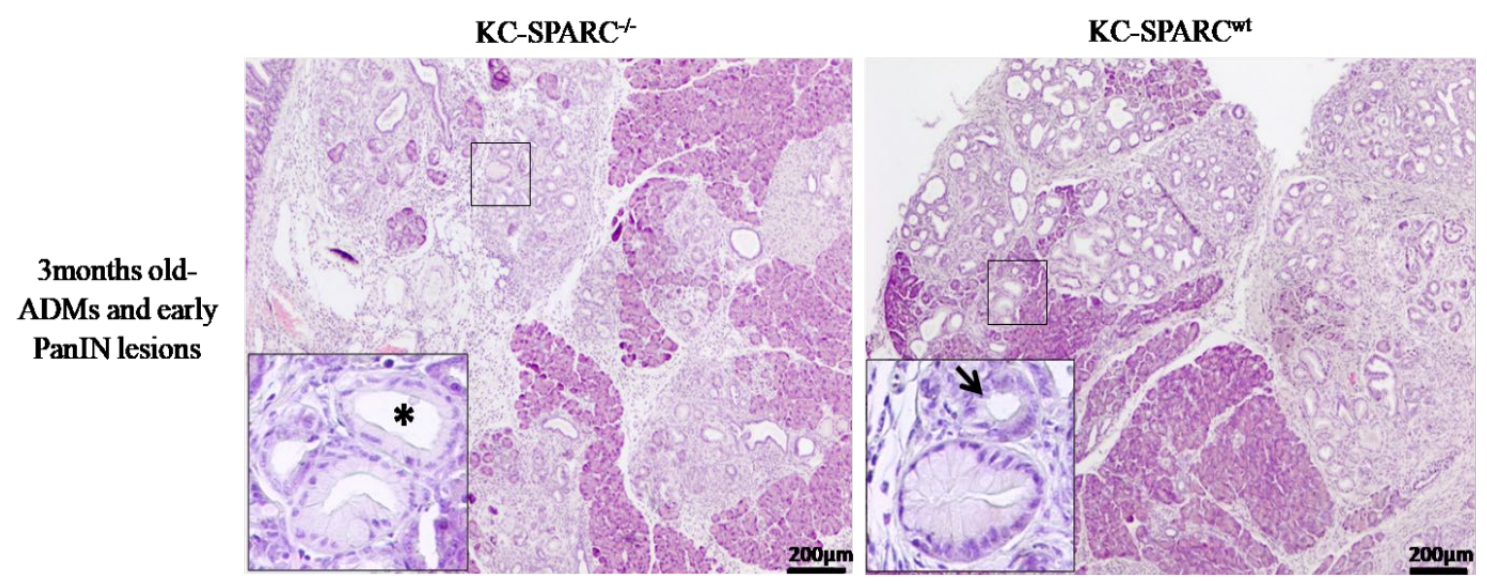

B.

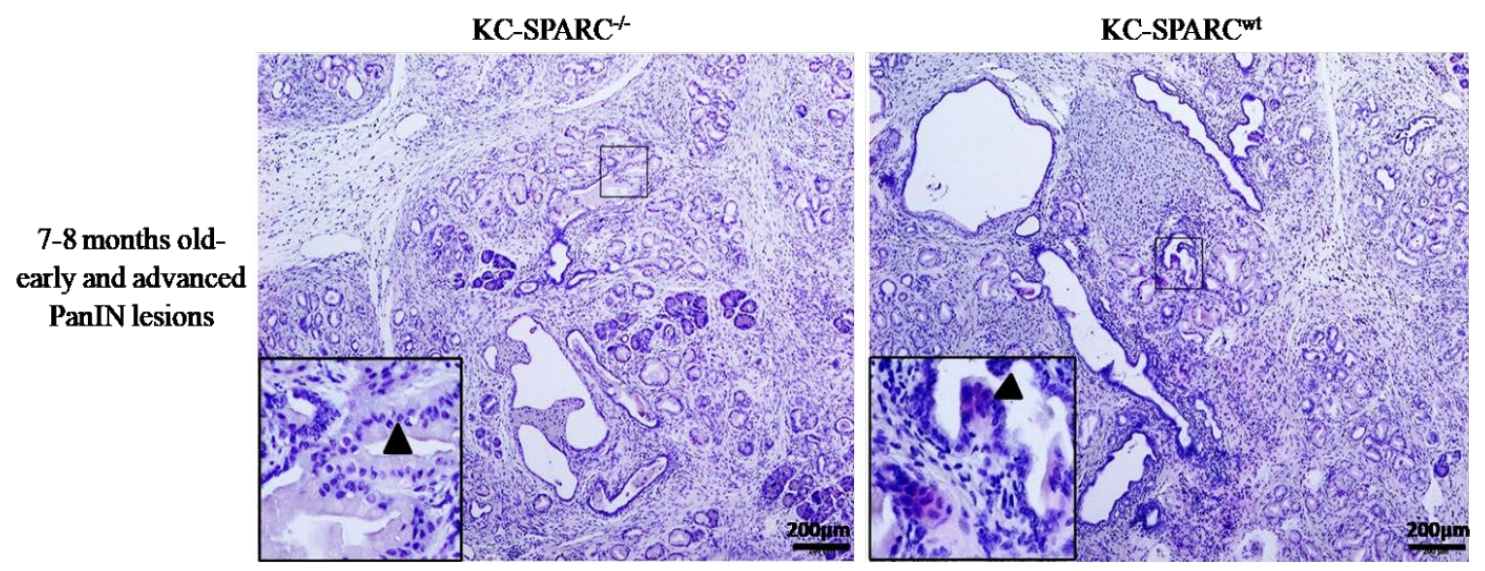

Figure 6: (A) Representative images of H\&E staining of 3 months old and (B) 7-8 months old KC-SPARCI- and KC-SPARC ${ }^{\mathrm{wt}}$ mice pancreata show ADMs (down arrow), low grade PanINs (*) and high grade PanINs (thick arrow head) respectively. The inset shows 40x magnification of ADMs and low PanINs and advanced PanIN lesion. 


\subsection{Primary cell isolation and characterization}

Primary PanIN associated fibroblasts (PAFs) and PanIN (epithelial) cells were successfully isolated from 3-5 months old $\mathrm{KC}-S P A R C^{\mathrm{wt}}$ and $\mathrm{KC}^{-S P A R C}{ }^{-}$and established in cell culture. Microscopic observation of the cells was performed to investigate the phenotypic characteristics of the isolated cells. Isolated fibroblasts exhibited spindle shaped elongated structure while the PanIN cells were growing in patches indicating the morphological fibroblast and epithelial properties respectively.

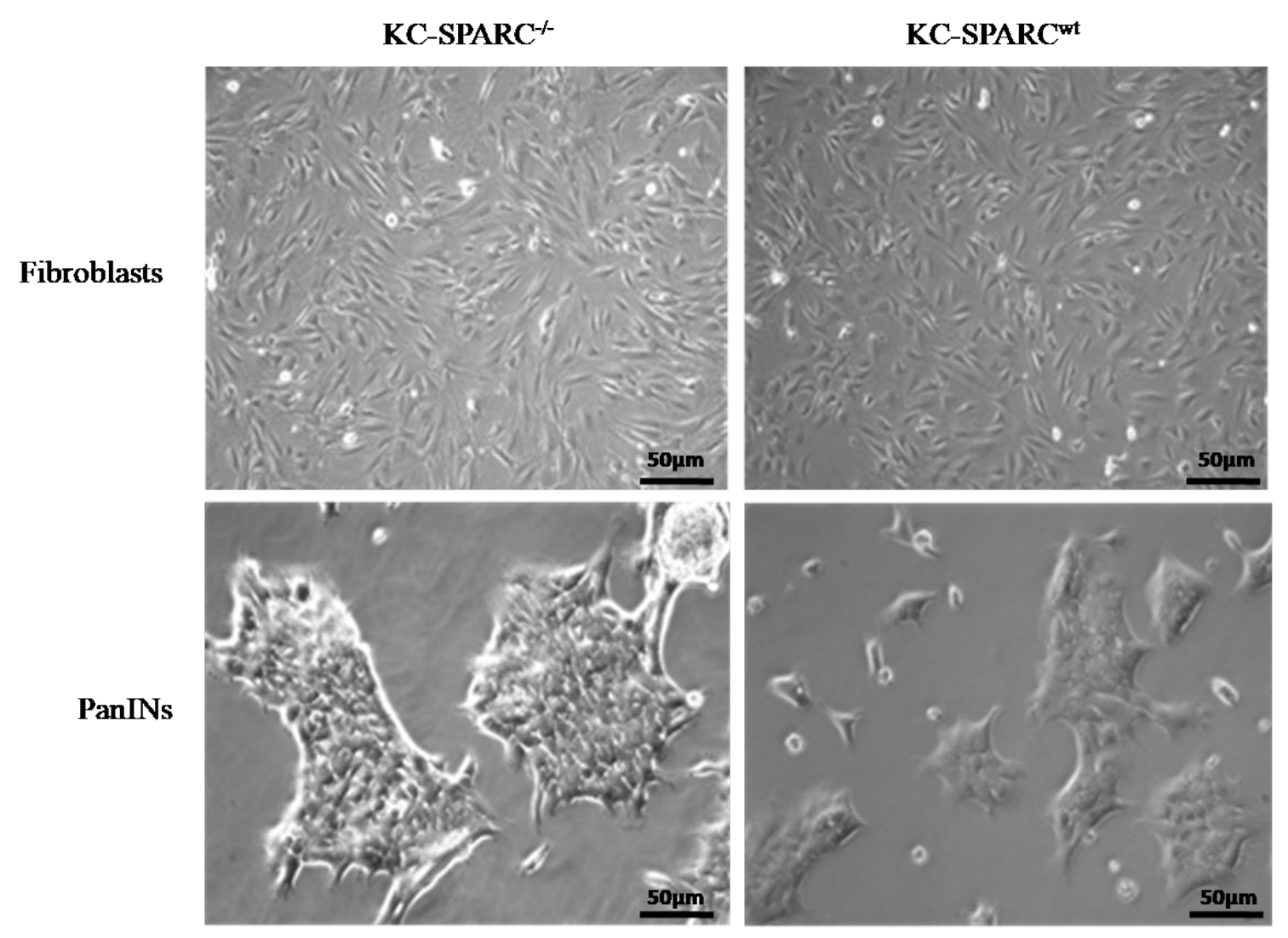

Figure 7: Representative bright field pictures of PAFs and PanINs cultures confirm the homogeneity of the population. Pictures were taken at 20x magnification. 


\subsubsection{Characterization of PAF cells by 1LoxP Kras genotype}

Having confirmed the homogeneity of the cultured PAF cells by microscopic observation, we further employed 1LoxP Kras PCR to ensure the purity of the culture at DNA level. In KC mice Cre mediated recombination results in one WT allele and one mutated allele with a LoxP site. 1LoxP Kras PCR specifically targets the single LoxP site left after the Cre recombination. PCR results showed clear bands at 285bp (WT allele) in the lanes of fibroblasts (no Cre expression) while in the positive control (DNA from PanIN cells) there are two bands at 285bp (WT allele) and 325bp (mutated allele with loxp-site). This confirms the purity of PAF cells at DNA level with no detectable contamination with PanIN cells.

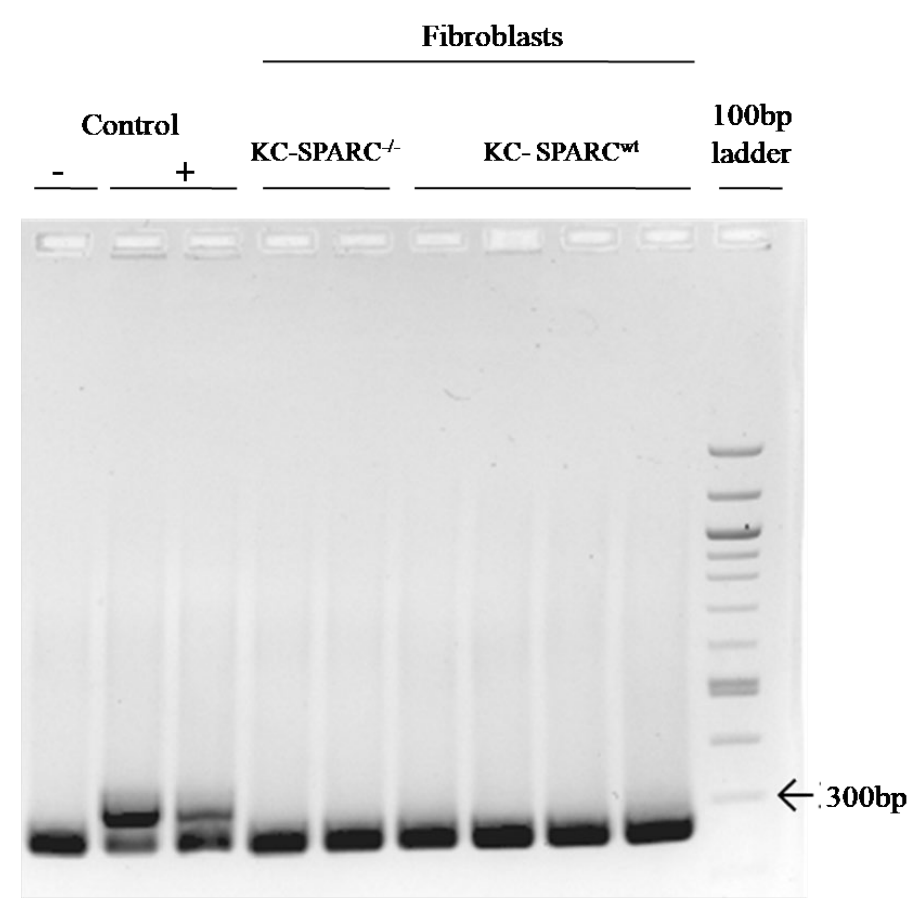

Figure 8: Agarose gel electrophoresis of 1LoxP Kras PCR product shows clear single band at 285bp (Kras WT allele) in fibroblast lanes. DNA from PanIN cells was used as a positive control showing a $285 \mathrm{bp}$ (WT allele) and $325 \mathrm{bp}$ (mutated allele with loxp site) as the result of Cre recombination. 100bp DNA ladder was used as a marker.

\subsubsection{Characterization of PanIN cells by western blot}

In order to further confirm the homogeneity of cultured primary PanIN cells, western blot analysis was performed for E-Cadherin (epithelial marker) and $\alpha$-SMA (fibroblast marker) in protein lysates of cultured PanIN cells. As shown in Fig. 9, E-Cadherin was strongly expressed by PanIN cells while there was no $\alpha$-SMA expression (except two clones) by PanIN cells. The slight bands for $\alpha$-SMA in two clones of KC-SPARC ${ }^{-/-}$ 
PanINs show that there is a contamination of fibroblasts. Therefore, those two clones were not used for further experiments.

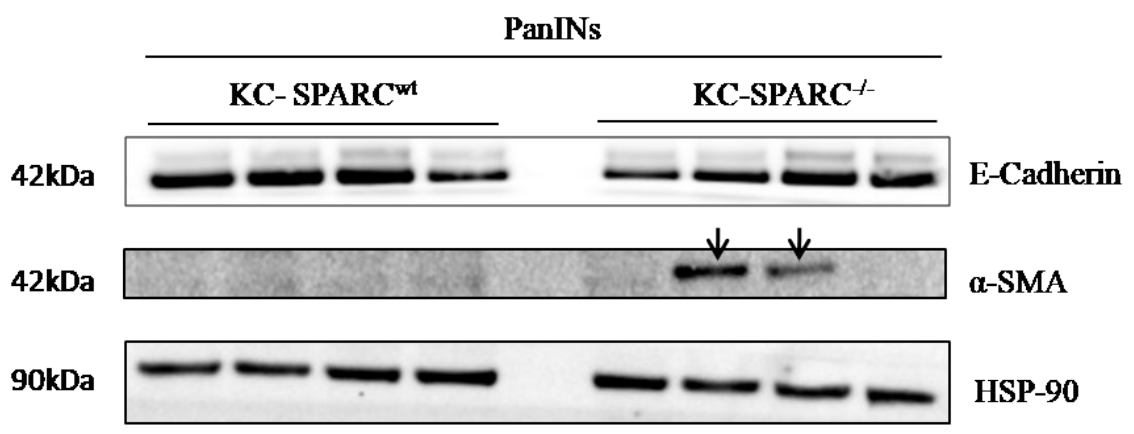

Figure 9: Western blot analysis shows strong expression of E-Cadherin by primary PanIN cells isolated from 3 months old $\mathrm{KC}^{-S P A R C}{ }^{\mathrm{wt}}$ and $\mathrm{KC}^{-S P A R C}{ }^{-/}$pancreata. In two clones of $\mathrm{KC}^{-S P A R C} \mathrm{C}^{-/}$PanINs (down arrow) there was expression of $\alpha$-SMA which shows contamination with fibroblasts. HSP-90 was used as a loading control.

\subsection{In vitro and in vivo expression of SPARC}

The expression of SPARC by isolated primary fibroblast and PanIN cells was assessed by western blot analysis. Furthermore, IHC for SPARC in KC-SPARC ${ }^{\text {wt }}$ and KC$\mathrm{SPARC}^{-/-}$mice pancreata with PanIN lesions and tumor was performed to investigate the in vivo expression of SPARC during different stages of PDAC progression. SPARC is not expressed in healthy pancreas. However, SPARC is overexpressed by CAFs during tumorigenesis of PDAC (Chen et al., 2010; Mantoni et al., 2008; Sato et al., 2003).

The western blot analysis performed in cell lysates of cultured primary fibroblasts (KCSPARC $^{\mathrm{wt}}$ and $\mathrm{KC}^{-S P A R C}{ }^{-/}$) and PanIN cells (KC-SPARC ${ }^{\mathrm{wt}}$ and $\mathrm{KC}^{-S P A R C}{ }^{-/}$) show strong expression of SPARC by fibroblasts of $\mathrm{KC}_{-S P A R C}{ }^{\mathrm{wt}}(\mathrm{n}=4)$, while there is nearly no expression in PanIN cells of KC-SPARC ${ }^{\mathrm{wt}}(\mathrm{n}=3)$. The lack of SPARC expression in fibroblasts $(n=3)$ and PanINs $(n=4)$ of $\mathrm{KC}-S P A R C^{-/-}$further confirms the genetic knock out of SPARC (Fig. 10A). Similarly, IHC analysis for SPARC in pre-neoplastic and tumor tissues of $\mathrm{KC}^{-S P A R C}{ }^{\mathrm{wt}}$ and $\mathrm{KC}-\mathrm{SPARC}^{-/-}$mice shows that there was no expression in the normal acinar cells and strong expression by PAFs and CAFs. Interestingly, SPARC is hardly expressed by tumor cells. The absence of positive staining in pre-neoplastic and tumor tissue of $\mathrm{KC}^{-S P A R C} \mathrm{~S}^{-/}$mice confirms the genetic ablation of SPARC in vivo (Fig. 10B). 
A.

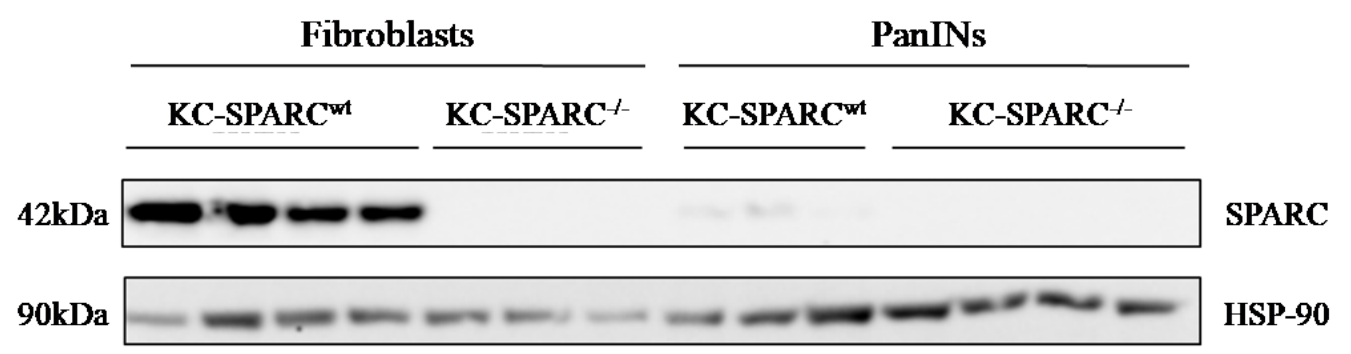

B.

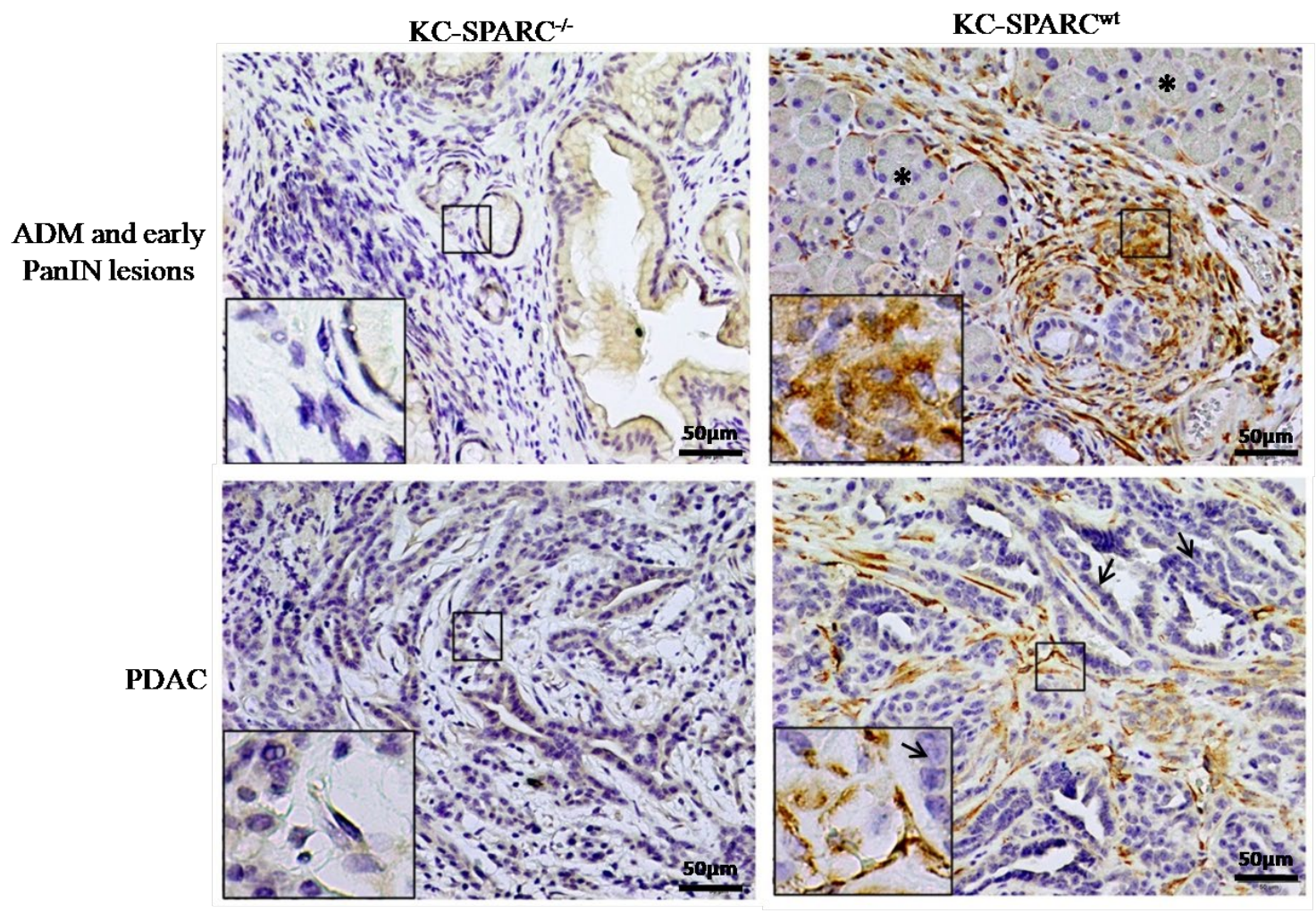

Figure 10: (A) Western blot analysis of cultured PAFs and PanIN cell lysates showing expression of SPARC in PAFs and hardly any expression in PanIN cells of KC-SPARC ${ }^{\text {wt }}$ mice. Absence of SPARC in PAFs and PanINs of KC-SPARC ${ }^{-/-}$confirms genetic knock out. HSP-90 was used as a loading control. (B) Representative pictures of IHC-SPARC in precursor lesions and PDAC of KC-SPARC ${ }^{\text {wt }}$ and $\mathrm{KC}^{-S P A R C} \mathrm{C}^{-}$ - shows that SPARC is not expressed in the normal acinar cells $\left(^{*}\right)$ and highly expressed by PAFs and CAFs of KC-SPARC ${ }^{\mathrm{wt}}$, while there is nearly no expression in PanIN and tumor cells (arrow). Images were taken at 20x magnification and the inset shows 40x magnification. 


\subsection{Early tumorigenesis}

\subsubsection{SPARC depletion does not affect ADMs, PanIN development and inflammation}

Having confirmed the elevated expression of SPARC during early tumorigenesis, we further elucidated whether ablation of SPARC promotes disease initiation and progression by performing a semi quantitative histological scoring of $\mathrm{H} \& \mathrm{E}$ stained pancreata from 3 months and 7-8 months old $\mathrm{KC}-S P A R C^{-/}$and $\mathrm{KC}^{-S P A R C}{ }^{\mathrm{wt}}$ mice. Histological scores from 1-3 were given based on the extent of ADMs and PanIN development as well as the immune cell infiltration. The scoring was performed by Prof. Dr. med. B. Sipos, Pathology, Universitätsklinikum, Tübingen. The semi-quantitative histological scoring shows that both $\mathrm{KC}-S P A R C^{\mathrm{wt}}$ and $\mathrm{KC}-\mathrm{SPARC}^{-/-}(3$ months and 7-8 months old) mice have comparable scoring of ADMs, PanIN1, PanIN2 and immune infiltration (Fig. 11A \&B). This finding indicates that expression of SPARC neither influences the disease initiation nor the disease progression in $\mathrm{KC}-\mathrm{SPARC}{ }^{\mathrm{wt}}$.

A.

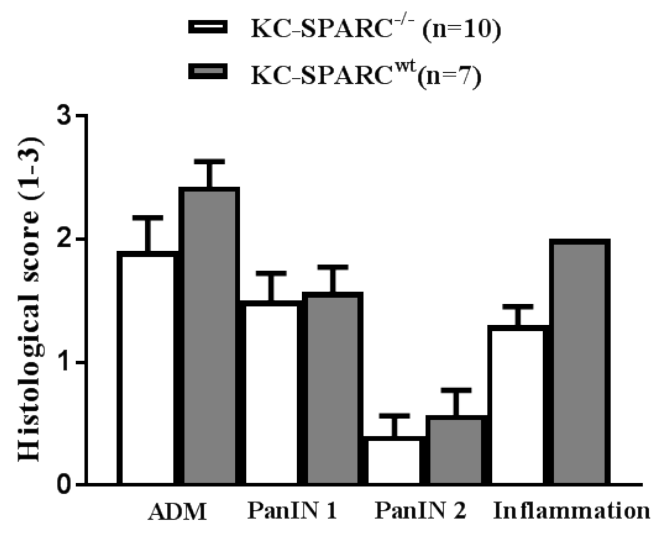

B.

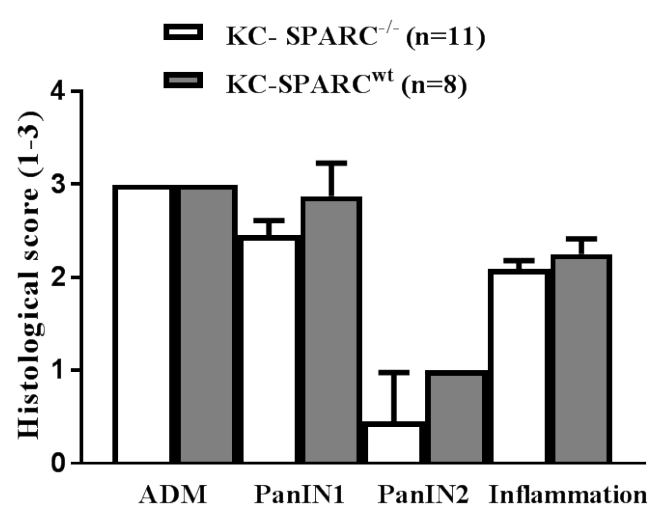

Figure 11: (A\&B) Semi-quantitative histological scoring of ADMs, PanIN1, PanIN2 and inflammation in

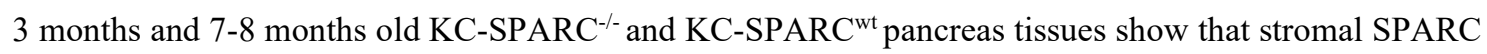
expression has no influence on disease progression. 


\subsubsection{Collagen deposition is significantly reduced upon SPARC depletion}

The abundant extra cellular matrix (e.g. collagen and hyaluronic acid) deposition has been reported to be associated with disease progression and poor clinical efficacy of drugs (Minchinton and Tannock, 2006; Netti et al., 2000). Furthermore, SPARC has been reported as a modulator of desmoplasia (Bradshaw, 2009; Tremble et al., 1993).

Therefore, to see if SPARC affects ECM deposition, two chemical stainings for collagen such as masson trichrome (total collagen) and Herovici (mature collagen) were performed in pre-neoplastic pancreatic tissues of 3 months old $\mathrm{KC}-S P A R C^{-/-}$and $\mathrm{KC}$ $\mathrm{SPARC}^{\mathrm{wt}}$ mice. Masson trichrome staining analysis of 3 months old pancreatic tissue revealed that the total collagen deposition was significantly reduced in pre-neoplastic pancreatic tissues of KC-SPARC ${ }^{-/}(n=10)$ mice compared to that of $\mathrm{KC}^{-S P A R C}{ }^{\mathrm{wt}}(\mathrm{n}=7)$ mice $(p=0.0001)$ (Fig 12A\&B).Masson trichrome staining analysis in 7-8 months old cohort (late tumorigenesis) exhibited similar trend though it did not reach statistical significance $(p=0.08)$ (Fig. 12C). These results show that depletion of SPARC affects collagen deposition regardless of disease progression stage, with the most pronounced effect during early PanIN development. Furthermore, herovici staining was performed to quantify the mature dense collagen deposition (Fig. 12D). Consistent with the total collagen deposition, the mature collagen deposition was also found to be reduced upon SPARC ablation. The mature dense collagen deposition was less in $\mathrm{KC}^{-S P A R C}{ }^{-/}$mice from both early ( 3 months) $(p=0.1)$ and late tumorigenesis $(7-8$ months $)(p=0.0002)$ cohort compared to that of KC-SPARC ${ }^{\mathrm{wt}}$ mice (Fig12 E\& F).

A.
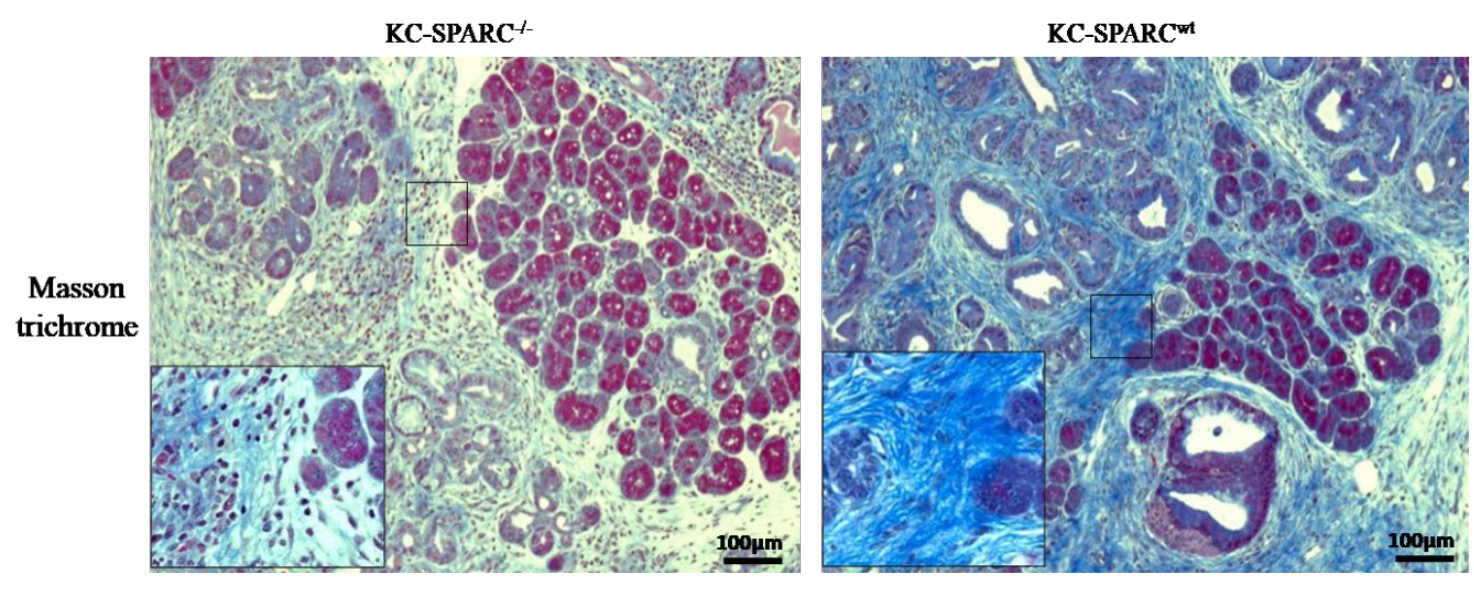
B.

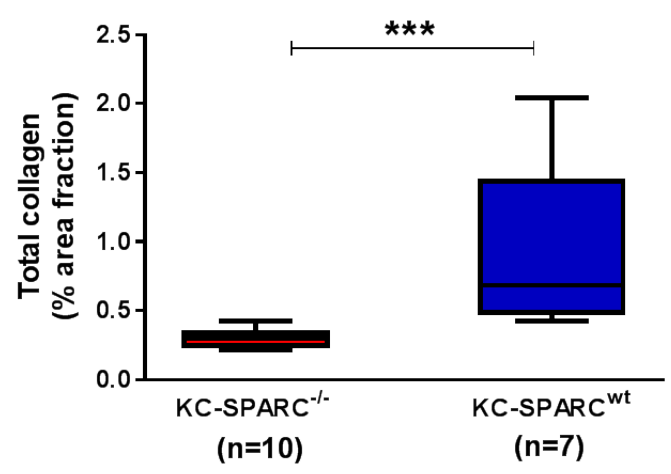

D.

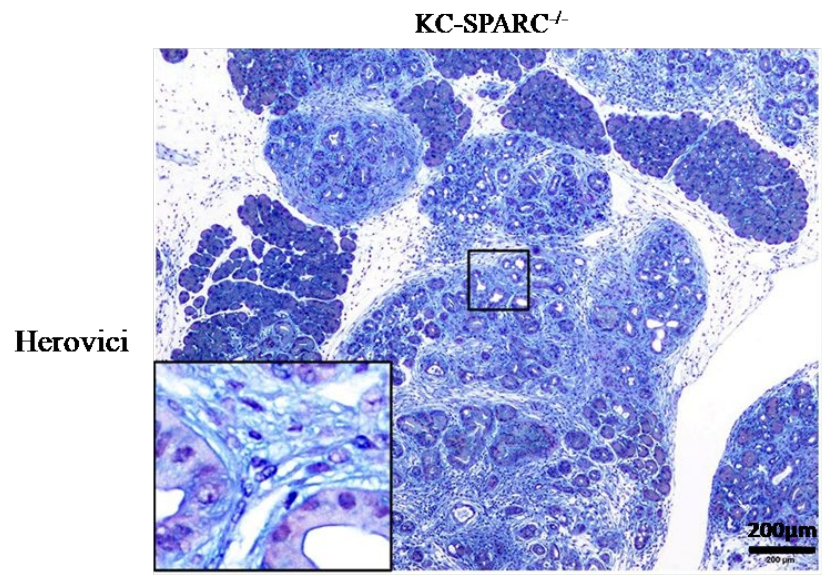

E.

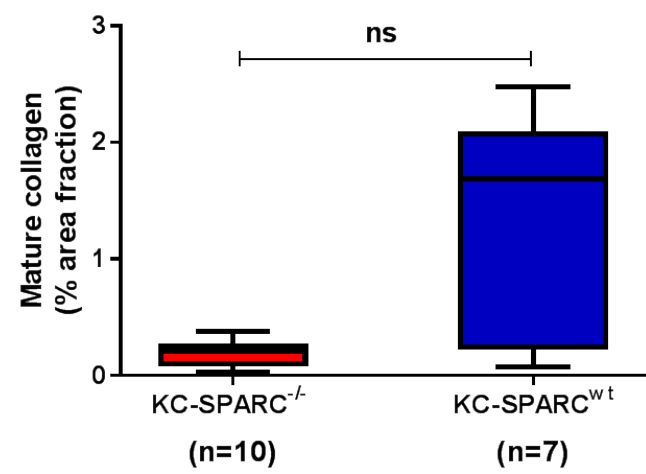

C.
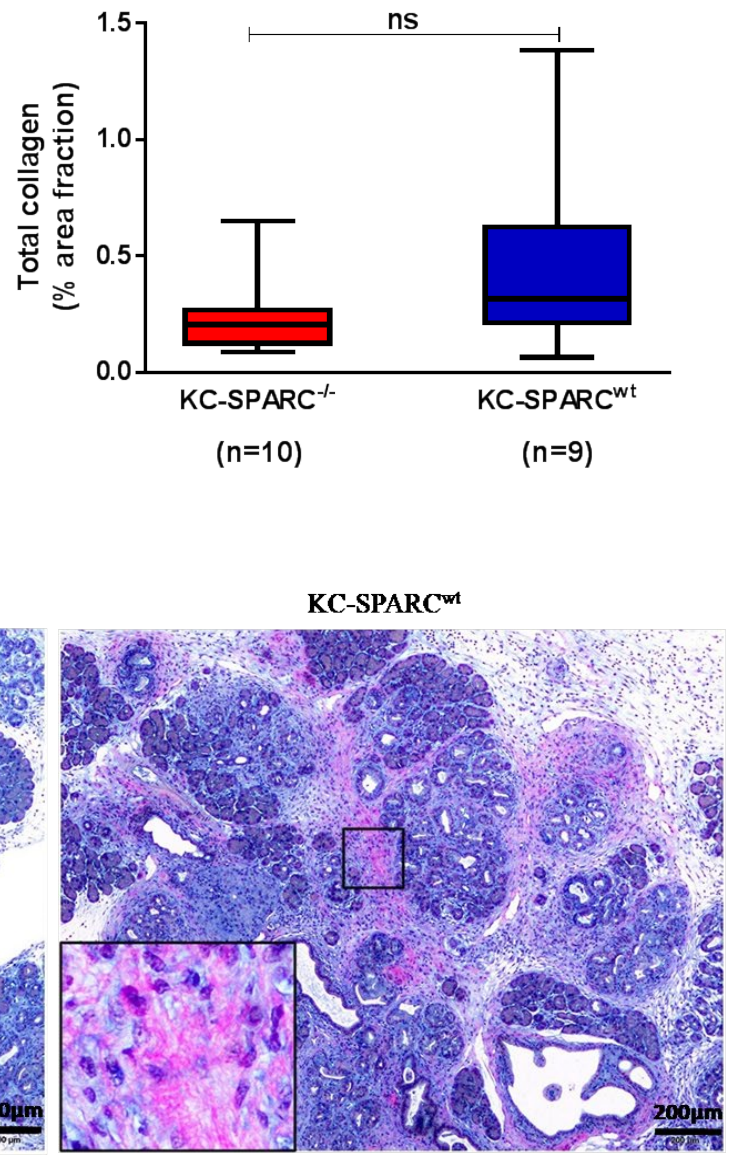

F.

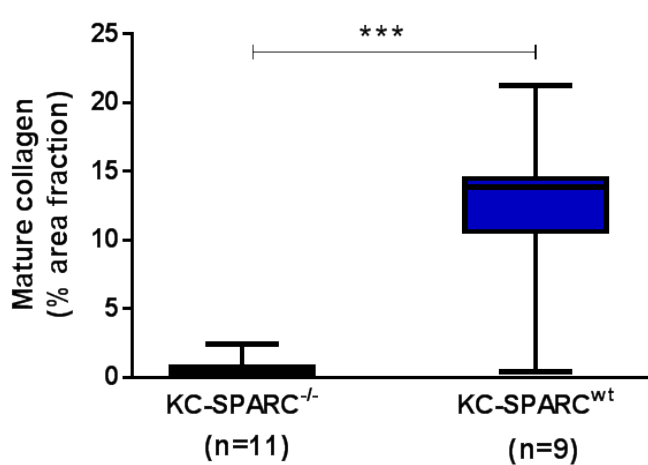

(B\&C) Quantification of masson trichrome staining in 3 months old $(\mathrm{p}=0.0001)$ and 7-8 months old $(\mathrm{p}=0.08) \mathrm{KC}^{-S P A R C}{ }^{-/}$and $\mathrm{KC}-\mathrm{SPARC}{ }^{\mathrm{wt}}$ mice pancreata. (D) Representative images of herovici staining in pancreas tissues of 3 months old $\mathrm{KC}^{-S P A R C^{-/}}$and $\mathrm{KC}-\mathrm{SPARC} \mathrm{C}^{\mathrm{wt}}$ mice. (E\&F) Quantification of herovici staining in 3 months old $(\mathrm{p}=0.11)$ and 7-8 months old $(\mathrm{p}=0.0002) \mathrm{KC}^{-S P A R C} \mathrm{C}^{-/}$and $\mathrm{KC}^{-S P A R C} \mathrm{Ct}^{\mathrm{wt}}$ mice pancreata. Statistical analysis was performed using Mann-Whitney test. $* * * \mathrm{p} \leq 0.005$. 


\subsubsection{SPARC depletion does not affect fibroblast population}

Activated PSCs are the major source of ECM components production (Apte et al., 1998; Bachem et al., 1998). Having confirmed the significant reduction of collagen deposition upon SPARC depletion, we assessed if depletion of SPARC directly affects the PSC population. To achieve this, IHC was performed for $\alpha$-SMA in pre-neoplastic pancreatic tissues of 3 months old KC-SPARC ${ }^{-/-}(n=9)$ and KC-SPARC ${ }^{w t}(n=6)$ mice. $\alpha$-SMA positive fibroblasts were specifically stained while the ductal and other cell population were devoid of positive staining as shown in Fig. 13A. The quantification of this staining was performed by measuring the $\alpha$-SMA positive area using color deconvolution tool, ImageJ software. The quantification of IHC- $\alpha$-SMA revealed that both $\mathrm{KC}-S P A R C^{-/}$and $\mathrm{KC}-S P A R C^{\mathrm{wt}}$ mice have comparable $\alpha$-SMA positive area $(4.16 \%$ vs $5.71 \%)(\mathrm{p}=0.38)$. This result shows that SPARC ablation does not affect the fibroblast population during early tumorigenesis.

A.
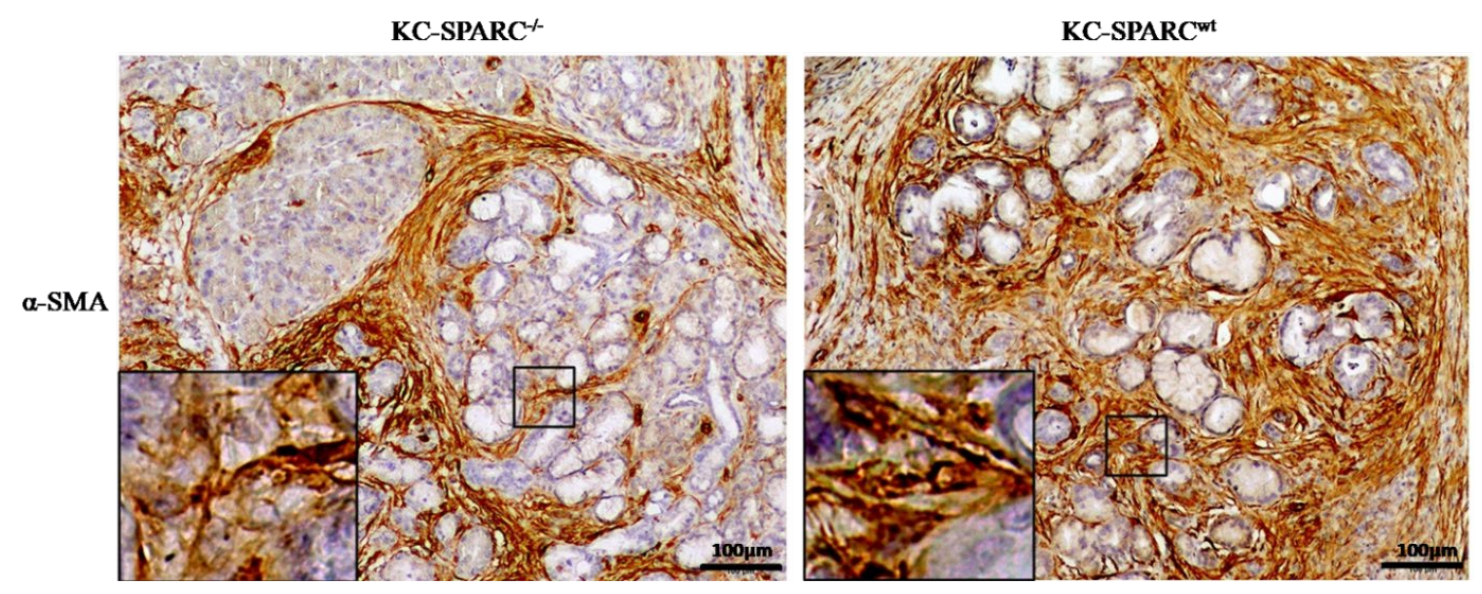

Figure 13: (A) Representative pictures of IHC- $\alpha$-SMA in pancreata of 3 months old KC-SPARC ${ }^{-1-} \mathrm{KC}-$ $\mathrm{SPARC}^{\mathrm{wt}}$ mice. Images were taken at $10 \mathrm{x}$ magnification, and the inset shows $40 \mathrm{x}$ magnification. 


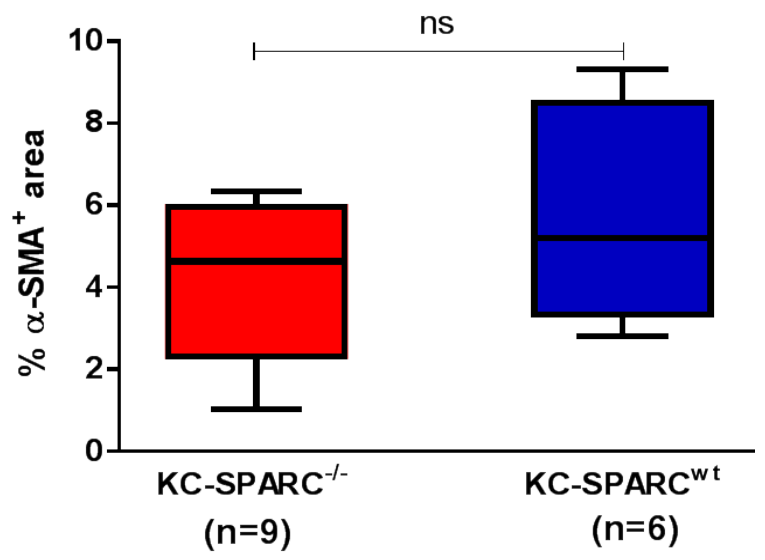

Figure 13: (B) Graph shows that KC- KC-SPARC ${ }^{-/}$and SPARC ${ }^{\mathrm{wt}}$ mice have comparable positive areas of $\alpha$-SMA ( $\mathrm{p}=0.38)$. Statistical analysis was performed using Mann-Whitney test.

\subsubsection{SPARC depletion does not affect overall proliferation in vivo}

The role of SPARC in proliferation has been reported in several tumor entities such as ovarian, melanoma, head and neck cancers. For instance, SPARC has been shown to act as key regulator of proliferation in ovarian cancer (Chen et al., 2012). In melanoma, SPARC seems to promote proliferation of stromal cells but not tumor cells in a context dependent manner (Haber et al., 2008). Therefore, to investigate whether SPARC depletion affects overall proliferation in vivo, IHC for Ki67was performed in preneoplastic pancreatic tissues ( 3 months old) in a SPARC dependent manner. Proliferation index ( $\%$ of proliferating cells) was calculated by dividing the number of $\mathrm{Ki} 67$ positive nuclei by total number of nuclei. The result was expressed in percentage. IHC- Ki67 specifically stained the nuclei of proliferating cells as shown in Fig. 14A. Quantification of the staining revealed that SPARC status does not affect the overall proliferation of preneoplastic lesions as PanIN lesions of both KC-SPARC ${ }^{-/-}(\mathrm{n}=10)$ and KC-SPARC ${ }^{\mathrm{wt}}(\mathrm{n}=7)$ mice have similar proliferation rate $(5 \mathrm{vs} 6 \%$ proliferating nuclei) $(\mathrm{p}=0.08)$. 
A.

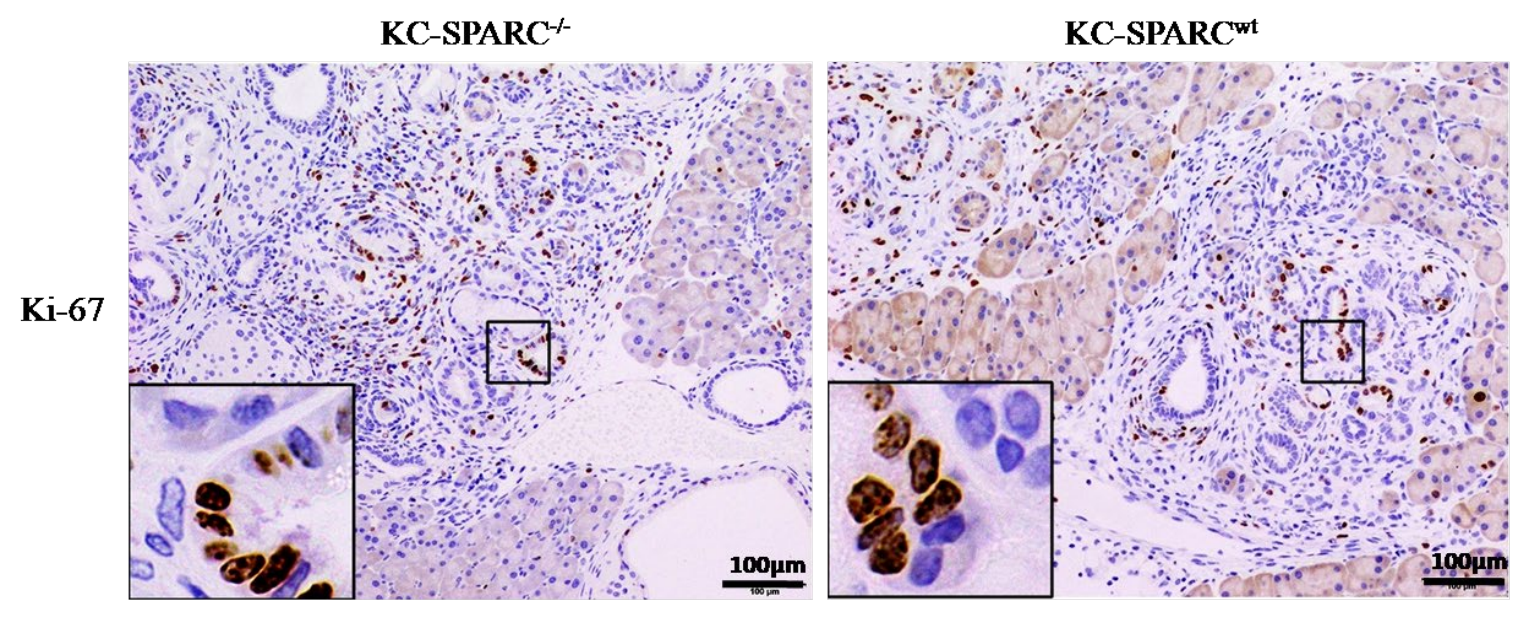

B.

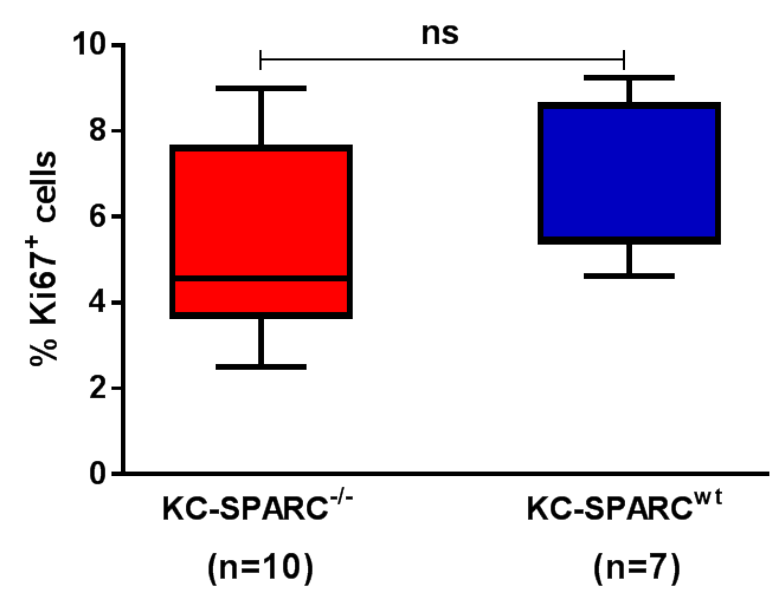

Figure 14: (A) Representative pictures of IHC-Ki67 in the PanIN lesions of KC-SPARC ${ }^{-/}(n=10)$ and KC$\operatorname{SPARC}^{\mathrm{wt}}(\mathrm{n}=7)$ mice. Images were taken at 10x magnification and the inset shows 40x magnification. (B) Graph shows that SPARC ablation did not affect the overall proliferation the PanIN lesions $(\mathrm{p}=0.08)$. Statistical analysis was performed using Mann-Whitney test. 


\subsubsection{Stroma derived SPARC does not affect migration of PanIN cells}

The role SPARC on tumor cell migration and invasion has been investigated in many tumor entities and was reported to play pro- and anti-invasive role in different cancers. Overexpression of SPARC has been shown to increase the risk of metastasis in various tumors such as melanoma, glioblastoma, clear-cell renal carcinoma, prostate carcinoma, breast and pancreatic ductal adenocarcinoma (Arnold and Brekken, 2009; Arnold et al., 2012; Nagaraju et al., 2014).

To investigate whether SPARC influences the migration of cells in vitro, PanIN and

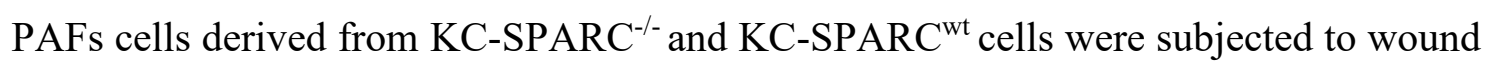
healing assays without any exogenous stimulation. Wound healing assays were performed with KC-SPARC ${ }^{-/-}(n=3)$ and KC-SPARC ${ }^{\mathrm{wt}}(\mathrm{n}=3)$ PAF cells showed no difference in terms of migration regardless of SPARC status (Fig. 15A). Similar results were obtained in wound healing assays performed with PanIN cells (Fig 15B). However, SPARC is predominantly secreted by activated fibroblast while there is almost no protein expression in the epithelial compartment as shown in Fig. 10. Therefore, to investigate if exogenous SPARC has any effect on wound healing properties of $\mathrm{KC}-\mathrm{SPARC}^{-/}$PanIN cells, we performed wound healing assay upon stimulation with the conditioned media (culture supernatant) prepared from $\mathrm{KC}-\mathrm{SPARC}^{-/}$and $\mathrm{KC}^{-S P A R C}{ }^{\mathrm{wt}} \mathrm{PAFs}$. Result show that stimulation with PAF conditioned media did not influence the wound healing properties of $\mathrm{KC}-S P A R C^{-/-}$PanIN cells (Fig. 15C). 
4. Results

A.
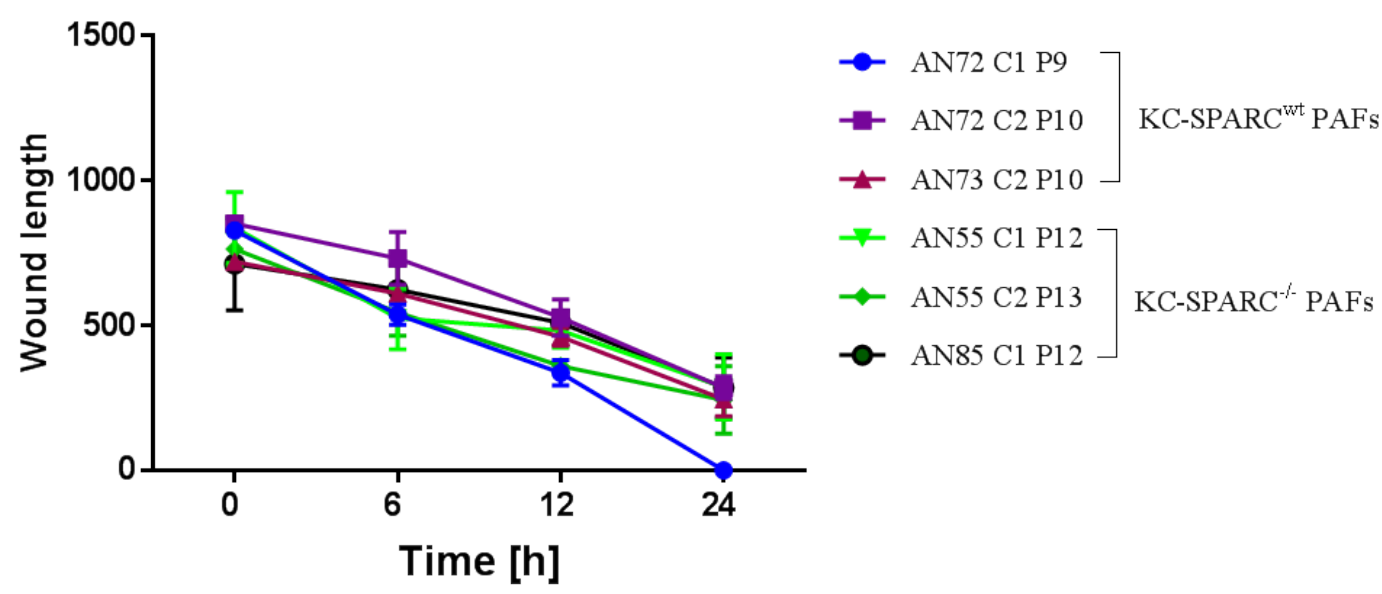

B.

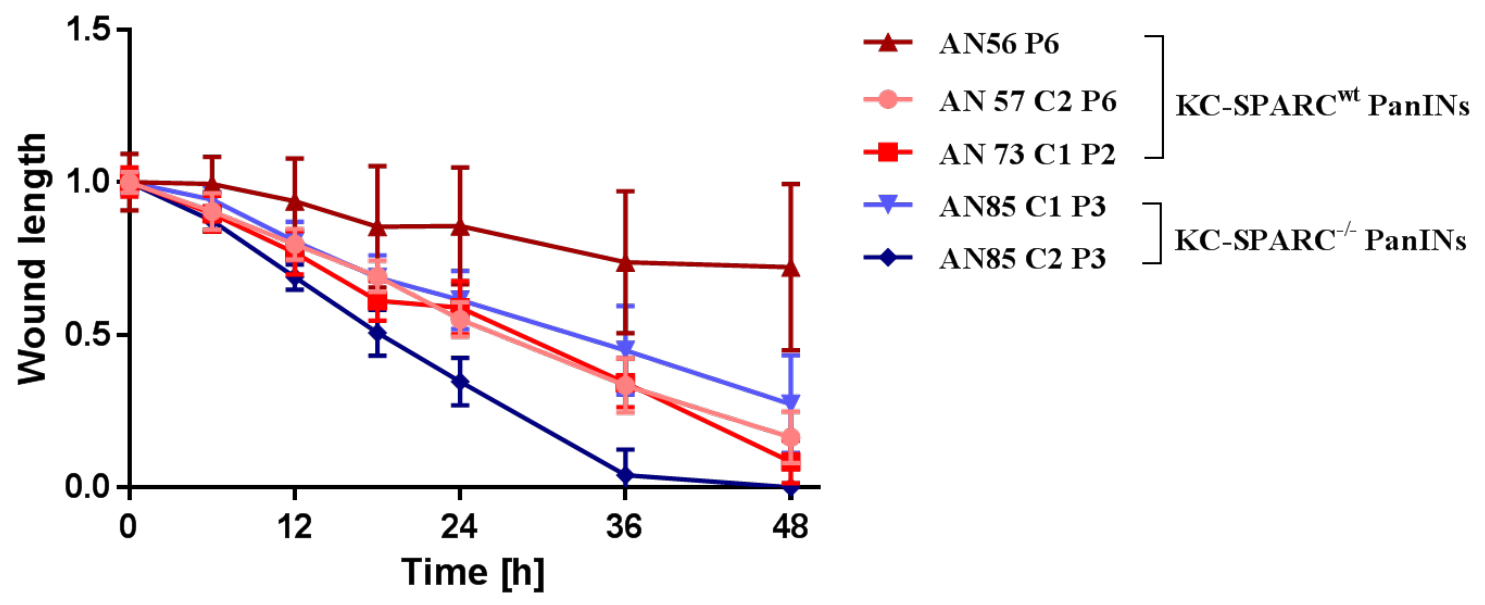

C.

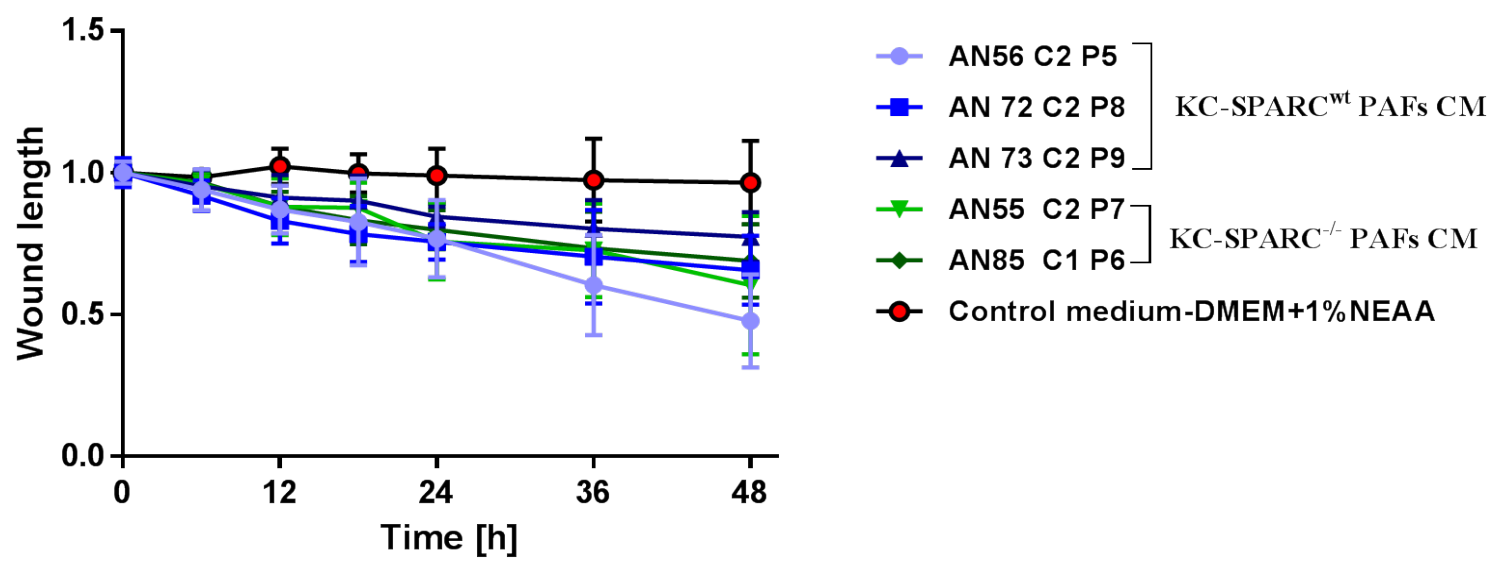

Figure 15: Wound healing assays performed in isolated primary (A) PAF cells and (B) PanIN cells from $\mathrm{KC}^{-S P A R C}{ }^{\mathrm{wt}}$ and $\mathrm{KC}^{-S P A R C} \mathrm{C}^{-/}$mice shows that SPARC depletion does not affect the migration of both cell types in 2D based wound healing assays. (C) PanIN cells from KC-SPARC ${ }^{-/-}$were treated with conditioned media from PAFs derived from KC-SPARC ${ }^{\mathrm{wt}}$ and $\mathrm{KC}^{-S P A R C}{ }^{-/-}$mice and wound healing assays were performed showing no difference upon stromal derived SPARC. 


\subsubsection{SPARC and angiogenesis during early and late tumorigenesis}

Angiogenesis is the process of forming blood vessels and it is important for tumor growth and to enhance metastatic spread. Angiogenesis is achieved through proliferation of endothelial cells. SPARC has been shown to play an anti-angiogenic role in various tumor entities such as gastric cancer and neuroblastoma (Chlenski et al., 2010; Zhang et al., 2012). To investigate whether stromal derived SPARC plays a pro- or anti-angiogenic role during tumorigenesis, immunohistochemistry was performed for CD31 in 3-4 months and 7-8 months old KC-SPARC ${ }^{-/-}(\mathrm{n}=10$ and 11$)$ and $\mathrm{KC}-S P A R C^{\mathrm{wt}}(\mathrm{n}=7$ and 9) pancreata bearing early and advanced PanIN lesions respectively. Interestingly, there was no significant difference between $\mathrm{KC}^{-S P A R C} \mathrm{wt}^{\mathrm{wt}}$ and $\mathrm{KC}^{-S P A R C} \mathrm{C}^{-/}$mice regarding mean vessel density in both the 3 months old cohort $(p=0.66)$ and the 7-8 months old cohort $(\mathrm{p}=0.15)$.

A.
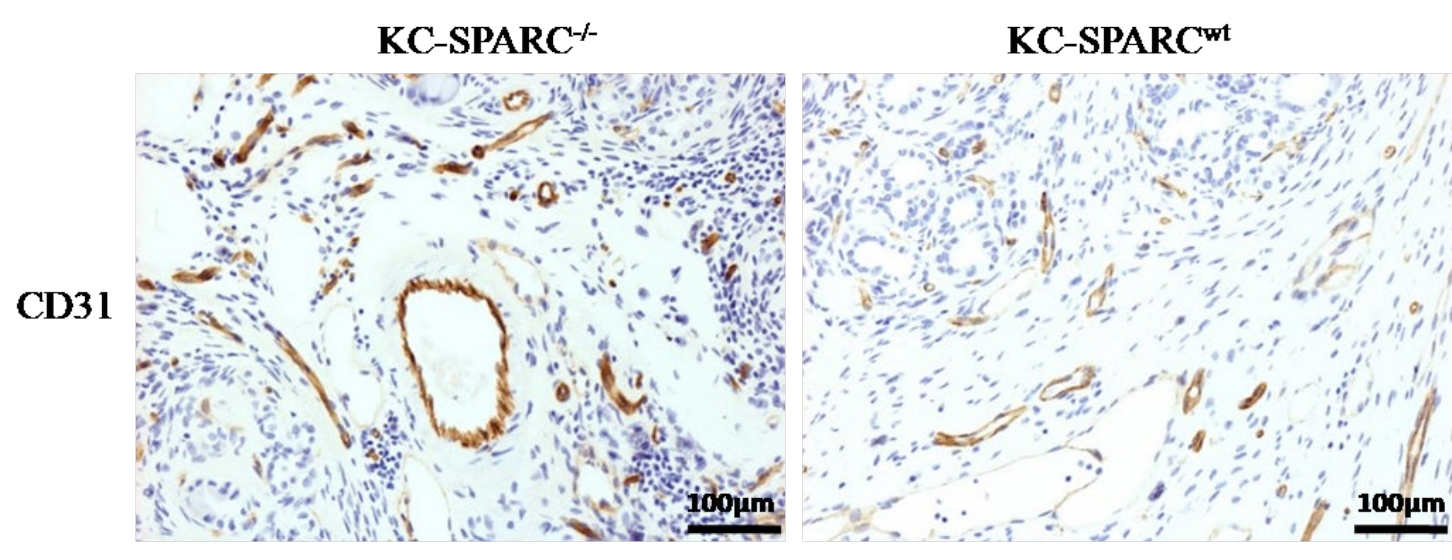

B.

C.
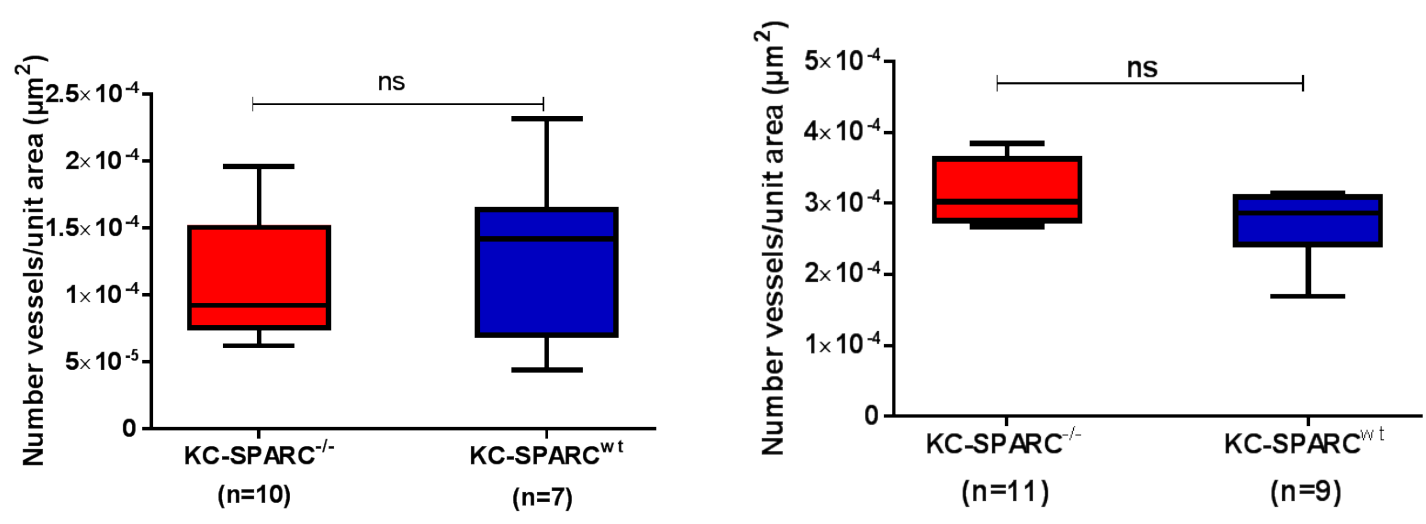

Figure 16: (A) Representative pictures of IHC-CD-31 in 3 months old pancreas tissues of KC-SPARC ${ }^{\text {wt }}$ and $\mathrm{KC}$-SPARC ${ }^{-/}$mice taken at10x magnification. (B\&C) Graphs show no statistical significance in terms 
of number of vessels between 3 months and 7-8 months old $\mathrm{KC}^{-S P A R C}{ }^{\mathrm{wt}}$ and $\mathrm{KC}$-SPARC ${ }^{-/}$mice $(\mathrm{p}=0.66)$ $(p=0.15)$ respectively. Statistical analysis was performed using Mann-Whitney test.

\subsection{SPARC in invasive adenocarcinoma}

\subsubsection{SPARC status does not affect the tumor incidence and the tumor weight}

Both $\mathrm{KC}-S P A R C^{-/-}$and $\mathrm{KC}^{-S P A R C} \mathrm{Wt}^{\mathrm{wt}}$ mice developed invasive adenocarcinoma at about 12 or more months. The tumor incidence was calculated by dividing the number of mice that developed tumors by the total number of mice in the cohort. Tumor incidence analysis (Fig. 17A) revealed that both $\mathrm{KC}-S P A R C^{-/}$and $\mathrm{KC}-S P A R C^{\text {wt }}$ cohorts have comparable tumor incidences. In the $\mathrm{KC}-S P A R C^{-/-}$cohort 26 mice out of 53 mice (49\%) developed tumors while in the SPARC ${ }^{\mathrm{wt}}$ cohort 19 mice out of 29 mice developed tumors $(66 \%)$. Chi-square (Fisher's exact test) was used to assess the statistical significance $(\mathrm{p}=0.17)$. The weight of the whole tumors was recorded during the sample collection and the relative tumor weight was calculated by normalizing the tumor weight with body weight. The relative tumor weight analysis (Fig. 17B) showed that the tumors of KCSPARC $^{-/-}(n=15)$ and KC-SPARC ${ }^{\text {wt }}(n=11)$ were of similar size $(p=0.75)$. Mann Whitney test was used for statistical analysis.

A.

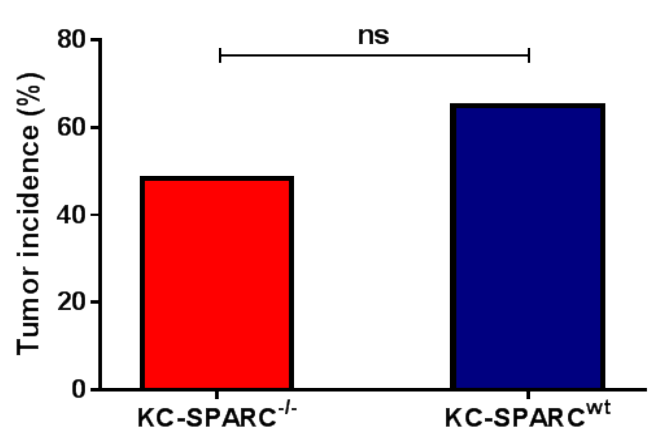

(26 out of 53 mice) (19 out of 29 mice)
B.

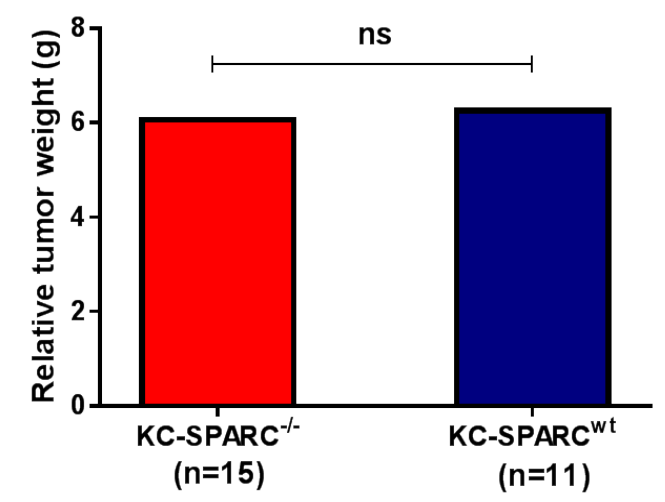

Figure: (A) Graph of tumor incidence in KC-SPARC ${ }^{--}(49 \%)$ and $\mathrm{KC}^{-S P A R C}{ }^{\mathrm{wt}}(66 \%)$ cohort. Statistical analysis was performed using Chi-square (Fisher's exact test) $(\mathrm{p}=0.17$ ). (B) Graph of relative tumor weight

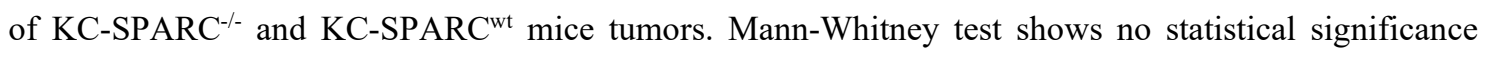
$(\mathrm{p}=0.75)$. 


\section{Results}

\subsubsection{Collagen deposition was impaired upon SPARC depletion in established tumors}

To explore the effect of SPARC depletion on collagen deposition in established murine pancreatic tumors we performed chemical stainings for collagen such as masson trichrome and picrosirius in tumor tissues of KC-SPARC ${ }^{\mathrm{wt}}(\mathrm{n} \geq 7)$ and $\mathrm{KC}^{-S_{P A R C}}{ }^{-/}$ $(\mathrm{n} \geq 17)$ mice. Consistent with the results in PanIN lesions (Figure 12), loss of SPARC resulted in a significant reduction of collagen deposition in established tumors (Fig. 20A). Quantification of masson trichrome and picrosirius staining was performed using an area based analysis in ImageJ software. The results of masson trichrome and picrosirius were expressed as $\%$ area fraction and $\%$ of picrosirius red positive area, respectively. Quantification of masson trichrome staining in tumor tissues of KC-SPARC ${ }^{-/-}(13 \%)$ and $\mathrm{KC}-\mathrm{SPARC}^{\mathrm{wt}}(22 \%)$ mice revealed that the total collagen deposition was significantly reduced in ${\mathrm{KC}-S P A R C^{-/}}^{-}$tumors $(\mathrm{p}=0.04)$ (Fig. 20B). The significant reduction in collagen deposition was further confirmed by picrosirius staining analysis $(\mathrm{p}=0.01)$ (Fig. 20C).

A.

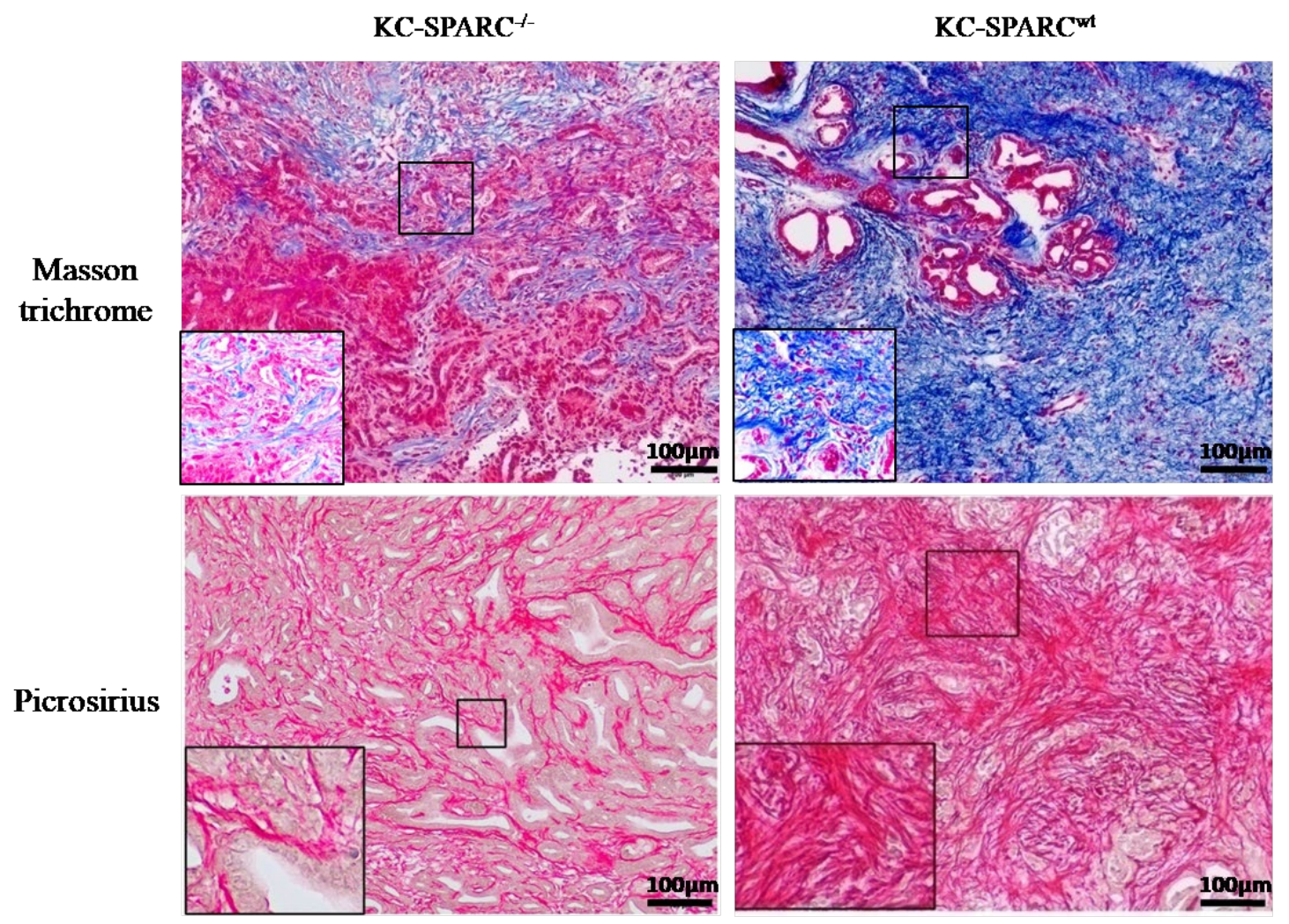

Figure 20: (A) Representative pictures of masson trichrome and picrosirius red stain in established tumor tissues of ${\mathrm{KC}-S P A R C^{-/}}^{-}$and $\mathrm{KC}^{-S P A R C} \mathrm{C}^{\mathrm{wt}}$ mice. The images were taken at $10 \mathrm{x}$ magnification (inset $40 \mathrm{x}$ magnification). 
B.

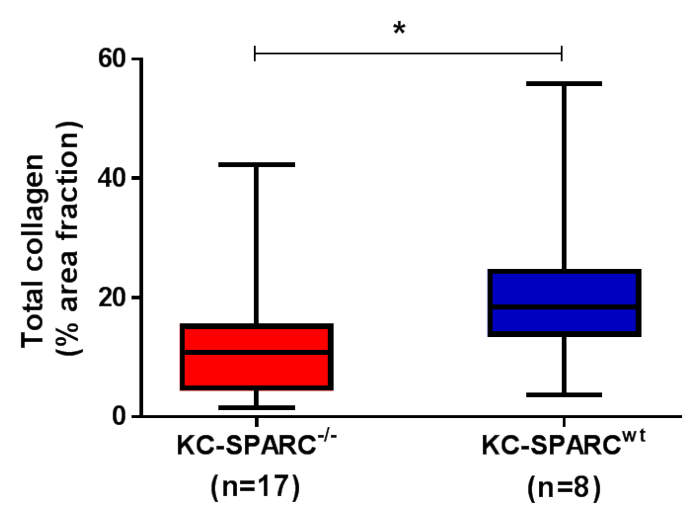

C.

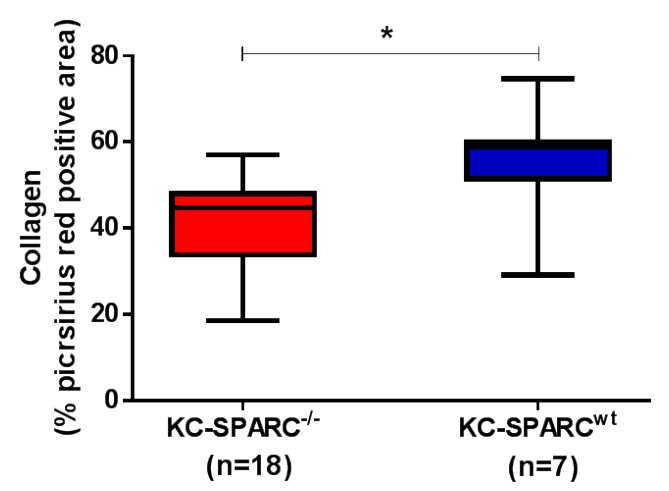

(B) Quantification of masson trichrome staining $(\mathrm{p}=0.04)$ and picrosirius red $(\mathrm{p}=0.01)$ shows significant reduction of collagen deposition in established tumors of $\mathrm{KC}-S P A R C^{-/}$mice. Mann-Whitney test was used to assess the statistical significance. ${ }^{*} \mathrm{p} \leq 0.05$.

\subsubsection{SPARC and hyaluronic acid deposition in tumor}

HA is an abundant ECM component which has been shown to modulate the tumor microenvironment. HA is overexpressed in many tumors and the amount of HA is directly correlated with a poor prognosis in patients (Chanmee et al., 2016). The significance of HA expression and deposition has been described in many tumor entities such as breast, bladder and pancreatic cancer (Mahlbacher et al., 1992)(Lokeshwar et al., 1997; Yahya et al., 2014). In pancreatic cancer, HA has been shown to promote tumor growth (Sato et al., 2003, 2016).

Therefore, to investigate whether SPARC affects HA deposition in established KC tumors, chemical staining for HA in established tumors of KC-SPARC $\mathrm{K}^{-/-}(\mathrm{n}=17)$ and $\mathrm{KC}$ SPARC $^{\mathrm{wt}}(\mathrm{n}=8)$ mice was performed. Quantification of HA staining showed similar amount of accumulation of HA in the tumors of $\mathrm{KC}-S P A R C^{-/-}$and $\mathrm{KC}-S P A R C^{\mathrm{wt}}$ mice $(p=0.97)$ and thus revealed that SPARC status does not affect HA accumulation in established tumors (Fig. 21A \& B). 
A.

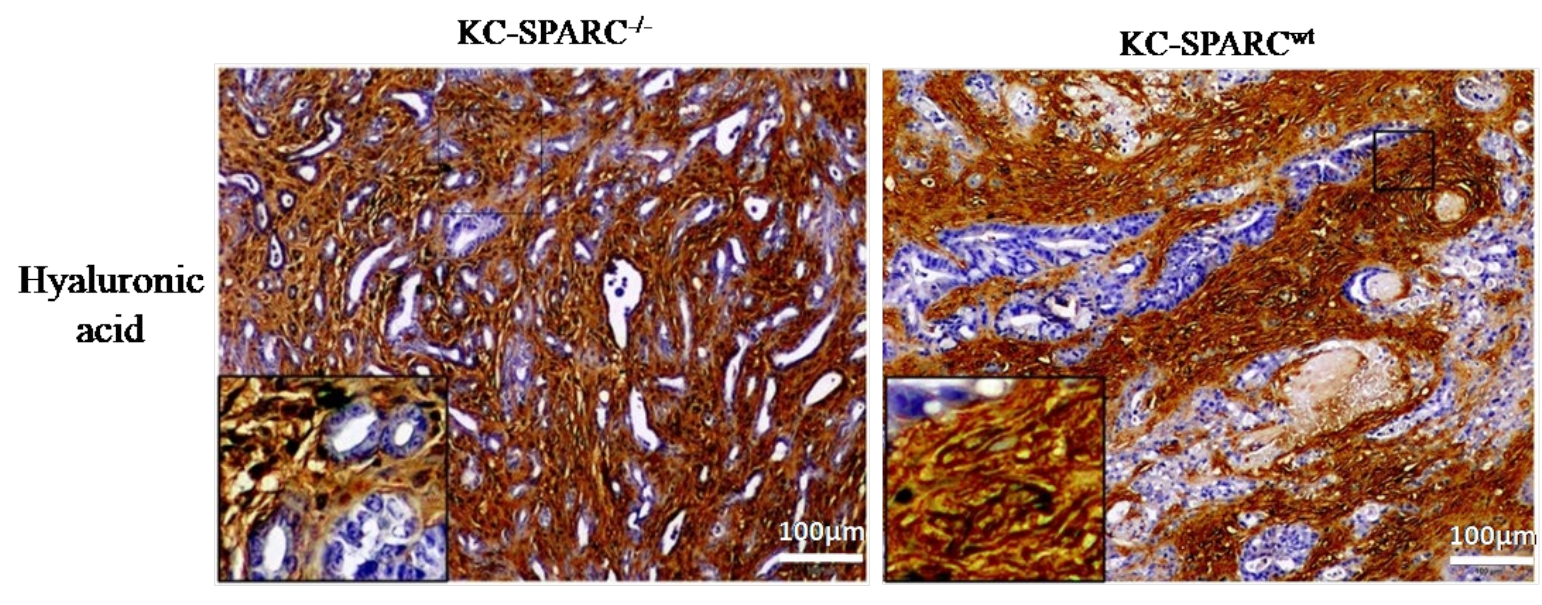

B.

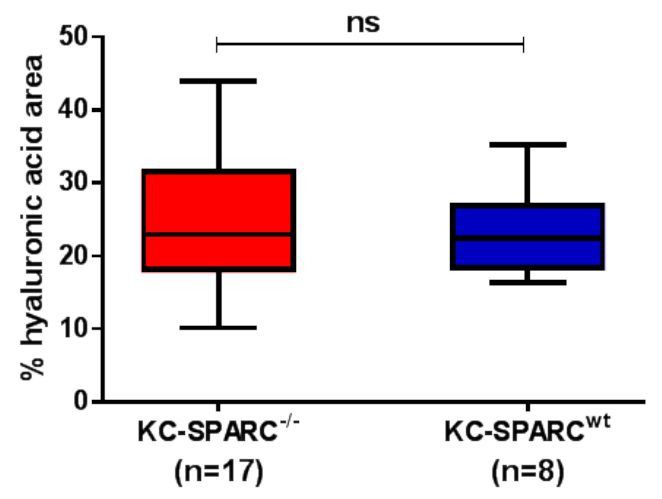

Figure 21: (A) Representative pictures of HA stainings in $\mathrm{KC}-\mathrm{SPARC}^{-/-}$and $\mathrm{KC}-\mathrm{SPARC}^{\mathrm{wt}}$ tumors. Both ${\mathrm{KC}-S P A R C^{-/-}}$and KC-SPARC ${ }^{\mathrm{wt}}$ tumors show extensive accumulation of HA. (B) Quantification of HA staining shows that SPARC depletion does not affect HA accumulation in tumors ( $p=0.97)$.Mann-Whitney test shows no statistical significance.

\subsubsection{SPARC depletion does not affect fibroblast density in established tumor}

As shown in the early tumorigenesis cohort, we investigated if SPARC affected the fibroblast population in established tumor by performing IHC for $\alpha$-SMA in the tumor tissue sections of KC-SPARC ${ }^{-/}(\mathrm{n}=16)$ and $\mathrm{KC}^{-S P A R C}{ }^{\mathrm{wt}}(\mathrm{n}=8)$ mice. Consistent with the results shown in the early PanIN cohort (Fig. 13A \&B), the fibroblast density was not altered in established tumors upon SPARC depletion (16\% vs $15 \% \mathrm{KC}-\mathrm{SPARC}^{-/}$and $\mathrm{KC}$ SPARC $^{\text {wt }}$ tumors) $(\mathrm{p}=0.78)$ (Fig. 22). 


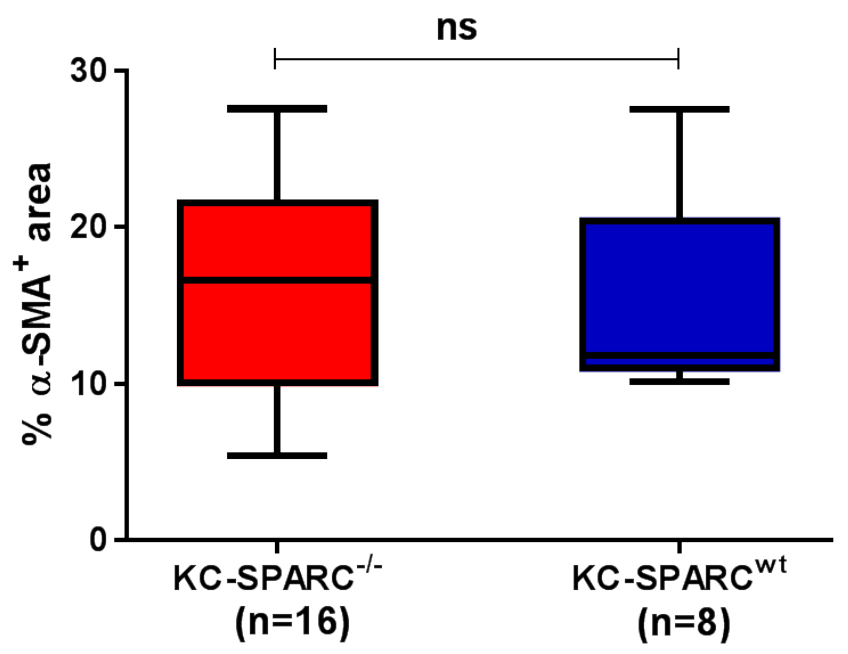

Figure 22: Graph shows that $\alpha$-SMA positive fibroblast density was not altered upon SPARC depletion $(p=0.78)$. Mann-Whitney test shows no statistical significance.

\subsubsection{Tumor proliferation and apoptosis were not affected by stromal SPARC}

Uncontrolled proliferation and decreased apoptosis are the hallmark features of tumors. An increased number of proliferating cells and decreased rate of cell death result in tumor growth and aggressiveness. To assess the proliferation index of tumors in $\mathrm{KC}-\mathrm{SPARC}^{-/-}$ $(\mathrm{n}=6)$ and KC-SPARC ${ }^{\mathrm{wt}}(\mathrm{n}=7)$ mice, IHC for Ki67 was performed (Fig 23A-top images). The proliferation index was determined by dividing the number of Ki67 positive nuclei by the total number of nuclei and was expressed as \% of Ki67 positive cells. To assess the apoptotic rate of the tumor, IHC for Cleaved Caspase 3 (CC3) was performed in tumor tissues of KC-SPARC ${ }^{-/-}(n=12)$ and KC-SPARC ${ }^{\mathrm{wt}}(\mathrm{n}=7)$ mice (Fig 23A-bottom images). Percentage of apoptotic nuclei was assessed using similar formula as mentioned above.

Analysis of IHC-Ki67 shows that both $\mathrm{KC}-\mathrm{SPARC}^{-/-}$and $\mathrm{KC}-\mathrm{SPARC}{ }^{\mathrm{wt}}$ tumors have similar proliferation index and thus revealed that stromal SPARC does not affect overall proliferation (Fig. 23B). The mean proliferation index of $\mathrm{KC}-S P A R C^{-/-}$and $\mathrm{KC}$ SPARC ${ }^{\mathrm{wt}}$ tumors were $24 \%$ and $29 \%$ respectively $(\mathrm{p}=0.44)$. In line with the proliferation index, IHC-CC3 analysis showed no difference in apoptotic cells between the tumors of $\mathrm{KC}-S P A R C^{-/-}$and $\mathrm{KC}^{-S P A R C}{ }^{\mathrm{wt}}$ mice $(0.82 \%$ vs $0.86 \%$; $\mathrm{p}=0.85)$ (Fig. 23C). 
A.
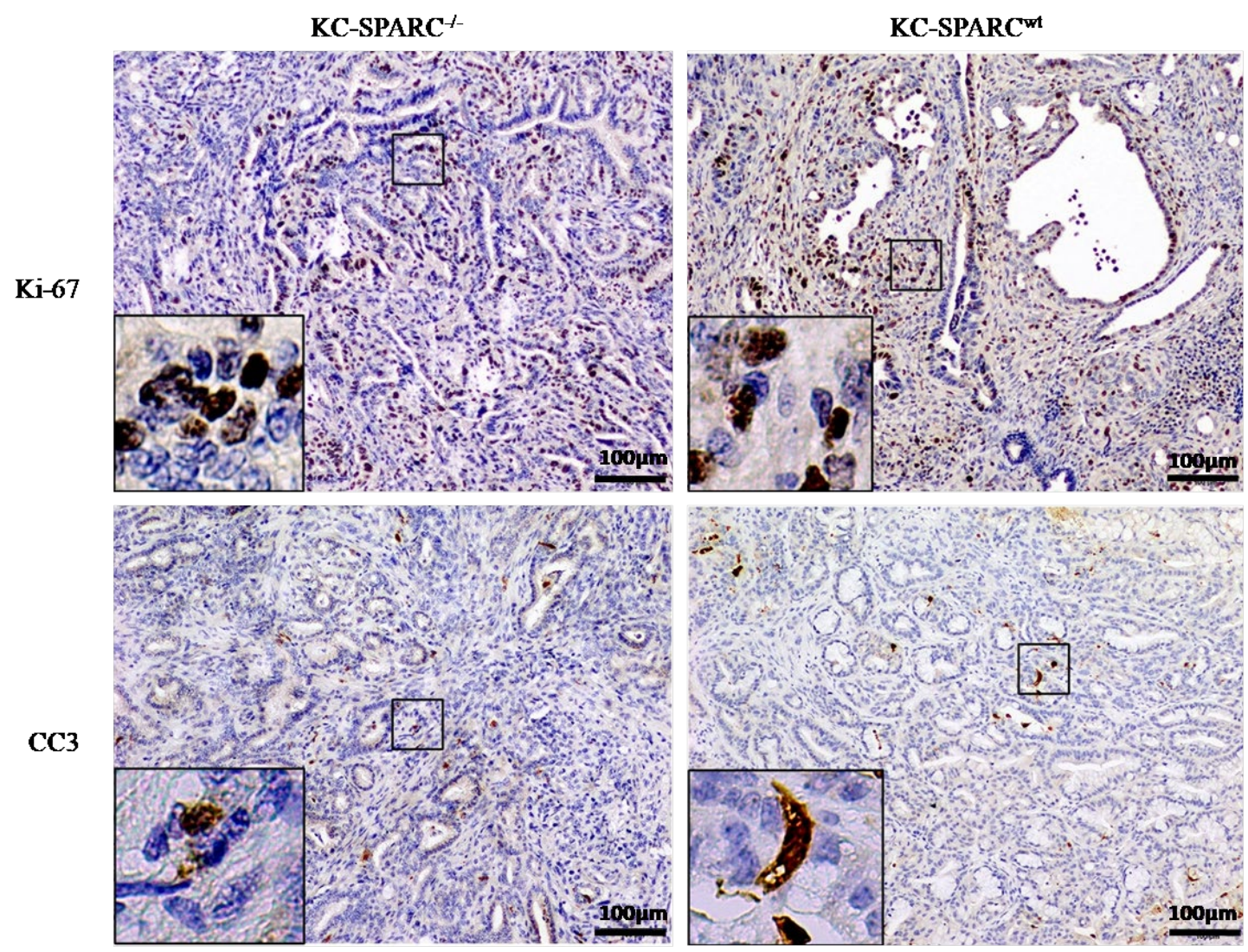

B.

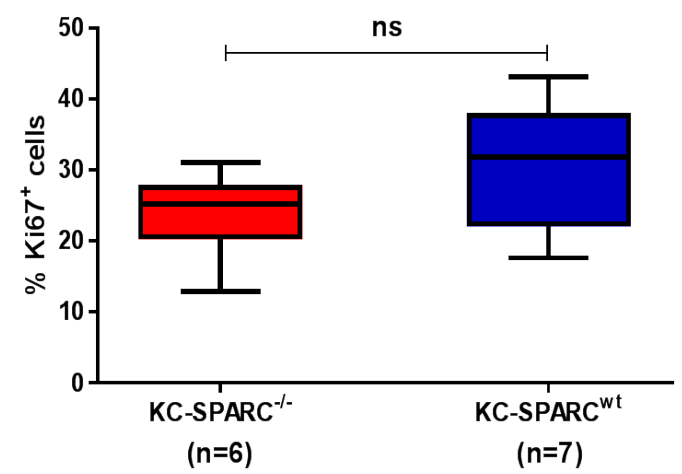

C.

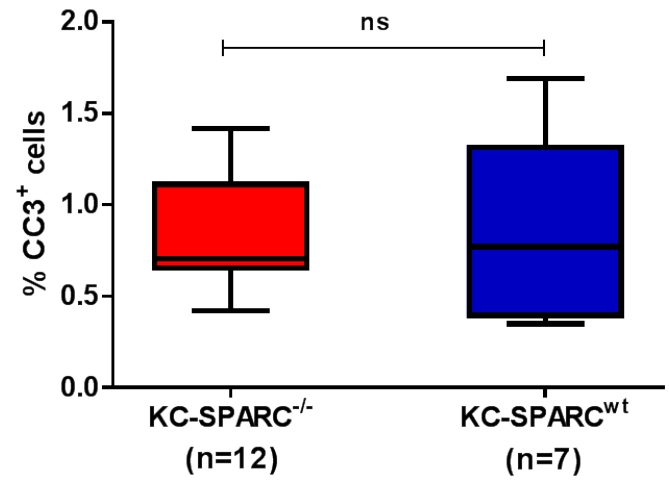

Figure 23: Representative pictures of IHC-Ki-67 and IHC-CC3 in KC-SPARC ${ }^{\text {wt }}$ and KC-SPARC ${ }^{-/}$tumors. (B) Quantification of IHC-Ki67 ( $\mathrm{p}=0.44)$ and C) IHC-CC3 $(\mathrm{p}=0.85)$ shows that proliferation and apoptosis was not affected by stromal SPARC expression.

\subsubsection{SPARC status has no correlation with liver metastasis burden}

To see whether SPARC influences metastatic spread, we performed metastatic score analysis in H\&E stained liver sections of tumor bearing KC-SPARC ${ }^{-/-}(n=15)$ and KC$\operatorname{SPARC}^{\mathrm{wt}}(\mathrm{n}=10)$ mice. The average number of liver metastasis per mouse was assessed 
by counting the number of liver metastases in H\&E stained liver sections. 5 serial sections were used per mouse. Liver metastases ranging from $100-200 \mu \mathrm{m}$ were considered as micro-metastases, and liver metastases with a size larger than $200 \mu \mathrm{m}$ were considered as macro-metastases. Each macro-metastasis was multiplied by 3 for the final count. Finally, the average number of liver metastasis per mouse was calculated by dividing the total number by 5 . This analysis revealed that both $\mathrm{KC}_{-} \mathrm{SPARC}^{-/-}(\mathrm{n}=15)$ and $\mathrm{KC}-S P A R C^{\mathrm{wt}}(\mathrm{n}=10)$ tumor bearing mice cohort have comparable metastatic score $(p=0.14)$.

A.

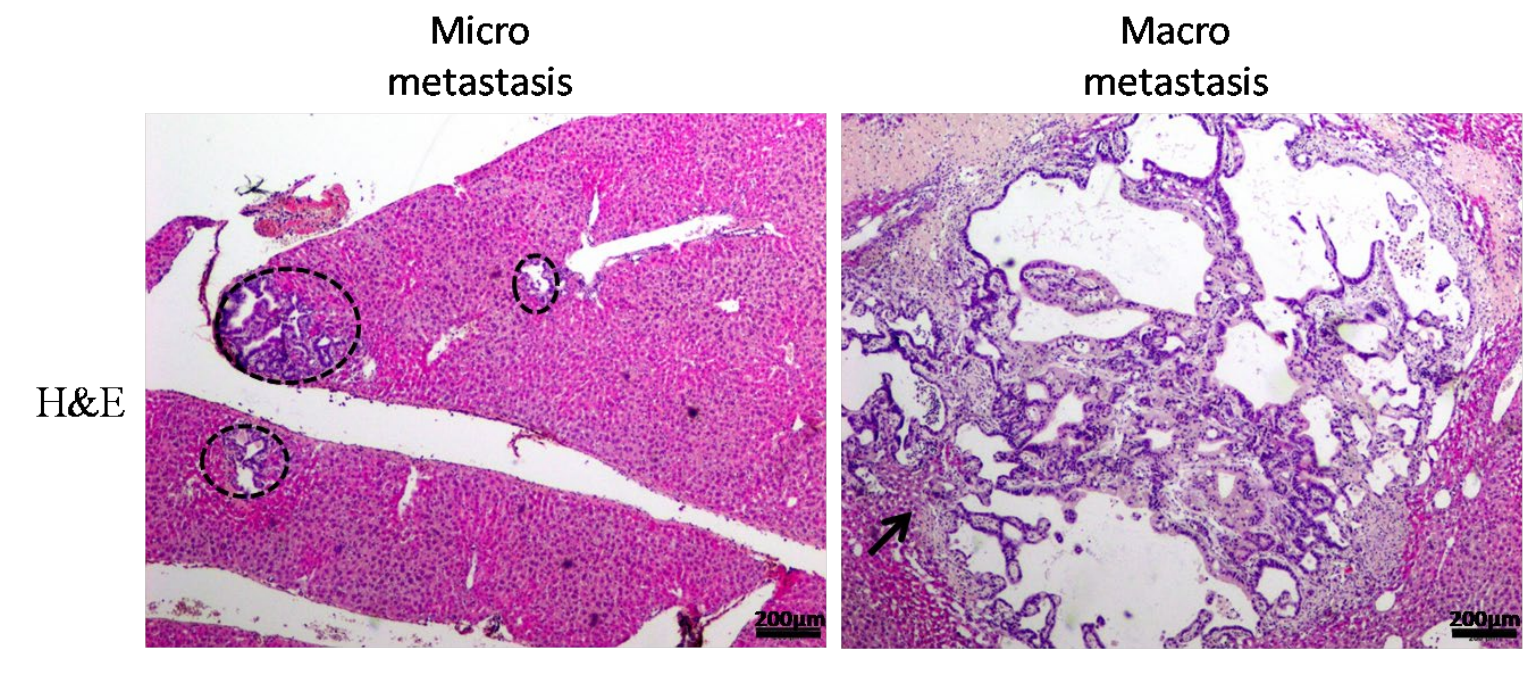

B.

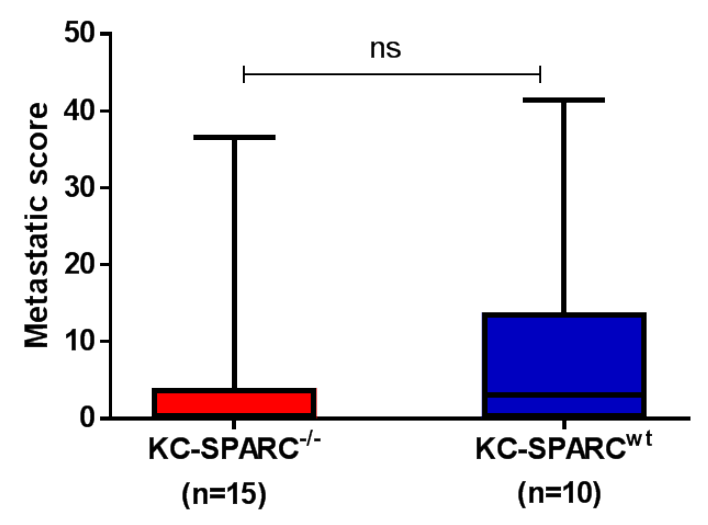

Figure 24: Representative H\&E staining pictures show the micro (left) and macro (right) metastasis in the livers of tumor bearing KC-SPARC ${ }^{\mathrm{wt}}$. (B) Metastatic score comparison of KC-SPARC ${ }^{\mathrm{wt}}$ and $\mathrm{KC}^{-S P A R C}{ }^{-}$ '- mice showing similar metastatic burden for both genotypes $(\mathrm{p}=0.14)$. 


\subsection{Survival analysis}

\subsubsection{Overall survival analysis}

Overall survival analysis of KC-SPARC ${ }^{-/}(\mathrm{n}=90)$ and $\mathrm{KC}^{-S P A R C}{ }^{\mathrm{wt}}(\mathrm{n}=44)$ mice was performed using Kaplan Meier Curve. Overall survival refers to the survival of all mice that died for tumor related health concerns as well as other reasons. The mice were sacrificed using the endpoint criteria such as persistent pain, open wounds, dyspnea, diarrhea, weight loss $>20 \%$, ascites or inactivity. The remaining mice were sacrificed after 20 months. Overall survival analyses revealed that $\mathrm{KC}-\mathrm{SPARC}^{-/}$mice had a shorter survival than KC-SPARC ${ }^{\mathrm{wt}}$ mice (median survival- 390 vs 471 days). However, the difference was not statistically significant ( $p=0.77$; Fig. 25).

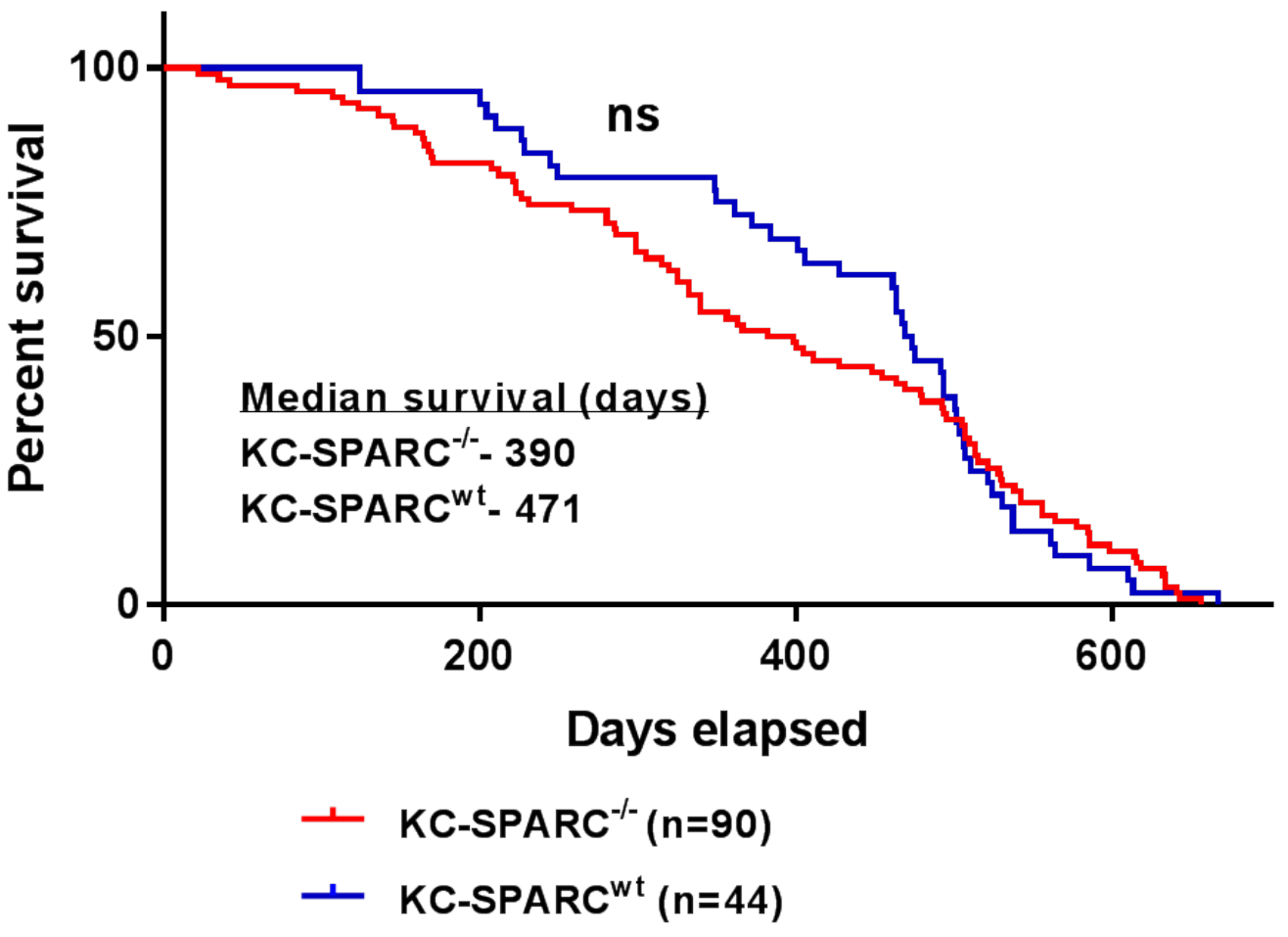

Figure 25: Kaplan Meier curve showing overall survival of KC-SPARC ${ }^{-/-}(\mathrm{n}=90)$ and $\mathrm{KC}^{-S P A R C}{ }^{\mathrm{wt}}(\mathrm{n}=44)$ mice. KC-SPARC ${ }^{\mathrm{wt}}$ and $\mathrm{KC}^{-S P A R C^{-/-}}$have median survival of 471 and 390 days respectively $(\mathrm{p}=0.77)$. Log-rank (Mantel-cox) test was used to assess the statistical significance. 


\subsubsection{Tumor related survival analysis}

Tumor related survival of KC-SPARC ${ }^{-/-}(n=25)$ and KC-SPARC ${ }^{\mathrm{wt}}(\mathrm{n}=16)$ mice was assessed by comparing the survival days of mice which died from tumor-related health concerns. Tumor status was confirmed by visual observation of tumors upon necropsy and subsequent H\&E staining. Tumor related survival analysis shows that tumor bearing $\mathrm{KC}-S P A R C^{-/-}$mice live significantly shorter $(\mathrm{p}=0.02)$. The median survival of tumor bearing $\mathrm{KC}-\mathrm{SPARC}^{-/}$and $\mathrm{KC}-\mathrm{SPARC}{ }^{\mathrm{wt}}$ mice was 280 and 485 days respectively (Fig. 26).

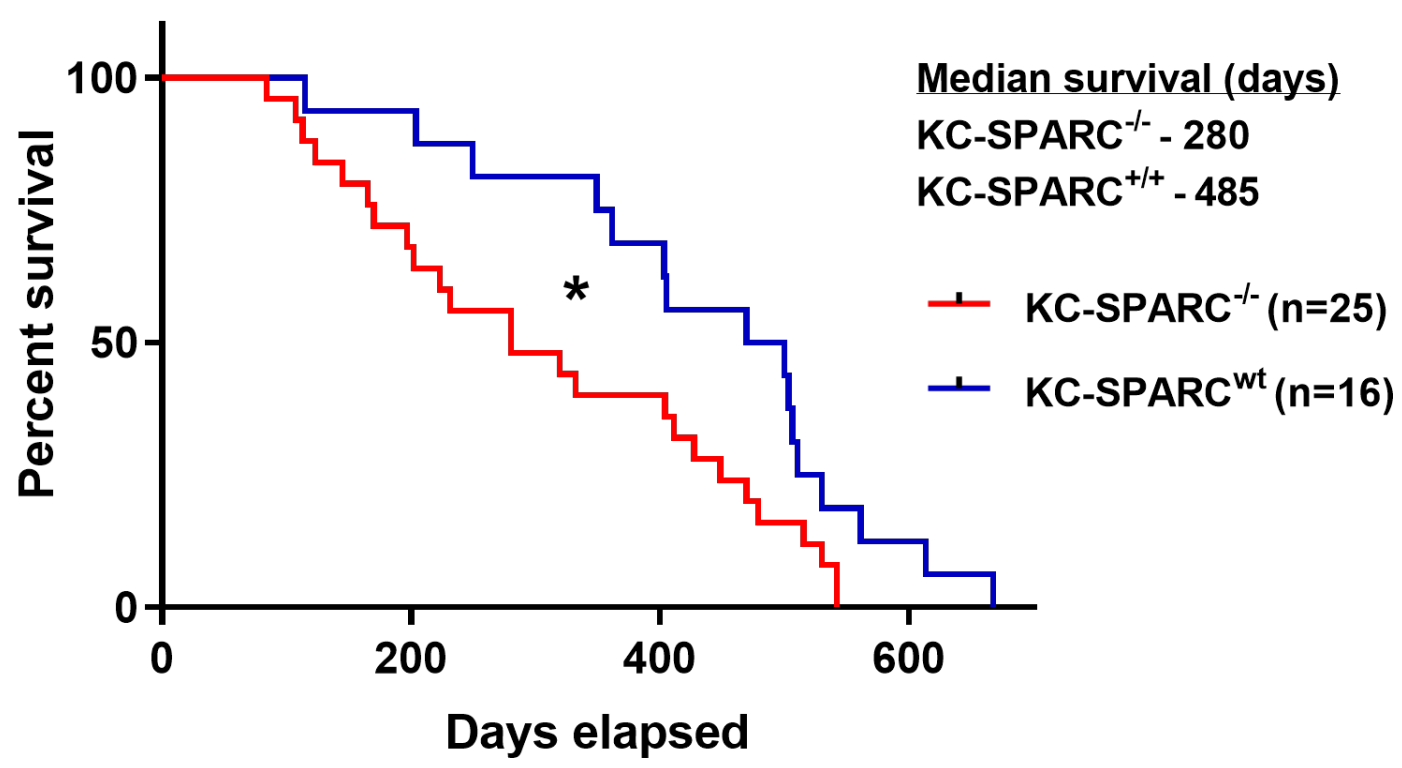

Figure 26: Kaplan Meier Curve of tumor-related survival shows that KC-SPARC ${ }^{\text {wt }}$ have a significantly prolonged survival compared to $\mathrm{KC}^{-S P A R C} \mathrm{C}^{-/}$mice ( 485 days versus 280 days; $\mathrm{p}=0.02$ ). 


\subsubsection{Tumor bearing KC-SPARC ${ }^{-/-}$mice show severe tumor related complications}

Even though $\mathrm{KC}-S P A R C^{-/}$and $\mathrm{KC}-S P A R C^{\mathrm{wt}}$ reveal comparable biological characteristics of their primary tumor and metastatic lesions (proliferation, apoptosis and liver metastasis status), stromal SPARC ablation resulted in a significantly shortened survival. One possible reason for this survival difference might be a more severe disease course and a higher and earlier complication rate in $\mathrm{KC}-S P A R C^{-/-}$mice upon pancreatic tumor development. Table.1 depicts the recorded clinical complications following tumor development that prompted sacrifice of the mice. Even though the single parameters did not reach statistical significance, the accumulation of several factors such as ascites, biliary obstruction and diarrhea occurred more frequently in $\mathrm{KC}^{-S P A R C}{ }^{-/-}$mice and might serve as a feasible explanation for the observed survival difference.

\begin{tabular}{|c|c|c|c|c|c|}
\hline \multirow[b]{3}{*}{ Ascites } & \multicolumn{4}{|c|}{ Tumor } & \multirow[t]{2}{*}{ p-value } \\
\hline & \multicolumn{2}{|c|}{ KC-SPARC $^{-/-}(n=26)$} & \multicolumn{2}{|c|}{ KC-SPARC $^{w t}(n=18)$} & \\
\hline & 4 & $(15 \%)$ & 0 & & $\mathrm{p}=0.13$ \\
\hline Jaundice & 4 & $(15 \%)$ & 1 & $(6 \%)$ & $\mathrm{p}=0.63$ \\
\hline Cachexia & 3 & $(12 \%)$ & 2 & $(11 \%)$ & $\mathrm{p}>0.9$ \\
\hline $\begin{array}{l}\text { Bowel related complaints } \\
\text { (Obstruction, dilation and } \\
\text { ischemic) }\end{array}$ & 3 & $(12 \%)$ & 2 & $(11 \%)$ & $\mathrm{p}>0.9$ \\
\hline $\begin{array}{l}\text { Incidence of } \\
\text { macrometastasis }\end{array}$ & 3 & $(12 \%)$ & 4 & $(22 \%)$ & $\mathrm{p}=0.41$ \\
\hline Abdominal hemorrhage & 2 & $(8 \%)$ & 2 & $(11 \%)$ & $\mathrm{p}>0.9$ \\
\hline Spleen enlargement & 3 & $(12 \%)$ & 1 & $(6 \%)$ & $p=0.63$ \\
\hline Diarrhea & 2 & $(8 \%)$ & 0 & & $\mathrm{p}=0.50$ \\
\hline Hypothermia & 1 & $(4 \%)$ & 1 & $(6 \%)$ & $\mathrm{p}>0.9$ \\
\hline
\end{tabular}

Figure 27: Table shows the comparison of clinical features of tumor bearing $\mathrm{KC}-\mathrm{SPARC}^{-/-}$and KCSPARC $^{\text {wt }}$ at endpoint. 


\subsection{SPARC does not affect gemcitabine delivery and metabolism}

\subsubsection{SPARC mediated collagen deposition does not affect gemcitabine delivery and metabolism in murine pancreatic tumors}

SPARC is a modulator of desmoplasia. In the presented work, I have shown a significant reduction of collagen deposition upon SPARC depletion in murine pancreatic tumors. Extensive ECM deposition (especially collagen) has been shown to impede drug delivery and drug response (Dangi-Garimella et al., 2011; Khan et al., 2016; Olive et al., 2009). Recently, overexpression of collagen genes were found to associated with drug resistance in ovarian cancer (Januchowski et al., 2016; Sherman-Baust et al., 2003).

To investigate whether SPARC mediated ablation of ECM components such as collagen has any effect on delivery and metabolism of gemcitabine, we have treated tumor bearing $\mathrm{KC}-S P A R C^{-/-}(\mathrm{n}=10)$ and KC-SPARC ${ }^{\mathrm{wt}}(\mathrm{n}=6)$ mice with gemcitabine $(100 \mathrm{mg} / \mathrm{kg} /$ body weight) and sacrificed animals $2 \mathrm{~h}$ after the gemcitabine administration. Prior work had shown that peak levels of gemcitabine are detected about $2 \mathrm{~h}$ after intraperitoneal administration (Neesse et al PNAS 2013). Metabolites of gemcitabine were then analyzed in freshly frozen bulk tumor tissue by LC-MS/MS.

Gemcitabine is a prodrug which has to be further metabolized into a functionally active drug by subsequent phosphorylations. Difluorodeoxycytidine ( $\mathrm{dFdC})$ is transported into the cells by human nucleoside transporters (hENTs) and phosphorylated by deoxycytidine kinase ( $\mathrm{dCK}$ ) enzyme to form difluorodeoxycytidine monophosphate (dFdCMP), which is further phosphorylated to form difluorodeoxycytidine diphosphate (dFdCDP) and difluorodeoxycytidine triphosphate (dFdCTP) (Heinemann et al., 1988). $\mathrm{dFdCTP}$ is the cytotoxic form of gemcitabine. Upon incorporation into DNA, it inhibits the function of DNA polymerase and thus terminates chain elongation.

In contrast to previously published data on collagen and drug delivery in murine pancreatic cancer, SPARC mediated collagen deposition in established KC-SPARC ${ }^{\mathrm{wt}}$ tumors did not impede drug delivery or affect metabolism of gemcitabine compared to collagen-poor tumors in $\mathrm{KC}-S P A R C^{-/-}$mice. There was no difference in terms prodrug $(\mathrm{dFdC})$ accumulation $(\mathrm{p}=0.11)$ (Fig. 28B), the amount of activated metabolite (dFdCTP) $(p=0.11)$ (Fig. 28C), and the amount of inactivated metabolite ( $\mathrm{dFdU})(\mathrm{p}=0.79)$ between the collagen-rich $\left(\mathrm{KC}-S P A R C^{\mathrm{wt}}\right)$ and collagen-poor tumors. This finding is surprising and contradicts previous studies which suggested that the extensive ECM (especially 
collagen) deposition could impede drug delivery (Dangi-Garimella et al., 2011; Khan et al., 2016; Olive et al., 2009).

A.
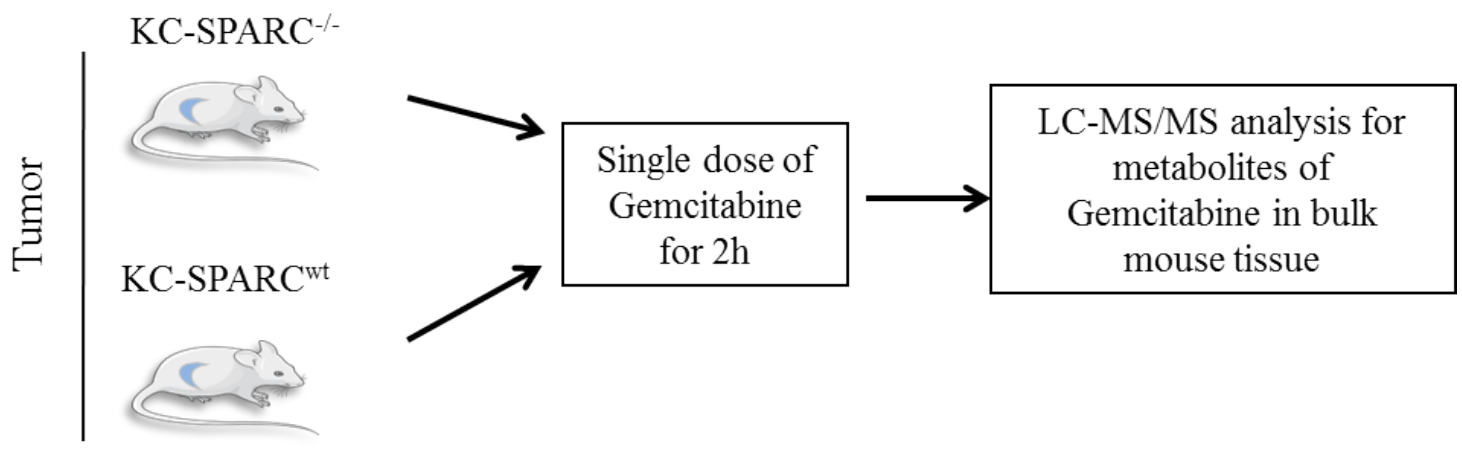

B.

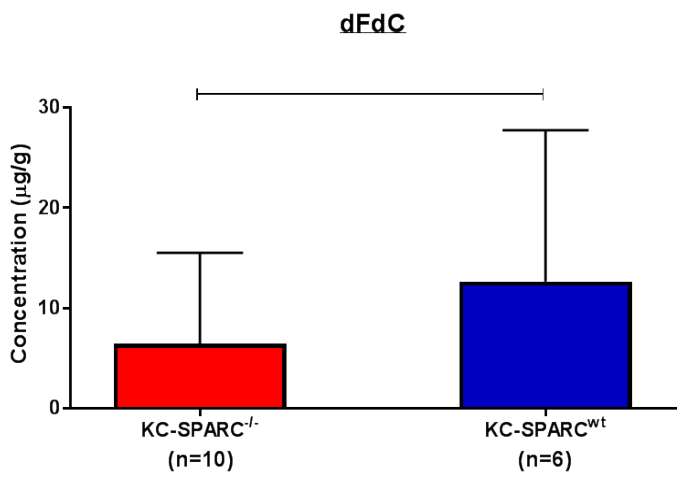

C.

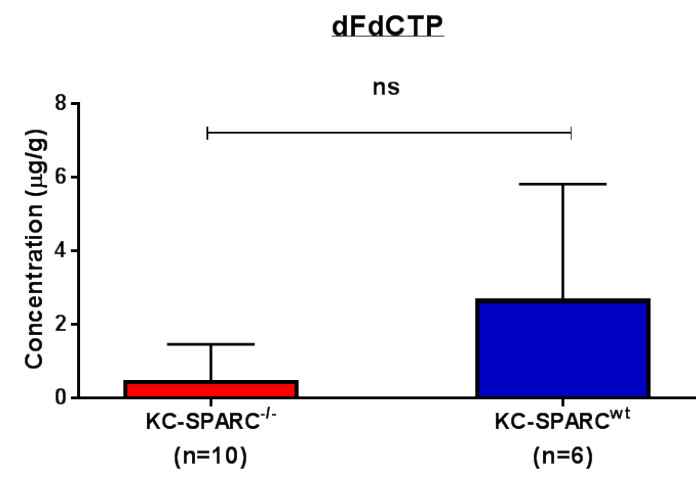

D.

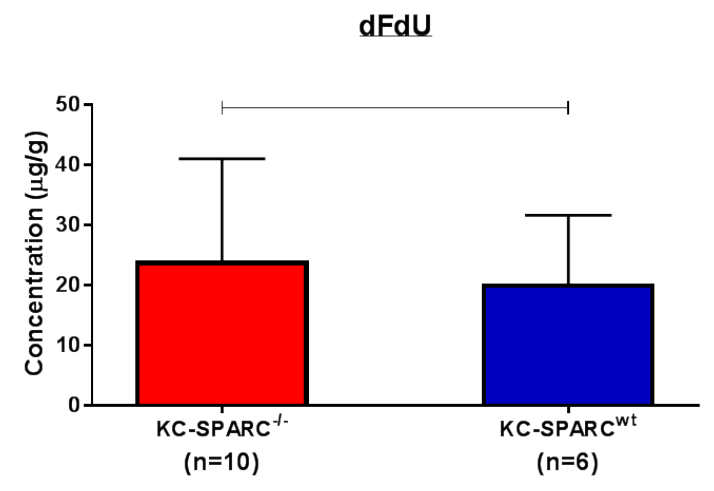

Figure 28: (A) Schematic diagram shows the experimental plan. LC-MS/MS analysis of gemcitabine metabolites (B) dFdC, (C) dFdCTP and (D) dFdU shows that there is no difference in terms of the amount of $\mathrm{dFdC}(\mathrm{p}=0.11), \mathrm{dFdCTP}(\mathrm{p}=0.11)$ and $\mathrm{dFdU}(\mathrm{p}=0.79)$ between collagen-rich tumors (KC-SPARC $\left.{ }^{\mathrm{wt}}\right)$ and collagen-poor tumors $\left(\mathrm{KC}^{-S P A R C}{ }^{-/}\right)$. Mann Whitney test was used to assess the statistical analysis. 


\subsubsection{Overall cellularity but not the SPARC mediated collagen deposition affects drug accumulation and metabolism}

As shown above, SPARC mediated collagen deposition does not affect drug delivery and metabolism of gemcitabine in murine pancreas tumors. Further experiments were aimed to investigate whether drug uptake and metabolism between normal pancreas and different stages of tumorigenesis would differ in order to understand the role of desmoplasia and drug accumulation. In order to achieve this, wildtype mice with healthy pancreata $(n=6)$, mice bearing PanIN lesions $(n=8)$ and tumor bearing KC-SPARC ${ }^{\mathrm{wt}}$ mice $(\mathrm{n}=4)$ were treated with gemcitabine $(100 \mathrm{mg} / \mathrm{kg} /$ body weight $) 2 \mathrm{~h}$ prior to sacrifice, and gemcitabine metabolites were analyzed in freshly frozen bulk tissues by LC-MS/MS.

The analysis revealed a gradual increase in native gemcitabine metabolite ( $\mathrm{dFdC})$ between normal pancreas, PanIN lesions and murine pancreatic tumors derived from $\mathrm{KC}$ SPARC ${ }^{\mathrm{wt}}$ mice (Fig. 29A). However, dFdC was rapidly inactivated to $\mathrm{dFdU}$ in a significant amount in the tumors (Fig. 29C). Hence, there was no significant difference in the amount of activated gemcitabine metabolites ( $\mathrm{dFdCTP}$ ) between normal pancreas, PanIN lesions and tumors (Fig. 29B).

The accumulation of $\mathrm{dFdC}$ was found to be significantly higher in murine tumors $(n=4)$ (15 times more) compared to healthy control pancreas $(n=6)(p=0.009)$. This is surprising due to the fact that the normal pancreas tissue has a much better vascularization than the tumor (Hessmann et al., 2018; Olive et al., 2009). Therefore, one would expect increased accumulation of the pro-drug in normal pancreas compared to hypovascular pancreatic tumors. We hypothesized that the cellularity (both stromal and epithelial cells) might determine LC-MS/MS results for gemcitabine metabolites as an increased number of epithelial and stromal cells might also increase the number of intracellularly measured gemcitabine metabolites. To this end, DAPI staining and IHC for pan-cytokeratin and $\alpha$ SMA were performed to assess the overall epithelial and stromal cellularity. And indeed, an increased cellularity was detected in PanIN lesions and murine tumors compared to normal pancreas. Analysis of DAPI staining revealed that the total number of nuclei was significantly higher in tumor tissue (average 930 nuclei) compared to normal pancreas (average 503 nuclei) (Fig. D \& E) $(p=0.002)$. 
Additionally, IHC-pan-cytokeratin and IHC- $\alpha$-SMA analysis revealed that there is a significant increase in epithelial and mesenchymal cell population in murine tumors compared to PanIN lesions (Fig. $29 \mathrm{~F}-\mathrm{H})(\mathrm{p}=0.04$ and $\mathrm{p}=0.0007$ respectively). Normal pancreas tissue sections were not included in the staining panel as it is well known that both epithelial (ductal) and the fibroblast cell populations are significantly lower in the healthy pancreata.

A.

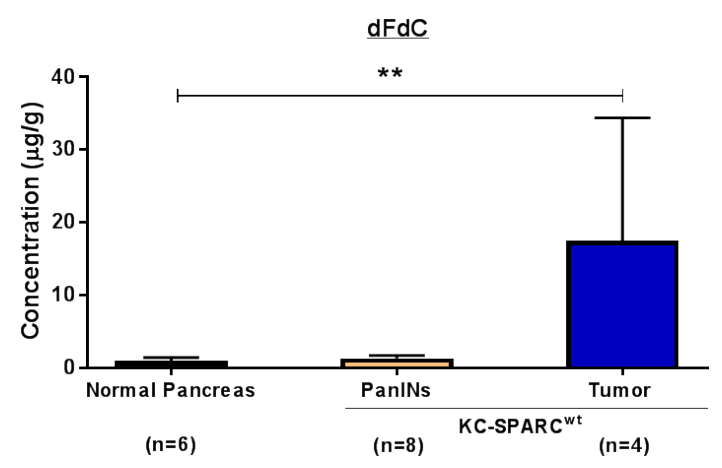

C.

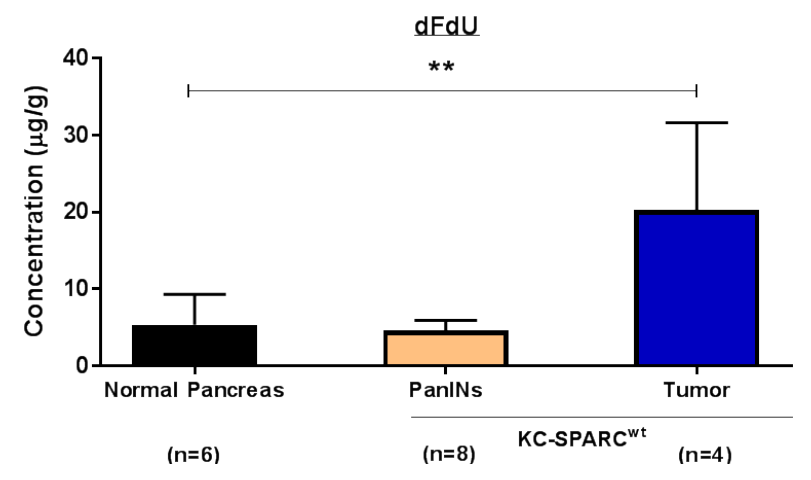

Figure 29: LC-MS/MS analysis of gemcitabine metabolites (A) dFdC, (B) dFdCTP and (C) dFdU in normal pancreas $(n=6)$, PanIN lesions $(n=8)$ and pancreatic tumors $(n=4)$ of $K_{C-S P A R C}{ }^{w t}$ mice. 
D.

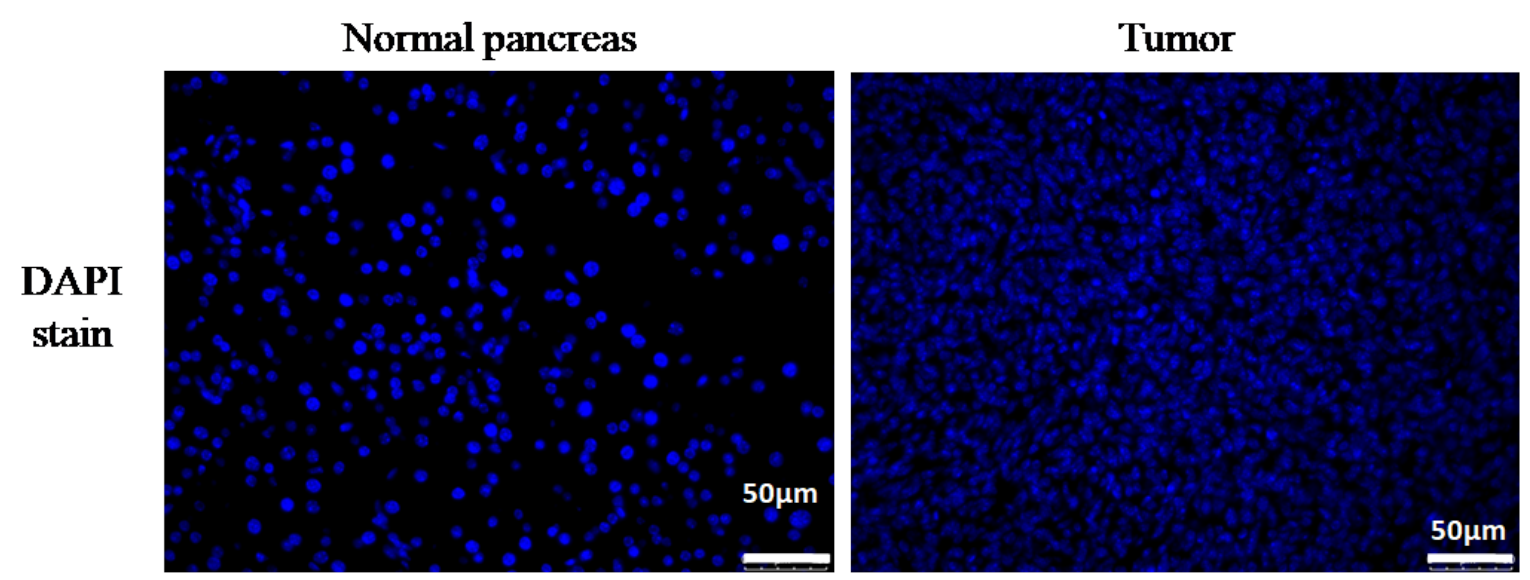

E.

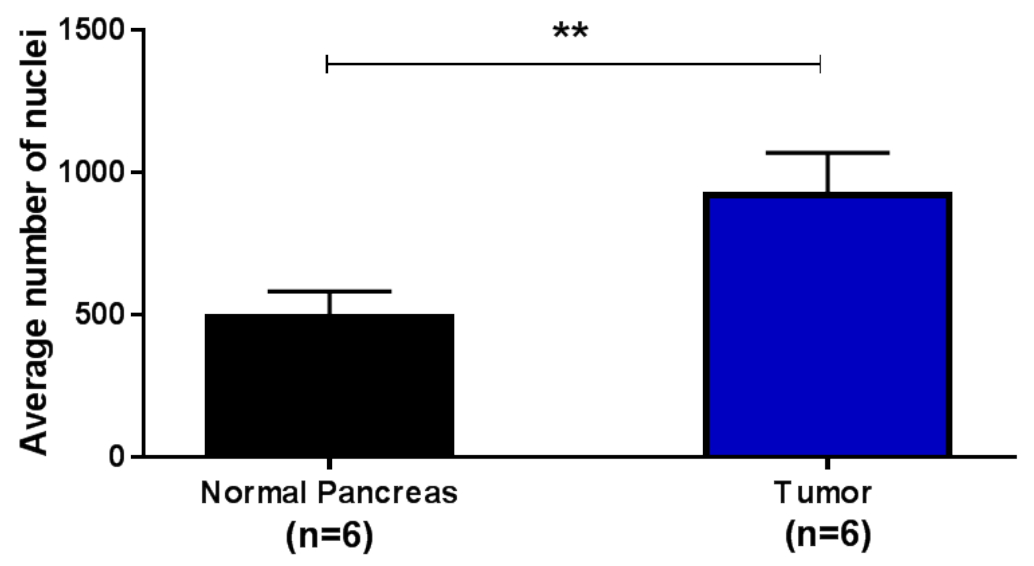

(D) Representative pictures of DAPI staining in control pancreas and KC-SPARC ${ }^{\text {wt }}$ tumor tissue. (E) The quantification of total nuclei in normal pancreas and tumor of KC-SPARC ${ }^{\mathrm{wt}}$ mice shows a significant increase in overall cellularity in tumors compared to normal pancreas $(\mathrm{p}=0.002)$. 
F.

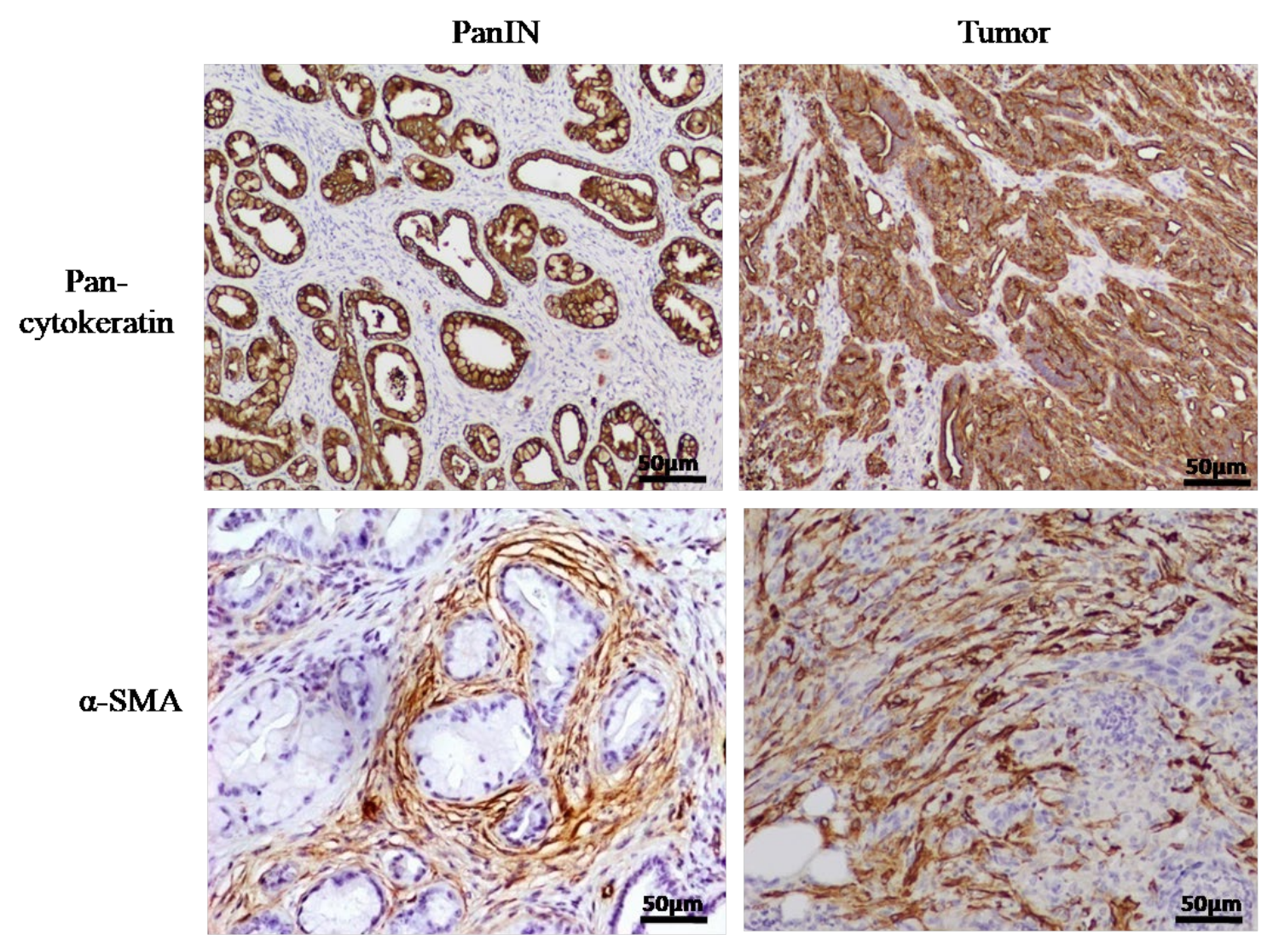

G.

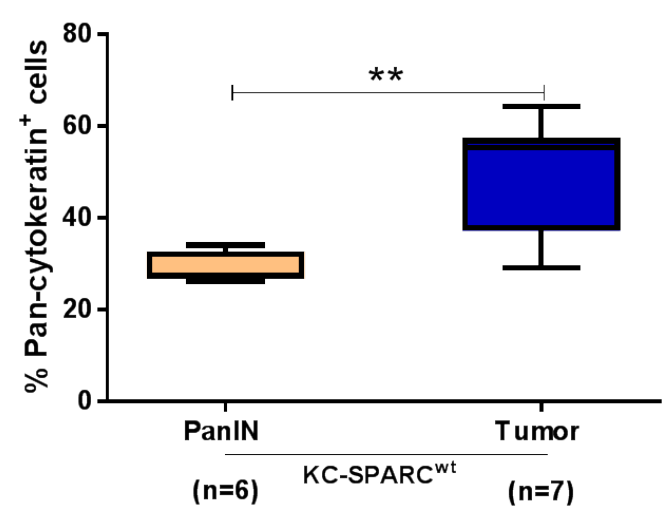

H.

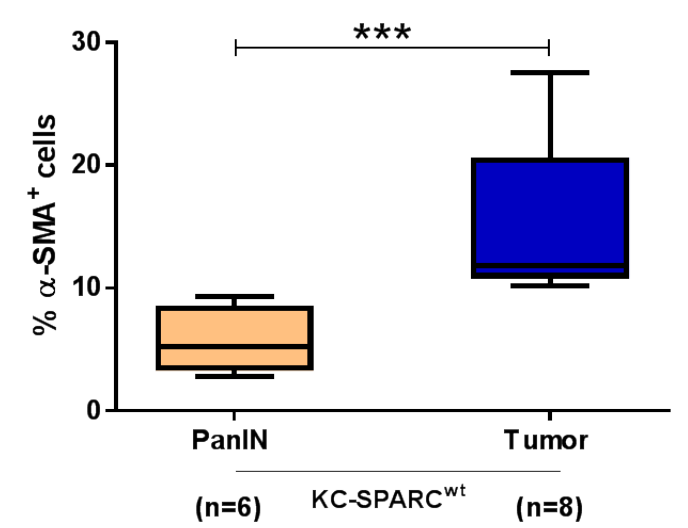

(F) Representative pictures of IHC-pan-cytokeratin and IHC- $\alpha$-SMA in PanIN lesions and tumor tissue shows the increase in cellularity of epithelial and stromal compartment between PanIN lesions and tumors. (G\&H) Quantification of pan-cytokeratin and $\alpha$-SMA showing the increase in cellularity of epithelial and stromal compartments in PanIN lesions compared to tumors. Mann Whitney test was used to assess the statistical significance. $* * \mathrm{p} \leq 0.01$ and $* * * \mathrm{p} \leq 0.005$. 


\section{Results}

\subsubsection{Gemcitabine is enzymatically inactivated by drug metabolizing enzymes}

Overexpression of the inactivating enzymes of gemcitabine is directly associated with drug resistance. Cytidine deaminase (CDA), and Cytosolic 5-Nucleotidase 1A (Nt5c1A) are the two major inactivating enzymes of gemcitabine nucleosides (Mini et al., 2006). In the previous part I had shown that a significant amount of pro-drug accumulated in tumor tissue compared to the normal and pre-neoplastic tissues. Although there was a trend towards more dFdCTP in tumor tissues, no significant difference of $\mathrm{dFdCTP}$ between the normal pancreas tissue, PanIN lesions and murine tumors was detected by LC-MS/MS (Fig. 29B). One possible explanation for this could be that rapid inactivation of $\mathrm{dFdC}$ occurred through CDA ( $\mathrm{dFdC}$ to $\mathrm{dFdU}$ ) and Nt5c1A (dFdCMP to $\mathrm{dFdC}$ ). Indeed, IHC confirmed the strongest expression of $\mathrm{Nt} 5 \mathrm{c} 1 \mathrm{~A}$ and $\mathrm{CDA}$ in murine tumors compared to PanINs and normal pancreas tissue (Fig. 30).
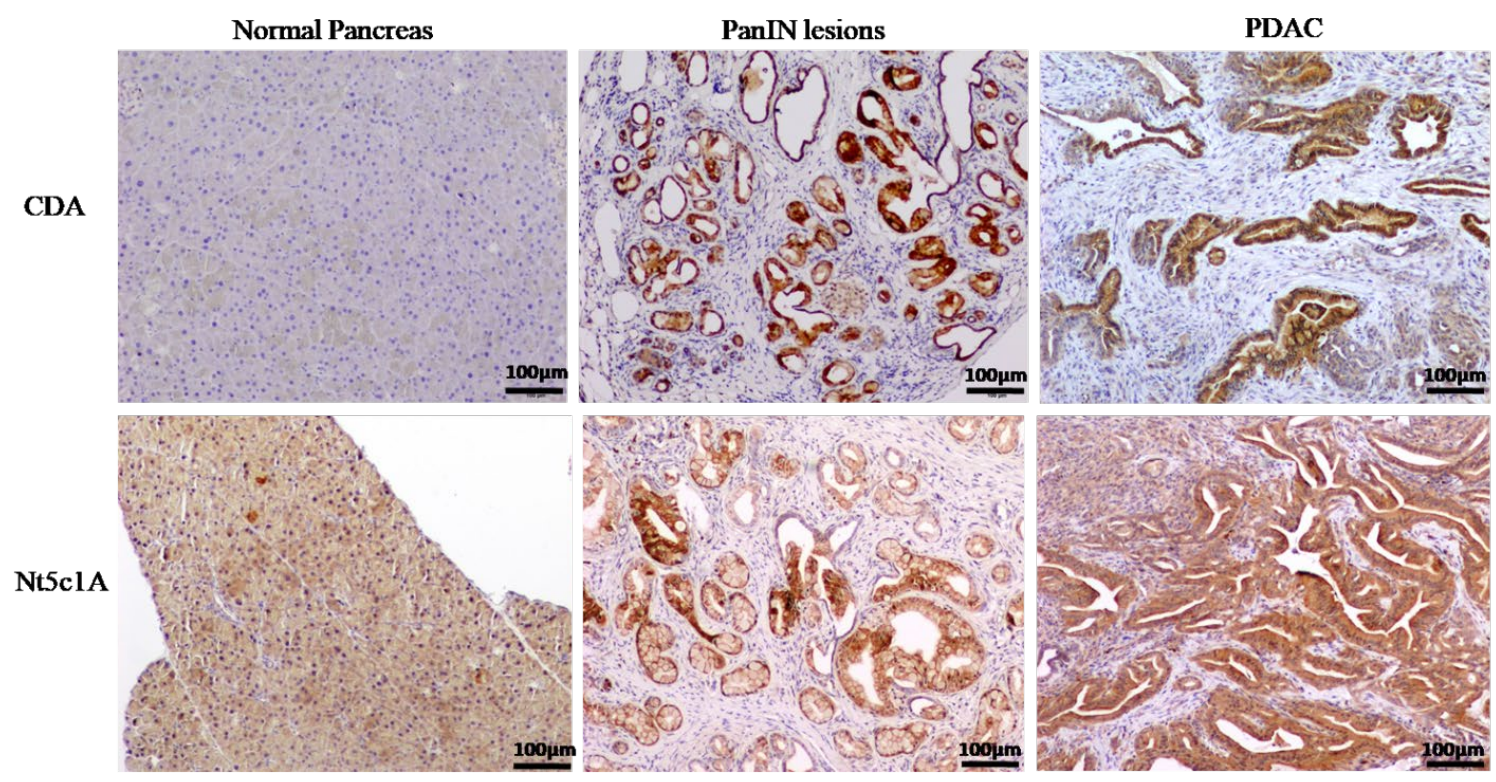

Figure 30: Representative pictures of IHC-CDA and IHC-Nt5c1A in normal pancreas, PanIN lesions and tumors show increased expression in PanIN lesions, and robust overexpression in murine tumor compared to normal pancreas tissue. 


\section{Discussion}

The tumor microenvironment (TME) in PDAC is histologically composed of various components such as acellular and cellular stromal compartments. Each compartment has been shown to contribute to the initiation and progression of the disease. However, depletion of certain cellular components such as CAFs in several GEMMs of PDAC has led to a more aggressive and undifferentiated phenotype suggesting that certain components of the TME are tumor-suppressive whereas others are tumor-promoting (Özdemir et al., 2014; Rhim et al., 2014). SPARC is a matricellular protein that has been shown to be involved in various cellular processes such as development and wound healing. Aberrant SPARC expression is associated with various diseases such as renal fibrosis and arthritis. SPARC expression has also been described in various tumors. In PDAC, SPARC is overexpressed by activated fibroblasts of the tumor stroma and correlates with poor clinical outcome (Infante et al., 2007; Mantoni et al., 2008). Though, extensive research has been done on SPARC, its exact role in PDAC and the mechanism involved are yet a matter of debate. Therefore, analyzing the role of SPARC during different stages of PDAC tumorigenesis using GEMMs may shed light into the role of SPARC in PDAC.

\subsection{Advantages and disadvantages of genetically engineered mice used in this study}

In our study, we have provided a detailed insight into the phenotypic characterization of the mouse models including SPARC ${ }^{-/-}, \mathrm{KC}-S P A R C^{-/-}$and $\mathrm{KC}^{-S P A R C}{ }^{\mathrm{wt}}$. As described previously (Gilmour et al., 1998; Norose et al., 1998b; Yan and Sage, 1999), we have observed SPARC knockout related phenotypes such as fragile bones and cataractogenesis in both SPARC ${ }^{-/-}$and $\mathrm{KC}^{-S P A R C} \mathrm{~S}^{-/-}$mice. In addition, we have observed a trend towards reduced overall body weight as well as organ weight in SPARC knockout mice compared to SPARC ${ }^{\mathrm{wt}}$. Though these phenotypes are not lethal, SPARC $\mathrm{S}^{-/}$mice may be more susceptible to tumor related complications such as weight loss, cachexia and ascites. This limitation could be circumvented by using a pancreas specific SPARC knockout mouse instead of a germline SPARC knockout system. Thus, collagen impairment in the muscle, bone and other tissues such as the cornea would not be affected. However, major advantages of the $\mathrm{KC}$ mouse model are the slow progression of ADM and PanIN lesions that eventually progress to frank carcinomas only after $>8$ 10 months. In addition, pancreatic precursor lesions are surrounded by a dense 
extracellular matrix that resembles the histological features in human preneoplasia. Thus, the $\mathrm{KC}$ mouse models allow a detailed histological and molecular characterization at different stages of tumorigenesis, and eventually tumor growth and metastasis upon SPARC depletion. This prompted us to allocate KC mice into different age groups with well characterized preneoplastic lesions. However, a major drawback of p48-Cre driven mouse models is the fact that oncogenic Kras mutations are initiated during embryonic stages at day 8.5, and all p48 expressing cells bear an activating Kras ${ }^{\mathrm{G} 12 \mathrm{D}}$ mutation. This is in stark contrast to human PDAC in which the tumor normally develops only at one site in the pancreas (head, body or tail) and from one or very few cell clones.

From a technical and experimental point of view, GEMMs are expensive and time consuming due to multiple crosses and genotyping that is required to obtain the correct genotypes.

\subsection{Cancer associated fibroblast in PDAC}

The significance of CAFs and their ability to modulate PDAC stroma by secreting extensive amount of ECM proteins, MMPs and tissue inhibitors of MMPs have been unnoticed for decades (Phillips et al., 2003). Later, the contribution of CAFs in PDAC progression and the potential cross talks between neoplastic cells and CAFs have been described by many in vitro and in vivo studies. In vitro co-culture experiments have shown that both CAFs and tumor cells reciprocally contribute to their proliferation and migration which indeed results in tumor progression and metastasis (Kikuta et al., 2010; Vonlaufen et al., 2008b). Studies have also explored the signaling pathways involved in the interaction between these two different compartments. Tumor cells are known to influence the CAFs to produce more ECM through TGFß1, FGF2, and the increased proliferation of CAFs is known to be associated with the secretion of tumor PDGF, respectively (Bachem et al., 2005; Yoshida et al., 2004; Yoshida et al., 2005). The crosstalk between CAFs and tumor cell was further validated by in vivo studies using xenograft models in which an increase in tumor growth was reported upon co-injection of CAFs and tumor cells compared to tumors cells alone. It has been also shown that the amount the tumor cells was also significantly increased upon co-injection which is supporting the fact that CAFs contribute to the proliferation of tumor cells (Bachem et al., 2005). Similar results were obtained in an orthotopic model in which co-implantation of human PDAC cells and human PSCs led to significant increase in tumor growth and metastasis (Hwang et al., 2008). Considering the tumor promoting roles of CAFs in PDAC, many groups 
have recently attempted to target CAFs to improve therapeutic outcome. Even though few initial studies in preclinical models have shown convincing results (Olive et al 2009), most of those studies failed as the depletion of CAFs resulted in unfavorable tumor characteristics. This might be due to the fact that CAFs also have certain anti-tumorigenic roles (Froeling et al., 2011; Özdemir et al., 2014; Rhim et al., 2014).

Initially, CAFs were considered a homogenous cell population in tumor biology. But later researchers have realized that $\mathrm{CAF}$ population express a wide range of molecular markers and were also thought to be functionally divergent. Further studies have explored the subtypes of CAFs based on different parameters including the expression profile, origin (locally recruited versus bone marrow derived), and their subsequent roles in PDAC (Öhlund et al., 2014; Sugimoto et al., 2006). More recently, Öhlund and colleagues have subclassified CAFs into myofibroblasts and inflammatory fibroblasts based on the expression of different markers and their location of distribution. The group distinguished myofibroblasts from inflammatory fibroblasts based on high expression of $\alpha$-SMA and their proximity with the neoplastic cells (myoCAFs), while the inflammatory fibroblasts (iCAFs) were shown to express mostly inflammatory cytokines such as IL-6 and were located distally from the neoplastic lesions (Öhlund et al., 2017).

We hypothesized that SPARC might indeed determine specific functions in CAFs, as SPARC is predominantly expressed by CAFs during PDAC tumorigenesis. Similar to protumorigenic properties of CAFs, SPARC has been shown to enhance the invasiveness of the tumor cells (Guweidhi et al., 2005). Considering the fact that SPARC is a potent modulator of interaction between tumor and stromal cells, we hypothesized that SPARC may determine the fibroblast's pro or anti-tumorigenic nature. Consequently, we anticipated a direct effect on CAF population upon SPARC depletion in our mouse model.

To differentiate between cell-autonomous and non-cell autonomous effects of SPARC, we first investigated the role of SPARC in epithelial cells. Wound healing assays performed on primary PanIN cells revealed that endogenous SPARC expression has no direct effect on phenotypic properties of pre-neoplastic epithelial cells. This was not surprising due to the fact that there is hardly any expression of SPARC in PanIN cells itself. Similar results were obtained in wound healing assay performed on fibroblasts isolated from $\mathrm{KC}-\mathrm{SPARC} \mathrm{wt}^{\mathrm{wt}}$ and $\mathrm{KC}-\mathrm{SPARC}^{-/-}$mice. Therefore, it appears that SPARC 
had no autocrine effects on both epithelial as well as stromal cells. To investigate whether CAF derived SPARC has paracrine effects on surrounding epithelial cells, co-culture assays with supernatant from CAFs on epithelial cells were performed. To this end, PanIN cells from $\mathrm{KC}^{-S P A R C} \mathrm{~S}^{-/}$mice were incubated with supernatant from both $\mathrm{KC}$ SPARC $^{\mathrm{wt}}$ and $\mathrm{KC}^{-S P A R C}{ }^{-/-}$fibroblasts. Both $\mathrm{KC}^{-S P A R C}{ }^{\mathrm{wt}}$ and $\mathrm{KC}-S P A R C^{-/-}$fibroblast conditional media showed no measurable phenotypic effect on epithelial cells. However, a potential limitation of our in vitro experiments might be the 2D design. CAFs have been shown to exhibit different expression profile upon mono and co-culturing with tumor cells. In addition, CAFs may also require tumor cells in the proximity in order to achieve their original expression profile as seen in vivo. Thus, the stimulation with conditional medium may not fully recapitulate the in vivo. 3D organoid co-cultures would be the appropriate model to study this effect.

Still, our in vitro results are in line with our in vivo findings from $\mathrm{KC}$ mice that SPARC has no effect on PanIN and tumor progression. In our model, the disease initiation and progression led to a gradual increase of the number of CAFs regardless of the SPARC expression. Although we did not observe a direct effect on the fibroblast cell population, loss of SPARC significantly changed the ECM composition by reducing the amount of collagen within preneoplastic lesions and murine tumors. Surprisingly, these alterations in ECM composition did not show significant effects on tumor biology.

\subsection{SPARC mediated ECM deposition and PDAC progression}

The ECM in PDAC is a highly complex network composed of various acellular constituents such as collagen, laminin, fibronectin and proteoglycons which has been shown to regulate various vital cellular functions (Theocharis et al., 2016). SPARC has been shown to modulate the interaction between cellular and acellular (ECM) compartments (Bornstein, 1995; Brekken and Sage, 2000).

In the existing literature, various partly opposing roles of SPARC have been described in different tumor entities. There are certain factors which determine the functions of SPARC in different systems such as the cellular origin of the protein, the amount of secretion and the availability of domains for receptor binding. To this end, SPARC interacts with soluble (i.e growth factors) and insoluble components (collagen, fibronectin etc.,) and is involved in a wide range of cellular functions. In 2003, Brekken et al., have performed a study in which pancreatic tumor cells were implanted 
subcutaneously in $\mathrm{SPARC}^{-/-}$and SPARC ${ }^{\mathrm{wt}}$ mice. The authors reported that tumor growth, volume and weight was increased in $\mathrm{SPARC}^{-/-}$compared to SPARC ${ }^{\mathrm{wt}}$ mice indicating a tumor-suppressive role of SPARC (Brekken et al., 2003). Using a GEMM of PDAC, we first explored the expression pattern of SPARC in the KC model. In the KC mouse model, SPARC was mainly expressed by CAFs that either surrounded PanINs or tumor cells, while there was hardly any expression in epithelial (PanIN or tumor cells) compartment as such. An almost identical expression profile was described in human PDAC where SPARC was found to be expressed by peritumoral fibroblasts (Infante et al., 2007; Mantoni et al., 2008; Sinn et al., 2014). Therefore, we believe that GEMMs of PDAC, in this case $\mathrm{Kras}^{\mathrm{G} 12 \mathrm{D}}$ driven model is more appropriate to recapitulate the human situation, especially in terms of the tumor microenvironment. Interestingly, SPARC has been characterized as one of the markers of activated stroma and its overexpression by stromal fibroblast is correlated with poor clinical outcome in the patients (Infante et al., 2007; Mantoni et al., 2008; Moffitt et al., 2015). However, overexpression of SPARC in activated fibroblasts in the $\mathrm{KC}$ model has not resulted in enhanced tumor progression. Our data is in line with previously published observations in the KPC model, in which SPARC ablation resulted in a similar frequency of liver metastases (Neesse et al., 2014). Comparing our data with the findings of Moffitt et al., we hypothesize that SPARC alone may not contribute to an activated tumor stroma. Rather, the combination of different markers such as CCL13, CCL18, Gelatinase B, Stromelysin 3 and SPARC resulted in the "activated" stroma subtype that was associated with significantly shortened survival in patients. Therefore, clinical and histopathological data that suggest that SPARC is associated with a poor prognosis in PDAC patients might not necessarily reflect protumorigenic functions of SPARC itself but rather SPARC as a marker for desmoplasia.

Mechanistically, SPARC is involved in assembly, folding and secretion of collagen (Martinek et al., 2006). In $\mathrm{KC}-S P A R C^{-/-}$mice, collagen deposition was significantly reduced upon SPARC depletion. Different types of collagen have been shown to confer to tumor growth and progression (Aguilera et al., 2014). However, in our model significant reduction in collagen deposition in $\mathrm{KC}-\mathrm{SPARC}^{-/-}$mice did not affect both disease initiation and progression. Based on our data, one could hypothesize that collagen serves as a passive bystander during carcinogenesis, at least in the $\mathrm{KC}$ mouse model that was used in this study. Alternatively, as collagen deposition was not completely absent in $\mathrm{KC}-\mathrm{SPARC}^{-/-}$mice, there might be a critical amount of collagen that drives PanIN and 
tumor progression that was still present despite the ablation of SPARC. In addition, HA deposition was not affected by SPARC expression. HA is an important stromal constituent of the ECM in PDAC which was shown to promote disease progression and to contribute to drug resistance (Provenzano et al., 2012). Although we did not observe differences in terms of overall survival between $\mathrm{KC}^{-S P A R C} \mathrm{wt}^{\mathrm{wt}}$ and $\mathrm{KC}^{-S P A R C}{ }^{-/-}$mice, tumor bearing $\mathrm{KC}^{-S P A R C^{-/-}}$lived significantly shorter than tumor bearing $\mathrm{KC}-\mathrm{SPARC}{ }^{\mathrm{wt}}$ mice. However, the detailed preclinical analysis did not show any meaningful differences in terms of proliferation, apoptosis, angiogenesis and frequency of liver metastasis. Still, it became apparent that ${\mathrm{KC}-S P A R C^{-/-}}$mice showed more severe tumor associated symptoms such as jaundice, ascites and diarrhea. Therefore, we hypothesize that germline SPARC knockout mice tolerate pancreatic tumor burden less due to intrinsic abnormalities such as metabolic disturbances, ascites development and more severe jaundice.

Intriguingly, the association between SPARC and ascites formation has been previously described in experimental ovarian cancer. Here, host specific SPARC plays a tumor suppressing role by normalizing the ovarian tumor stroma through decreasing the protumorigenic and pro-metastatic factors in ascites (Said et al., 2007). Said and colleagues have explored the mechanism by which SPARC modulates the biochemical composition of the ascitic fluid using syngeneic mouse models. In this study, the authors injected either control ID8 cells or ID8 cells overexpressing VEGF in SPARC ${ }^{-/-}$and SPARC $\mathrm{SP}^{+/+}$ mice. They observed an increased tumor growth, increased incidence of ascites, increased ascites fluid volume and decreased survival in $\mathrm{SPARC}^{-/-}$mice which received ID8+VEGF cells. Furthermore, this study revealed that SPARC reduces the proliferation and invasion of ID8 cells by downregulating the VEGF-MMP levels. Additionally, the ascitic fluid of SPARC ${ }^{-/-}$mice was reported to have higher amounts of pro-tumorigenic inflammatory factors (Said et al., 2007). The positive feedback loop between MMPs and VEGF has been previously reported (Belotti et al., 2003). Thus quantification of VEGF level using ELISA or mRNA expression analysis in the ascitic fluid of KC-SPARC ${ }^{\mathrm{wt}}$ and $\mathrm{KC}-\mathrm{SPARC}^{-/-}$mice may provide further insight into mechanisms by which the increased ascites incidence in $\mathrm{KC}-\mathrm{SPARC}^{-/-}$mice had occurred. For this study, ascites was unfortunately not routinely collected for further analysis.

Even though there was no obvious evidence showing the relationship between SPARC and increased incidence of jaundice, there is evidence suggesting a strong correlation 
between the altered collagen level or mutation in collagen genes and jaundice incidence (Mizuguchi et al., 2005; Tomotaki et al., 2016). However, the exact mechanism has not been elucidated so far.

\subsection{SPARC and angiogenesis}

In general, PDAC is a highly desmoplastic and hypovascular tumor. This hypovascularity causes reduced tumor perfusion which is further decreased by physical compression of the existing tumor vessel by the ECM matrix and the high colloidal tumor pressure. These observations have led to the hypothesis that the abundant tumor stroma is one of the main reasons for the failure of chemotherapies (Olive et al., 2009). Therefore, various experimental attempts have been made to deplete the tumor stroma and increase vascular perfusion thus allowing drugs to enter the tumor more easily. SPARC has been extensively studied in the field of vascular biology and has been referred to as the regulator of angiogenesis (Sage et al., 1984, 1989). Previous studies have shown the antiangiogenic role of SPARC in various tumors entities such as ovarian cancer, neuroblastoma and gastric cancer (Chlenski et al., 2010; Said et al., 2007; Zhang et al., 2012). In ovarian cancer, SPARC has been shown to downregulate VEGF and MMPs in order to achieve the anti-angiogenic activity (Said et al., 2007).

SPARC has also been previously shown to interact with VEGF and thus regulate the proliferation and migration of endothelial cells (Kupprion et al., 1998; Raines et al., 1992). Prior to this work, it could be hypothesized that SPARC depletion may result in enhanced angiogenesis and possibly improved perfusion of murine PDAC tumors. Therefore, mean vessel density was assessed by analyzing immunoreactivity of CD31 in tumor tissues of KC-SPARC ${ }^{-/}$and $\mathrm{KC}-S P A R C^{\mathrm{wt}}$ mice. Surprisingly, no changes in the number of tumor vessels were observed upon SPARC depletion. However, the finding that SPARC ablation resulted in a higher frequency of malignant ascites in tumor bearing mice may still indicate an anti-angiogenic role of SPARC that was abolished following SPARC depletion. Further experimental studies such as a detailed biochemical work-up of ascites and serum samples for secreted factors such as VEGF may further help to investigate this hypothesis in mice. From a clinical point of view, it is interesting to note that only a fraction of patients with PDAC develop malignant ascites during the end stages of the tumor disease. It remains to be determined whether these patients have any differences in global or tumor-associated SPARC expression. Again, this could be interrogated by a clinical proof of concept study where serum samples from patients with 
and without ascites, but comparable tumor stages are analyzed for circulating SPARC, VEGF and other growth factors.

\subsection{SPARC mediated desmoplasia and drug delivery}

Previous studies have described the association between the extensive amount of ECM deposition and drug resistance in PDAC. Various components of ECM such as collagen and hyaluronan have been thought to impede drug delivery by acting as a physical barrier (Jacobetz et al., 2013; Minchinton and Tannock, 2006; Provenzano et al., 2012). In addition to the extrinsic resistance, the desmoplastic stroma of PDAC also has been shown to confer to intrinsic resistance by differentially regulating the cellular enzymes involved in gemcitabine metabolism pathway (Dangi-Garimella et al., 2011, 2013; Hessmann et al., 2018).

Several investigators have used various GEMMs to assess different compounds that target the tumor stroma. For example, Cilengitide, an angiogenesis inhibitor, and Verapamil, a $\mathrm{Ca}^{++}$channel blocker, were co-administered and resulted in improved vascular function, intratumoral gemcitabine accumulation and therapeutic response. There are a number of additional stromal targets such as TGF- $\beta$ using the inhibitor LY364947, HA using the enzyme PEGPH20, or collagen maturation using the lysyl oxidase (LOX) inhibitor that have been experimentally probed in various mouse models with success (Jacobetz et al., 2013; Kano et al., 2007; Miller et al., 2015; Provenzano et al., 2012; Thompson et al., 2010). However, none of these findings could be successfully translated in phase III trials in humans, and so far, no approved anti-stromal drug is currently available in the clinical routine for PDAC patients. To this end, PEGPH20 might represent the most promising candidate right now. A phase III study (NCT02715804) has recently been approved, and patients with high intratumoral HA levels are randomized to nab-paclitaxel/gemcitabine \pm PEGPH20. This trial will show whether the biophysical drug delivery hypothesis in PDAC will translate to patient care.

Another explanation for the failure of chemotherapies has recently been discovered in the Neesse laboratory. Experimental data from GEMMs indicate that CAFs, compared to epithelial tumor cells, metabolize and intracellularly store large amounts of gemcitabine metabolites that are not available for tumor cells anymore. This phenomenon was termed "drug scavenging" and may explain why tumor cells are very sensitive 
towards the chemotherapeutic drug gemcitabine in vitro, but fail to undergo apoptosis when surrounded by a dense, fibroblast-rich environment (Hessmann et al., 2018).

In my thesis, I explored whether SPARC or SPARC mediated desmoplasia affects gemcitabine delivery and metabolism by employing LC-MS/MS analysis in normal pancreas tissue, bulk preneoplastic tissues, and tumor tissues from $\mathrm{KC}-\mathrm{SPARC}^{-/-}$and $\mathrm{KC}$ $\mathrm{SPARC}^{\mathrm{wt}}$ mice upon gemcitabine treatment. These mice models provided an excellent platform to investigate whether collagen content really affected intratumoral gemcitabine accumulation in preneoplastic tissues as well as murine pancreatic tumors. Surprisingly, the results showed that there is no noticeable difference in terms of intrapancreatic gemcitabine accumulation between tumors with high and low collagen content. In fact, the amount of native gemcitabine $(\mathrm{dFdC})$ accumulation was comparable between tumors with high and low collagen content (KC-SPARC $\left.{ }^{\mathrm{wt}} \mathrm{vs} \mathrm{KC}-\mathrm{SPARC}^{-/-}\right)$. According to our model, the extensive deposition of collagen in KC-SPARC ${ }^{\text {wt }}$ tumor does not affect the delivery and metabolism of gemcitabine. This is contradicting the previous studies which have described collagen as a physical barrier for gemcitabine delivery (Dangi-Garimella et al., 2013; Diop-Frimpong et al., 2011).

We further investigated if the accumulation of gemcitabine is disease stage dependent in order to explore the contribution of desmoplasia for drug accumulation. Completely contradicting the biophysical drug barrier hypothesis, LC-MS/MS results revealed a gradual increase in gemcitabine accumulation dependent from normal pancreas to PanIN lesions and fully developed tumors. Bases on the stromal scavenging theory, we hypothesized that the increase in drug accumulation is rather due to the increase in overall cellularity. And indeed, performing nuclear density analysis (DAPI) and IHC for pancytokeratin and $\alpha$-SMA a significant increase in both epithelial and mesenchymal cells in tumors compared to normal pancreas tissues was detected. The ratio between native gemcitabine $(\mathrm{dFdC})$, inactive gemcitabine $(\mathrm{dFdU})$ and the activated form $(\mathrm{dFdCTP})$ demonstrated that the increased accumulation of native gemcitabine in tumor tissue was eventually not phosphorylated to the active dFdCTP. Instead, the amount of inactive $\mathrm{dFdU}$ increased in tumor tissues indicating an increased activity of gemcitabine inactivating enzymes in tumor tissues compared to preneoplastic pancreas tissue and normal pancreas. 


\subsection{Conclusions}

We hypothesized that SPARC is an important matricellular protein in PDAC contributing to disease progression, ECM remodeling and possibly drug delivery. Therefore, genetic abrogation of SPARC in Kras-driven mouse models and cell lines may directly or indirectly affect tumorigenesis and progression, as well as the delivery of chemotherapeutic drugs such as gemcitabine.

\subsubsection{SPARC and tumorigenesis}

- SPARC depletion lead to significant reduction of collagen deposition in KC$\mathrm{SPARC}^{-/}$mice regardless of the disease stage

- SPARC depletion did not affect progression of the ADM-PanIN-tumor sequence in GEMMs.

- The tumor incidence and liver metastasis rate were unaffected by genetic abrogation of SPARC in KC mice.

- SPARC depletion did not change hallmark characteristics of murine pancreatic tumors including tumor weight, proliferation, apoptosis and angiogenesis.

- In vitro, SPARC abrogation did not affect morphology and proliferation of both epithelial and fibroblast cell lines derived from preneoplastic lesions and murine pancreas tumors.

- The overall median survival of KC-SPARC $\mathrm{Kt}^{\mathrm{wt}}$ and $\mathrm{KC}-\mathrm{SPARC}^{-/-}$mice was similar and was not affected by SPARC abrogation.

- Loss of SPARC significantly shortened survival of tumor bearing KC-SPARC ${ }^{-/-}$ mice compared to $\mathrm{KC}-\mathrm{SPARC}^{\mathrm{wt}}$ mice and was associated with more severe clinical symptoms such as ascites, diarrhea and jaundice.

\subsubsection{SPARC and drug delivery}

- There was no difference in terms of delivery and metabolism of gemcitabine between collagen-rich and collagen-poor tumors $\left({\mathrm{KC}-S P A R \mathrm{C}^{\mathrm{wt}}}^{\mathrm{vs}} \mathrm{KC}^{\mathrm{K}-S P A R \mathrm{C}^{-}}\right.$ $\left.{ }^{\prime-}\right)$.

- There was a striking increase in the accumulation of native gemcitabine in murine tumors (high collagen) compared to normal pancreas tissue (no collagen) contradicting several reports that the desmoplastic reaction impedes gemcitabine delivery. 


\section{Discussion}

- Interestingly, native gemcitabine was inactivated by enzymes such as CDA and $\mathrm{Nt} 5 \mathrm{c} 1 \mathrm{~A}$ that were overexpressed in murine tumors but not normal pancreas tissue resulting in comparable levels of activated gemcitabine metabolites in normal pancreas and murine tumors. 
6. References

\section{References}

Aguilera, K.Y., Rivera, L.B., Hur, H., Carbon, J.G., Toombs, J.E., Goldstein, C.D., Dellinger, M.T., Castrillon, D.H., and Brekken, R.A. (2014). Collagen signaling enhances tumor progression after anti-VEGF therapy in a murine model of pancreatic ductal adenocarcinoma. Cancer Res. 74, 1032-1044.

Aguirre, A.J., Bardeesy, N., Sinha, M., Lopez, L., Tuveson, D.A., Horner, J., Redston, M.S., and Depinho, R.A. (2003). Activated Kras and Ink4a/Arf deficiency cooperate to produce metastatic pancreatic ductal adenocarcinoma. Genes Dev. 3112-3126.

Ahmed, S., Bradshaw, A.-D., Gera, S., Dewan, M., and Xu, R. (2017). The TGF$\beta /$ Smad4 Signaling Pathway in Pancreatic Carcinogenesis and Its Clinical Significance. J. Clin. Med. 6, 5.

Albrecht Neesse, Christian Bauer, Daniel Öhlund, Matthias Lauth, Malte Buchholz, Patrick Michl6, D.A.T. and T.M.G. (2018). Stromal biology and therapy in pancreatic cancer- Ready for clinical translation? Gut.

Alliel, P.M., Perin, J. -P, Jolles, P., and Bonnet, F.J. (1993). Testican, a multidomain testicular proteoglycan resembling modulators of cell social behaviour. Eur. J. Biochem.

Ancrile, B.B. (2009). Oncogenic Ras-Induced Expression of Cytokines in Cancer. Mol Interv 8, 22-27.

Ancrile, B., Lim, K.H., and Counter, C.M. (2007). Oncogenic Ras-induced secretion of IL6 is required for tumorigenesis. Genes Dev.

Anderson, M.W., Reynolds, S.H., You, M., and Maronpot, R.M. (1992). Role of Protooncogene activation in carcinogenesis. In Environmental Health Perspectives, pp. 13-24.

Apte, M. V., Haber, P.S., Applegate, T.L., Norton, I.D., McCaughan, G.W., Korsten, M.A., Pirola, R.C., and Wilson, J.S. (1998). Periacinar stellate shaped cells in rat pancreas: Identification, isolation, and culture. Gut 43, 128-133. 
Apte, M. V., Park, S., Phillips, P.A., Santucci, N., Goldstein, D., Kumar, R.K., Ramm, G.A., Buchler, M., Friess, H., McCarroll, J.A., et al. (2004). Desmoplastic reaction in pancreatic cancer: Role of pancreatic stellate cells. Pancreas 29, 179-187.

Apte, M. V., Wilson, J.S., Lugea, A., and Pandol, S.J. (2013). A starring role for stellate cells in the pancreatic cancer microenvironment. Gastroenterology 144, 1210-1219.

Arnold, S.A., and Brekken, R.A. (2009). SPARC: A matricellular regulator of tumorigenesis. J. Cell Commun. Signal. 3, 255-273.

Arnold, S.A., Rivera, L.B., Carbon, J.G., Toombs, J.E., Chang, C.L., Bradshaw, A.D., and Brekken, R.A. (2012). Losartan slows pancreatic tumor progression and extends survival of SPARC-null mice by abrogating aberrant TGF $\beta$ activation. PLoS One 7.

Arslan, A.A., Helzlsouer, K.J., Kooperberg, C., Shu, X., Steplowski, E., Bueno-demesquita, H.B., Fuchs, C.S., Myron, D., Jacobs, E.J., Lacroix, A.Z., et al. (2010). Anthropometric Measures, Body Mass Index and Pancreatic Cancer: a Pooled Analysis from the Pancreatic Cancer Cohort Consortium (PanScan). Arch Intern Med 170, 791802.

Bachem, M.G., Schneider, E., Groß, H., Weidenbach, H., Schmid, R.M., Menke, A., Siech, M., Beger, H., Grünert, A., and Adler, G. (1998). Identification, culture, and characterization of pancreatic stellate cells in rats and humans. Gastroenterology 115, 421-432.

Bachem, M.G., Schünemann, M., Ramadani, M., Siech, M., Beger, H., Buck, A., Zhou, S., Schmid-Kotsas, A., and Adler, G. (2005). Pancreatic carcinoma cells induce fibrosis by stimulating proliferation and matrix synthesis of stellate cells. Gastroenterology.

Bapiro, T.E., Richards, F.M., Goldgraben, M.A., Olive, K.P., Madhu, B., Frese, K.K., Cook, N., Jacobetz, M.A., David, D.S., Gri, J.R., et al. (2011). A novel method for quantification of gemcitabine and its metabolites 2',2'-diXuorodeoxyuridine and gemcitabine triphosphate in tumour tissue by LC-MS/MS: comparison with 19F NMR spectroscopy. 1243-1253. 
Bardeesy, N., Cheng, K.H., Berger, J.H., Chu, G.C., Pahler, J., Olson, P., Hezel, A.F., Horner, J., Lauwers, G.Y., Hanahan, D., et al. (2006). Smad4 is dispensable for normal pancreas development yet critical in progression and tumor biology of pancreas cancer. Genes Dev. 20, 3130-3146.

Baril, P., Gangeswaran, R., Mahon, P.C., Caulee, K., Kocher, H.M., Harada, T., Zhu, M., Kalthoff, H., Crnogorac-Jurcevic, T., and Lemoine, N.R. (2007). Periostin promotes invasiveness and resistance of pancreatic cancer cells to hypoxia-induced cell death: Role of the $\beta 4$ integrin and the PI3k pathway. Oncogene 26, 2082-2094.

Barker, T.H., Baneyx, G., Cardó-Vila, M., Workman, G.A., Weaver, M., Menon, P.M., Dedhar, S., Rempel, S.A., Arap, W., Pasqualini, R., et al. (2005). SPARC regulates extracellular matrix organization through its modulation of integrin-linked kinase activity. J. Biol. Chem.

Bassuk, J.A., Pichler, R., Rothmier, J.D., Pippen, J., Gordon, K., Meek, R.L., Bradshaw, A.D., Lombardi, D., Strandjord, T.P., Reed, M., et al. (2000). Induction of TGF- $\beta 1$ by the matricellular protein SPARC in a rat model of glomerulonephritis. Kidney Int.

Belotti, D., Paganoni, P., Manenti, L., Garofalo, A., Marchini, S., Taraboletti, G., and Giavazzi, R. (2003). Matrix metalloproteinases (MMP9 and MMP2) induce the release of vascular endothelial growth factor (VEGF) by ovarian carcinoma cells: Implications for ascites formation. Cancer Res.

Biankin, A. V., Waddell, N., Kassahn, K.S., Gingras, M.C., Muthuswamy, L.B., Johns, A.L., Miller, D.K., Wilson, P.J., Patch, A.M., Wu, J., et al. (2012). Pancreatic cancer genomes reveal aberrations in axon guidance pathway genes. Nature 491, 399-405.

Boeck, S., Ankerst, D.P., and Heinemann, V. (2008). The role of adjuvant chemotherapy for patients with resected pancreatic cancer: Systematic review of randomized controlled trials and meta-analysis. Oncology 72, 314-321.

Bornstein, P. (1995). Diversity of function is inherent in matricellular proteins: An appraisal of thrombospondin 1. J. Cell Biol. 
Bos, J.L., Fearon, E.R., Hamilton, S.R., Verlaan-de Vries, M., van Boom, J.H., van der Eb, A.J., and Vogelstein, B. (1987). Prevalence of ras gene mutations in human colorectal cancers. Nature 327, 293-297.

Boyle, P., Hsieh, C.C., Maisonneuve, P., La Vecchia, C., Macfarlane, G.J., Walker, A.M., and Trichopoulos, D. (1989). Epidemiology of pancreas cancer (1988). Int. J. Pancreatol. 5, 327-346.

Bradshaw, A.D. (2009). The role of SPARC in extracellular matrix assembly. J. Cell Commun. Signal. 3, 239-246.

Bradshaw, A.D. (2012). Diverse Biological Functions of the SPARC Family of Proteins. $44,480-488$.

Bradshaw, A.D., Puolakkainen, P., Dasgupta, J., Davidson, J.M., Wight, T.N., and Sage, E.H. (2003). SPARC-null mice display abnormalities in the dermis characterized by decreased collagen fibril diameter and reduced tensile strength. J. Invest. Dermatol.

Bramhall, S.R., Rosemurgy, a, Brown, P.D., Bowry, C., and Buckels, J. a (2001). Marimastat as first-line therapy for patients with unresectable pancreatic cancer: a randomized trial. J. Clin. Oncol.

Bramhall, S.R., Schulz, J., Nemunaitis, J., Brown, P.D., Baillet, M., and Buckels, J.A.C. (2002). A double-blind placebo-controlled, randomised study comparing gemcitabine and marimastat with gemcitabine and placebo as first line therapy in patients with advanced pancreatic cancer. Br. J. Cancer.

Brekken, R.A., and Sage, E.H. (2000). SPARC, a matricellular protein: at the crossroads of cell-matrix communication. Matrix Biol. 19, 569-580.

Brekken, R.A., Puolakkainen, P., Graves, D.C., Workman, G., Lubkin, S.R., and Sage, E.H. (2003). Enhanced growth of tumors in SPARC null mice is associated with changes in the ECM. J. Clin. Invest. 111, 487-495. 
Burris, H.A., Moore, M.J., Andersen, J., Green, M.R., Rothenberg, M.L., Modiano, M.R., Cripps, M.C., Portenoy, R.K., Storniolo, A.M., Tarassoff, P., et al. (1997). Improvements in survival and clinical benefit with gemcitabine as first-line therapy for patients with advanced pancreas cancer: a randomized trial. J. Clin. Oncol. 15, 24032413.

Campbell, S.L., Khosravi-Far, R., Rossman, K.L., Clark, G.J., and Der, C.J. (1998). Increasing complexity of Ras signaling. Oncogene 17, 1395-1413.

Carafoli, F., Bihan, D., Stathopoulos, S., Konitsiotis, A.D., Kvansakul, M., Farndale, R.W., Leitinger, B., and Hohenester, E. (2009). Crystallographic Insight into Collagen Recognition by Discoidin Domain Receptor 2. Structure.

Carpelan-Holmstrom, M. (2005). Does anyone survive pancreatic ductal adenocarcinoma? A nationwide study re-evaluating the data of the Finnish Cancer Registry. Gut 54, 385-387.

Carpeño, J.D.C., and Belda-iniesta, C. (2013). KRAS mutant NSCLC , a new opportunity for the synthetic lethality therapeutic approach. Transl. Lung Cancer Res. 2, 142-151.

Casper, E.S., Green, M.R., Kelsen, D.P., Heelan, R.T., Brown, T.D., Flombaum, C.D., Trochanowski, B., and Tarassoff, P.G. (1994). Phase II trial of gemcitabine (2,2'difluorodeoxycytidine) in patients with adenocarcinoma of the pancreas. Invest New Drugs 12, 29-34.

Ceyhan, G.O., Demir, I.E., Altintas, B., Rauch, U., Thiel, G., Müller, M.W., Giese, N.A., Friess, H., and Schäfer, K.H. (2008). Neural invasion in pancreatic cancer: A mutual tropism between neurons and cancer cells. Biochem. Biophys. Res. Commun.

Chambers, A.F., and Matrisian, L.M. (1997). Changing views of the role of matrix metalloproteinases in metastasis. J. Natl. Cancer Inst.

Chanmee, T., Ontong, P., and Itano, N. (2016). Hyaluronan: A modulator of the tumor microenvironment. Cancer Lett. 
Charbonnier, F., Périn, J.P., Mattei, M.G., Camuzat, A., Bonnet, F., Gressin, L., and Alliel, P.M. (1998). Genomic organization of the human SPOCK gene and its chromosomal localization to $5 \mathrm{q} 31$. Genomics.

Chauhan, V.P., Martin, J.D., Liu, H., Lacorre, D.A., Jain, S.R., Kozin, S. V., Stylianopoulos, T., Mousa, A.S., Han, X., Adstamongkonkul, P., et al. (2013). Angiotensin inhibition enhances drug delivery and potentiates chemotherapy by decompressing tumour blood vessels. Nat. Commun. 4.

Chen, G., Tian, X., Liu, Z., Zhou, S., Schmidt, B., Henne-Bruns, D., Bachem, M., and Kornmann, M. (2010). Inhibition of endogenous SPARC enhances pancreatic cancer cell growth: Modulation by FGFR1-III isoform expression. Br. J. Cancer 102, 188-195.

Chen, J., Wang, M., Xi, B., Xue, J., He, D., Zhang, J., and Zhao, Y. (2012). SPARC is a key regulator of proliferation, apoptosis and invasion in human ovarian cancer. PLoS One.

Chlenski, A., Liu, S., Crawford, S.E., Volpert, O. V., DeVries, G.H., Evangelista, A., Yang, Q., Salwen, H.R., Farrer, R., Bray, J., et al. (2002). SPARC is a key Schwannianderived inhibitor controlling neuroblastoma tumor angiogenesis. Cancer Res.

Chlenski, A., Guerrero, L.J., Peddinti, R., Spitz, J.A., Leonhardt, P.T., Yang, Q., Tian, Y., Salwen, H.R., and Cohn, S.L. (2010). Anti-angiogenic SPARC peptides inhibit progression of neuroblastoma tumors. Mol. Cancer 9, 1-10.

Chu, G.C., Kimmelman, A.C., Hezel, A.F., and DePinho, R.A. (2007). Stromal biology of pancreatic cancer. J. Cell. Biochem. 101, 887-907.

Clark, C.E., Hingorani, S.R., Mick, R., Combs, C., Tuveson, D.A., and Vonderheide, R.H. (2007). Dynamics of the immune reaction to pancreatic cancer from inception to invasion. Cancer Res. 67, 9518-9527.

Clezardin, P., Malaval, L., Ehrensperger, A. -S, DELMAS, P.D., DECHAVANNE, M., and MCGREGOR, J.L. (1988). Complex formation of human thrombospondin with osteonectin. Eur. J. Biochem. 
Collisson, E.A., Sadanandam, A., Olson, P., Gibb, W.J., Truitt, M., Gu, S., Cooc, J., Weinkle, J., Kim, G.E., Jakkula, L., et al. (2011). Subtypes of pancreatic ductal adenocarcinoma and their differing responses to therapy. Nat. Med. 17, 500-503.

Condeelis, J., and Pollard, J.W. (2006). Macrophages: Obligate partners for tumor cell migration, invasion, and metastasis. Cell 124, 263-266.

Conroy, T., Desseigne, F., Ychou, M., Bouche, O., Guimbaud, R., Becouarn, Y., Adenis, A., Raoul, J.L., Gourgou-Bourgade, S., de la Fouchardiere, C., et al. (2011). FOLFIRINOX versus gemcitabine for metastatic pancreatic cancer. N Engl J Med.

Cullinan, S.A., Moertel, C.G., Fleming, T.R., Rubin, J.R., Krook, J.E., Everson, L.K., Windschitl, H.E., Twito, D.I., Marschke, R.F., Foley, J.F., et al. (1985). A Comparison of Three Chemotherapeutic Regimens in the Treatment of Advanced Pancreatic and Gastric Carcinoma: Fluorouracil vs Fluorouracil and Doxorubicin vs Fluorouracil, Doxorubicin, and Mitomycin. JAMA J. Am. Med. Assoc.

Dangi-Garimella, S., Krantz, S.B., Barron, M.R., Shields, M.A., Heiferman, M.J., Grippo, P.J., Bentrem, D.J., and Munshi, H.G. (2011). Three-dimensional collagen I promotes gemcitabine resistance in pancreatic cancer through MT1-MMP-mediated expression of HMGA2. Cancer Res.

Dangi-Garimella, S., Sahai, V., Ebine, K., Kumar, K., and Munshi, H.G. (2013). ThreeDimensional Collagen I Promotes Gemcitabine Resistance In Vitro in Pancreatic Cancer Cells through HMGA2-Dependent Histone Acetyltransferase Expression. PLoS One 8, 1-9.

Demir, I.E., Friess, H., and Ceyhan, G.O. (2015). Neural plasticity in pancreatitis and pancreatic cancer. Nat. Rev. Gastroenterol. Hepatol.

Dhanesuan, N., Sharp, J.A., Blick, T., Price, J.T., and Thompson, E.W. (2002). Doxycycline-inducible expression of SPARC/Osteonectin/BM40 in MDA-MB-231 human breast cancer cells results in growth inhibition. Breast Cancer Res. Treat.

Diop-Frimpong, B., Chauhan, V.P., Krane, S., Boucher, Y., and Jain, R.K. (2011). Losartan inhibits collagen I synthesis and improves the distribution and efficacy of nanotherapeutics in tumors. Proc. Natl. Acad. Sci. 108, 2909-2914. 
Direkze, N.C., Hodivala-Dilke, K., Jeffery, R., Hunt, T., Poulsom, R., Oukrif, D., Alison, M.R., and Wright, N.A. (2004). Bone marrow contribution to tumor-associated myofibroblasts and fibroblasts. Cancer Res. 64, 8492-8495.

Downward, J. (2003). Role of receptor tyrosine kinases in G-protein-coupled receptor regulation of Ras: transactivation or parallel pathways? Biochem J 376, e9-10.

Ellenrieder, V., Alber, B., Lacher, U., Hendler, S.F., Menke, A., Boeck, W., Wagner, M., Wilda, M., Friess, H., Büchler, M., et al. (2000). Role of MT-MMPs and MMP-2 in pancreatic cancer progression. Int. J. Cancer 85, 14-20.

Engel, J., Paulsson, M., Taylor, W., Sage, H., and Hogan, B. (1987). Calcium Binding Domains and Calcium-Induced Conformational Transition of SPARC/BM40/Osteonectin, an Extracellular Glycoprotein Expressed in Mineralized and Nonmineralized Tissues. Biochemistry.

Erkan, M., Reiser-Erkan, C., Michalski, C.W., Deucker, S., Sauliunaite, D., Streit, S., Esposito, I., Friess, H., and Kleeff, J. (2009). Cancer-Stellate Cell Interactions Perpetuate the Hypoxia-Fibrosis Cycle in Pancreatic Ductal Adenocarcinoma. Neoplasia 11, 497508.

Esch, F.S., Shimasaki, S., Mercado, M., Cooksey, K., Ling, N., Ying, S., Ueno, N., and Guillemin, R. (1987). Structural Characterization of Follistatin: A Novel FollicleStimulating Hormone Release-Inhibiting Polypeptide from the Gonad. Mol. Endocrinol.

Esposito, I., Penzel, R., Chaib-Harrireche, M., Barcena, U., Bergmann, F., Riedl, S., Kayed, H., Giese, N., Kleeff, J., Friess, H., et al. (2006). Tenascin C and annexin II expression in the process of pancreatic carcinogenesis. J. Pathol. 208, 673-685.

Feig, C., Gopinathan, A., Neesse, A., Chan, D.S., Cook, N., and Tuveson, D.A. (2012). The pancreas cancer microenvironment. Clin. Cancer Res. 18, 4266-4276.

Fendrich, V., Esni, F., Garay, M.V.R., Feldmann, G., Habbe, N., Jensen, J.N., Dor, Y., Stoffers, D., Jensen, J., Leach, S.D., et al. (2008). Hedgehog Signaling Is Required for Effective Regeneration of Exocrine Pancreas. Gastroenterology. 
Fernanda Ledda, M., Adris, S., Bravo, A.I., Kairiyama, C., Bover, L., Chernajovsky, Y., Mordoh, J., and Podhajcer, O.L. (1997). Suppression of SPARC expression by antisense RNA abrogates the tumorigenicity of human melanoma cells. Nat. Med.

Fitzgerald, M.C., and Schwarzbauer, J.E. (1998). Importance of the basement membrane protein SPARC for viability and fertility in Caenorhabditis elegans. Curr. Biol.

Framson, P.E., and Sage, E.H. (2004). SPARC and tumor growth: Where the seed meets the soil? J. Cell. Biochem.

Francki, A., Bradshaw, A.D., Bassuk, J.A., Howe, C.C., Couser, W.G., and Sage, E.H. (1999a). SPARC regulates the expression of collagen type I and transforming growth factor- $\beta 1$ in mesangial cells. J. Biol. Chem. 274, 32145-32152.

Francki, a, Bradshaw, a D., Bassuk, J. a, Howe, C.C., Couser, W.G., and Sage, E.H. (1999b). SPARC regulates the expression of collagen type I and transforming growth factor-beta1 in mesangial cells. J. Biol. Chem.

Froeling, F.E.M., Feig, C., Chelala, C., Dobson, R., Mein, C.E., Tuveson, D.A., Clevers, H., Hart, I.R., and Kocher, H.M. (2011). Retinoic acid-induced pancreatic stellate cell quiescence reduces paracrine Wnt $\beta$-catenin signaling to slow tumor progression. Gastroenterology 141, 1486-1497.e14.

Funk, S.E., and Sage, E.H. (1991). The Ca2(+)-binding glycoprotein SPARC modulates cell cycle progression in bovine aortic endothelial cells. Proc. Natl. Acad. Sci. U. S. A.

Funk, S.E., and Sage, E.H. (1993). Differential effects of SPARC and cationic SPARC peptides on DNA synthesis by endothelial cells and fibroblasts. J. Cell. Physiol. 154, 5363.

Gao, J., Song, J., Huang, H., Li, Z., Du, Y., Cao, J., Li, M., Lv, S., Lin, H., and Gong, Y. (2010). Methylation of the SPARC gene promoter and its clinical implication in pancreatic cancer. J. Exp. Clin. Cancer Res. 29, 1-9.

Ghaneh, P., Costello, E., and Neoptolemos, J.P. (2008). Biology and management of pancreatic cancer. Postgrad. Med. J. 84, 478-497. 
Gilmour, D.T., Lyon, G.J., Carlton, M.B.L., Sanes, J.R., Cunningham, J.M., Anderson, J.R., Hogan, B.L.M., Evans, M.J., and Colledge, W.H. (1998). Mice deficient for the secreted glycoprotein SPARC/osteonectin/BM40 develop normally but show severe ageonset cataract formation and disruption of the lens. EMBO J. 17, 1860-1870.

Greiling, T.M.S., Stone, B., and Clark, J.I. (2009). Absence of SPARC leads to impaired lens circulation. Exp. Eye Res. 89, 416-425.

Gress, T.M., Menke, A., Bachem, M., Ellenrieder, V., Weidenbach, H., Wagner, M., and Adler, G. (1998). Role of Extracellular Matrix in pancreatic diseases. 625-637.

Guerra, C., Schuhmacher, A.J., Cañamero, M., Grippo, P.J., Verdaguer, L., PérezGallego, L., Dubus, P., Sandgren, E.P., and Barbacid, M. (2007). Chronic Pancreatitis Is Essential for Induction of Pancreatic Ductal Adenocarcinoma by K-Ras Oncogenes in Adult Mice. Cancer Cell 11, 291-302.

Guerra, C., Collado, M., Navas, C., Schuhmacher, A.J., Hernández-Porras, I., Cañamero, M., Rodriguez-Justo, M., Serrano, M., and Barbacid, M. (2011). Pancreatitis-induced inflammation contributes to pancreatic cancer by inhibiting oncogene-induced senescence. Cancer Cell 19, 728-739.

Guweidhi, A., Kleeff, J., Adwan, H., Giese, N.A., Wente, M.N., Giese, T., Büchler, M.W., Berger, M.R., and Friess, H. (2005). Osteonectin influences growth and invasion of pancreatic cancer cells. Ann. Surg. 242, 224-234.

Haber, C.L., Gottifredi, V., Llera, A.S., Salvatierra, E., Prada, F., Alonso, L., Helene, S.E., and Podhajcer, O.L. (2008). SPARC modulates the proliferation of stromal but not melanoma cells unless endogenous SPARC expression is downregulated. Int. J. Cancer.

Habisch, H., Zhou, S., Siech, M., and Bachem, M.G. (2010). Interaction of stellate cells with pancreatic carcinoma cells. Cancers (Basel). 2, 1661-1682.

Hahn, S. a, Schutte, M., Hoque, a T., Moskaluk, C. a, da Costa, L.T., Rozenblum, E., Weinstein, C.L., Fischer, A., Yeo, C.J., Hruban, R.H., et al. (1996). DPC4, a candidate tumor suppressor gene at human chromosome 18q21.1. Science (80-. ). 
Hambrock, H.O., Nitsche, D.P., Hansen, U., Bruckner, P., Paulsson, M., Maurer, P., and Hartmann, U. (2003). SC1/hevin: An extracellular calcium-modulated protein that binds collagen I. J. Biol. Chem.

Hambrock, H.O., Kaufmann, B., Müller, S., Hanisch, F.G., Nose, K., Paulsson, M., Maurer, P., and Hartmann, U. (2004). Structural characterization of TSC-36/Flik: Analysis of two charge isoforms. J. Biol. Chem.

Hao, N.B., Lü, M.H., Fan, Y.H., Cao, Y.L., Zhang, Z.R., and Yang, S.M. (2012). Macrophages in tumor microenvironments and the progression of tumors. Clin. Dev. Immunol. 2012.

Hasselaar, P., and Sage, E.H. (1992). SPARC antagonizes the effect of basic fibroblast growth factor on the migration of bovine aortic endothelial cells. J. Cell. Biochem.

Heinemann, V., Hertel, L., Grindey, G., and Plunkett, W. (1988). Comparison of the cellular pharmacokinetics and toxicity of $2^{\prime}, 2^{\prime}$-difluorodeoxycytidine and 1- $\beta$-darabinofuranosylcytosine. Cancer Res. 4024-4031.

Hessmann, E., Patzak, M.S., Klein, L., Chen, N., Kari, V., Ramu, I., Bapiro, T.E., Frese, K.K., Gopinathan, A., Richards, F.M., et al. (2018). Fibroblast drug scavenging increases intratumoural gemcitabine accumulation in murine pancreas cancer. Gut 67, 497-507.

Hidalgo, M., and Eckhardt, S.G. (2001). Development of matrix metalloproteinase inhibitors in cancer therapy. J. Natl. Cancer Inst. 93, 178-193.

Hingorani, S.R., Petricoin, E.F., Maitra, A., Rajapakse, V., King, C., Jacobetz, M.A., Ross, S., Conrads, T.P., Veenstra, T.D., Hitt, B.A., et al. (2003). Preinvasive and invasive ductal pancreatic cancer and its early detection in the mouse. Cancer Cell 4, 437-450.

Hingorani, S.R., Zheng, L., Bullock, A.J., Seery, T.E., Harris, W.P., Sigal, D.S., Braiteh, F., Ritch, P.S., Zalupski, M.M., Bahary, N., et al. (2018). HALO 202: Randomized phase II Study of PEGPH20 Plus Nab-Paclitaxel/Gemcitabine Versus NabPaclitaxel/Gemcitabine in Patients With Untreated, Metastatic Pancreatic Ductal Adenocarcinoma. J. Clin. Oncol. 36, 359-366.

Von Hoff, D.D., Ervin, T., Arena, F.P., Chiorean, E.G., Infante, J., Moore, M., Seay, T., Tjulandin, S.A., Ma, W.W., Saleh, M.N., et al. (2013). Increased survival in pancreatic 
cancer with nab-paclitaxel plus gemcitabine. N Engl J Med.

Hohenester, E., Maurer, P., Hohenadl, C., Timpl, R., Jansonius, J.N., and Engel, J. (1996). Structure of a novel extracellular Ca2+-binding module in BM-40. Nat. Struct. Biol.

Hohenester, E., Maurer, P., and Timpl, R. (1997). Crystal structure of a pair of follistatinlike and EF-hand calcium-binding domains in BM-40. EMBO J.

Howlader, N., Am, N., M, K., J, G., N, N., Sf, A., Cl, K., M, Y., J, R., Z, T., et al. (2012). SEER Cancer statistics review 1975-2010. J. Natl. Cancer Inst. 1-109.

Hruban, R.H., Takaori, K., Klimstra, D.S., Adsay, N.V., Albores-Saavedra, J., Biankin, A. V., Biankin, S.A., Compton, C., Fukushima, N., Furukawa, T., et al. (2004). An illustrated consensus on the classification of pancreatic intraepithelial neoplasia and intraductal papillary mucinous neoplasms. Am. J. Surg. Pathol. 28, 977-987.

Hwang, R.F., Moore, T., Arumugam, T., Ramachandran, V., Amos, K.D., Rivera, A., Ji, B., Evans, D.B., and Logsdon, C.D. (2008). Cancer-associated stromal fibroblasts promote pancreatic tumor progression. Cancer Res. 68, 918-926.

Iacobuzio-Donahue, C.A., Ashfaq, R., Maitra, A., Adsay, N.V., Shen-Ong, G.L., Berg, K., Hollingsworth, M.A., Cameron, J.L., Yeo, C.J., Kern, S.E., et al. (2003). Highly Expressed Genes in Pancreatic Ductal Adenocarcinomas: A Comprehensive Characterization and Comparison of the Transcription Profiles Obtained from Three Major Technologies. Cancer Res. 63, 8614-8622.

Ijichi, H., Chytil, A., Gorska, A.E., Aakre, M.E., Fujitani, Y., Fujitani, S., Wright, C.V.E., and Moses, H.L. (2006). Aggressive pancreatic ductal adenocarcinoma in mice caused by pancreas-specific blockade of transforming growth factor- $\beta$ signaling in cooperation with active Kras expression. Genes Dev. 20,3147-3160.

Incio, J., Liu, H., Suboj, P., Chin, S.M., Chen, I.X., Pinter, M., Ng, M.R., Nia, H.T., Grahovac, J., Kao, S., et al. (2016). Obesity-induced inflammation and desmoplasia promote pancreatic cancer progression and resistance to chemotherapy. Cancer Discov. 6, 852-869.

Infante, J.R., Matsubayashi, H., Sato, N., Tonascia, J., Klein, A.P., Riall, T.A., Yeo, C., 
Iacobuzio-Donahue, C., and Goggins, M. (2007). Peritumoral fibroblast SPARC expression and patient outcome with resectable pancreatic adenocarcinoma. J. Clin. Oncol. 25, 319-325.

Ingham, K.C., Brew, S.A., and Migliorini, M. (2002). Type I collagen contains at least 14 cryptic fibronectin binding sites of similar affinity. Arch. Biochem. Biophys.

Iodice, S., Gandini, S., Istituto, I.E.O., Istituto, I.E.O., Iodice, S., Gandini, S., and Maisonneuve, P. (2008). Tobacco and the Risk of Pancreatic Cancer: A Review and Meta-Analysis Tobacco and the risk of pancreatic cancer : a review and meta-analysis.

Ishii, G., Sangai, T., Oda, T., Aoyagi, Y., Hasebe, T., Kanomata, N., Endoh, Y., Okumura, C., Okuhara, Y., Magae, J., et al. (2003). Bone-marrow-derived myofibroblasts contribute to the cancer-induced stromal reaction. Biochem. Biophys. Res. Commun. 309, 232-240.

Jabłońska-Trypuć, A., Matejczyk, M., and Rosochacki, S. (2016). Matrix metalloproteinases (MMPs), the main extracellular matrix (ECM) enzymes in collagen degradation, as a target for anticancer drugs. J. Enzyme Inhib. Med. Chem. 31, 177-183.

Jackson, E.L., Willis, N., Mercer, K., Bronson, R.T., Crowley, D., Montoya, R., Jacks, T., and Tuveson, D.A. (2001). Analysis of lung tumor initiation and progression using conditional expression of oncogenic K-ras. 3243-3248.

Jacobetz, M.A., Chan, D.S., Neesse, A., Bapiro, T.E., Cook, N., Frese, K.K., Feig, C., Nakagawa, T., Caldwell, M.E., Zecchini, H.I., et al. (2013). Hyaluronan impairs vascular function and drug delivery in a mouse model of pancreatic cancer. Gut 62, 112-120.

Januchowski, R., Świerczewska, M., Sterzyńska, K., Wojtowicz, K., Nowicki, M., and Zabel, M. (2016). Increased expression of several collagen genes is associated with drug resistance in ovarian cancer cell lines. J. Cancer. 
Jones, S., Zhang, X., Parsons, D.W., Lin, J.C.-H., Leary, R.J., Angenendt, P., Mankoo, P., Carter, H., Kamiyama, H., Jimeno, A., et al. (2008). Core Signaling Pathways in Human Pancreatic Cancers Revealed by Global Genomic Analyses. Science (80-. ). 321, 1801-1806.

Kalluri, R., and Zeisberg, M. (2006). Fibroblasts in cancer. Nat. Rev. Cancer 6, 392-401.

Kanda, M., Matthaei, H., Wu, J., Hong, S.M., Yu, J., Borges, M., Hruban, R.H., Maitra, A., Kinzler, K., Vogelstein, B., et al. (2012). Presence of somatic mutations in most earlystage pancreatic intraepithelial neoplasia. Gastroenterology 142, 730-733.

Kanji, Z.S., Edwards, A.M., Mandelson, M.T., Sahar, N., Lin, B.S., Badiozamani, K., Song, G., Alseidi, A., Biehl, T.R., Kozarek, R.A., et al. (2018). Gemcitabine and Taxane Adjuvant Therapy with Chemoradiation in Resected Pancreatic Cancer: A Novel Strategy for Improved Survival? Ann. Surg. Oncol. 25, 1052-1060.

Kanno, A., Satoh, K., Masamune, A., Hirota, M., Kimura, K., Umino, J., Hamada, S., Satoh, A., Egawa, S., Motoi, F., et al. (2008). Periostin, secreted from stromal cells, has biphasic effect on cell migration and correlates with the epithelial to mesenchymal transition of human pancreatic cancer cells. Int. J. Cancer 122, 2707-2718.

Kano, M.R., Bae, Y., Iwata, C., Morishita, Y., Yashiro, M., Oka, M., Fujii, T., Komuro, A., Kiyono, K., Kaminishi, M., et al. (2007). Improvement of cancer-targeting therapy, using nanocarriers for intractable solid tumors by inhibition of TGF-beta signaling. Proc. Natl. Acad. Sci. 104, 3460-3465.

Kelm, R.J., and Mann, K.G. (1991). The collagen binding specificity of bone and platelet osteonectin is related to differences in glycosylation. J. Biol. Chem.

Khan, S., Ebeling, M.C., Chauhan, N., Thompson, P.A., Gara, R.K., Ganju, A., Yallapu, M.M., Behrman, S.W., Zhao, H., Zafar, N., et al. (2016). Sensitivity of Gemcitabine in Pancreatic Cancer. 75, 2292-2304.

Kikuta, K., Masamune, A., Watanabe, T., Ariga, H., Itoh, H., Hamada, S., Satoh, K., Egawa, S., Unno, M., and Shimosegawa, T. (2010). Pancreatic stellate cells promote epithelial-mesenchymal transition in pancreatic cancer cells. Biochem Biophys Res Commun. 
Klimstra, D.S., and Longnecker, D.S. (1994). K-ras mutations in pancreatic ductal proliferative lesions. Am. J. Pathol. 145, 1547-1550.

Knudsen, E.S., Vail, P., Balaji, U., Ngo, H., Botros, I.W., Makarov, V., Riaz, N., Balachandran, V., Leach, S., Thompson, D.M., et al. (2017). Stratification of pancreatic ductal adenocarcinoma: Combinatorial genetic, stromal, and immunologic markers. Clin. Cancer Res.

Koblinski, J.E., Kaplan-Singer, B.R., VanOsdol, S.J., Wu, M., Engbring, J.A., Wang, S., Goldsmith, C.M., Piper, J.T., Vostal, J.G., Harms, J.F., et al. (2005). Endogenous osteonectin/SPARC/BM-40 expression inhibits MDA-MB-231 breast cancer cell metastasis. Cancer Res.

Koukourakis, M.I., Giatromanolaki, A., Brekken, R.A., Sivridis, E., Gatter, K.C., Harris, A.L., and Sage, E.H. (2003). Enhanced expression of SPARC/osteonectin in the tumorassociated stroma of non-small cell lung cancer is correlated with markers of hypoxia/acidity and with poor prognosis of patients. Cancer Res.

Kupprion, C., Motamed, K., and Sage, E.H. (1998). SPARC (BM-40, osteonectin) inhibits the mitogenic effect of vascular endothelial growth factor on microvascular endothelial cells. J. Biol. Chem.

Lane, T.F., and Sage, E.H. (1994). The biology of SPARC, a protein that modulates cellmatrix interactions. FASEB J.

Lane, T.F., Iruela-Arispe, M.L., and Sage, E.H. (1992). Regulation of gene expression by SPARC during angiogenesis in vitro. Changes in fibronectin, thrombospondin-1, and plasminogen activator inhibitor-1. J. Biol. Chem.

Lane T. F and Sage Helene. (1994). The Biology of SPARC, a protein that modulates cell-matrix interactions. 121-144.

Lauth, M., Bergström, Å., Shimokawa, T., Tostar, U., Jin, Q., Fendrich, V., Guerra, C., Barbacid, M., and Toftgrd, R. (2010). DYRK1B-dependent autocrine-to-paracrine shift of Hedgehog signaling by mutant RAS. Nat. Struct. Mol. Biol. 
Lee, J.J., Perera, R.M., Wang, H., Wu, D.-C., Liu, X.S., Han, S., Fitamant, J., Jones, P.D., Ghanta, K.S., Kawano, S., et al. (2014). Stromal response to Hedgehog signaling restrains pancreatic cancer progression. Proc. Natl. Acad. Sci.

Leslie, K., Gao, S.P., Berishaj, M., Podsypanina, K., Ho, H., Ivashkiv, L., and Bromberg, J. (2010). Differential interleukin-6/Stat3 signaling as a function of cellular context mediates Ras-induced transformation. Breast Cancer Res.

Lillie, R.D., Tracy, R.E., Pizzolato, P., Donaldson, P.T., and Reynolds, C. (1980). Differential staining of collagen types in paraffin sections: A color change in degraded forms. Virchows Arch. A Pathol. Anat. Histol. 386, 153-159.

Liu, P., Lu, J., Cardoso, W. V, and Vaziri, C. (2008). The SPARC-related factor SMOC2 promotes growth factor-induced cyclin D1 expression and DNA synthesis via integrinlinked kinase. Mol. Biol. Cell.

Lohr, M., Schmidt, C., Kluth, M., Mu, P., Nizze, H., and Jesnowski, R. (2001). Transforming Growth Factor- NL 1 Induces Desmoplasia in an Experimental Model of Human Pancreatic Carcinoma 1. Cancer Res. 550-555.

Lokeshwar, V.B., Obek, C., Soloway, M.S., and Block, N.L. (1997). Tumor-associated hyaluronic acid: a new sensitive and specific urine marker for bladder cancer. Cancer Res. 57, 773-777.

Lowenfels, A.B., and Maisonneuve, P. (2004). Epidemiology and prevention of pancreatic cancer. Jpn.J.Clin.Oncol. 34, 238-244.

Määttä, M., Soini, Y., Liakka, A., and Autio-Harmainen, H. (2000). Differential expression of matrix metalloproteinase (MMP)-2, MMP-9, and membrane type 1-MMP in hepatocellular and pancreatic adenocarcinoma: implications for tumor progression and clinical prognosis. Clin. Cancer Res. 6, 2726-2734.

Mahlbacher, V., Sewing, A., Elsässer, H.P., and Kern, H.F. (1992). Hyaluronan is a secretory product of human pancreatic adenocarcinoma cells. Eur. J. Cell Biol.

Maisonneuve, P., and Lowenfels, A.B. (2010). Epidemiology of pancreatic cancer: An update. Dig. Dis. 28, 645-656. 
Mantoni, T.S., Schendel, R.R.E., Rödel, F., Al-Assar, O., Al-Assar, G.N.O., Masamune, A., and Brunner, T.B. (2008). Stromal SPARC expression and patient survival after chemoradiation for non-resectable pancreatic adenocarcinoma. Cancer Biol. Ther. 7, 1806-1815.

Martinek, N., Zou, R., Berg, M., Sodek, J., and Ringuette, M. (2002). Evolutionary conservation and association of SPARC with the basal lamina in Drosophila. Dev. Genes Evol. 212, 124-133.

Martinek, N., Shahab, J., Sodek, J., and Ringuette, M. (2006). Is SPARC an Evolutionarily Conserved Collagen Chaperone ? 296-305.

Martinek, N., Shahab, J., Saathoff, M., and Ringuette, M. (2011). Haemocyte-derived SPARC is required for collagen-IV-dependent stability of basal laminae in Drosophila embryos. J. Cell Sci.

Matthaei, H., Schulick, R.D., Hruban, R.H., and Maitra, A. (2011). Cystic precursors to invasive pancreatic cancer. Nat. Rev. Gastroenterol. Hepatol. 8, 141-150.

Maurer, P., and Hohenester, E. (1997). Structural and functional aspects of calcium binding in extracellular matrix proteins. Matrix Biol.

Maurer, P., Mayer, U., Bruch, M., Jenö, P., Mann, K., Landwehr, R., Engel, J., and Timpl, R. (1992). High-affinity and low-affinity calcium binding and stability of the multidomain extracellular $40-\mathrm{kDa}$ basement membrane glycoprotein (BM40/SPARC/osteonectin). Eur. J. Biochem.

Mccleary-Wheeler, A.L., Mcwilliams, R., and Fernandez-Zapico, M.E. (2012). Aberrant signaling pathways in pancreatic cancer: A two compartment view. Mol. Carcinog. 51, 25-39.

Miller, B.W., Morton, J.P., Pinese, M., Saturno, G., Jamieson, N.B., McGhee, E., Timpson, P., Leach, J., McGarry, L., Shanks, E., et al. (2015). Targeting the LOX/hypoxia axis reverses many of the features that make pancreatic cancer deadly: inhibition of LOX abrogates metastasis and enhances drug efficacy. EMBO Mol. Med. 7, 1063-1076. 
Minchinton, A.I., and Tannock, I.F. (2006). Drug penetration in solid tumours. Nat. Rev. Cancer 6, 583-592.

Mini, E., Nobili, S., Caciagli, B., Landini, I., and Mazzei, T. (2006). Cellular pharmacology of gemcitabine. Ann. Oncol. 17, 7-12.

Mizuguchi, Y., Yoshida, H., Yokomuro, S., Arima, Y., Mamada, Y., Taniai, N., Akimaru, K., and Tajiri, T. (2005). Collagen IV is a predictor for clinical course in patients with malignant obstructive jaundice. Hepatogastroenterology.

Moffitt, R.A., Marayati, R., Flate, E.L., Volmar, K.E., Loeza, S.G.H., Hoadley, K.A., Rashid, N.U., Williams, L.A., Eaton, S.C., Chung, A.H., et al. (2015). Virtual microdissection identifies distinct tumor- and stroma-specific subtypes of pancreatic ductal adenocarcinoma. Nat. Genet. 47, 1168-1178.

Morris et al. (2010). KRAS, Hedgehog, Wnt and the twisted developmental biology of pancreatic ductal adenocarcinoma John. 292, 342-351.

Motamed, K., and Sage, E.H. (1998). SPARC inhibits endothelial cell adhesion but not proliferation through a tyrosine phosphorylation-dependent pathway. J. Cell. Biochem.

Murphy, K.M., Brune, K.A., Griffin, C., Sollenberger, J.E., Petersen, G.M., Bansal, R., Hruban, R.H., and Kern, S.E. (2002). Evaluation of candidate genes MAP2K4, MADH4, ACVR1B, and BRCA2 in familial pancreatic cancer: deleterious BRCA2 mutations in 17\%. Cancer Res. 62, 3789-3793.

Nagaraju, G.P., Dontula, R., El-rayes, B.F., and Lakka, S.S. (2014). Molecular mechanisms underlying the divergent roles of SPARC in human carcinogenesis. Carcinogenesis 35, 967-973.

Neesse, A., Michl, P., Frese, K.K., Feig, C., Cook, N., Jacobetz, M.A., Lolkema, M.P., Buchholz, M., Olive, K.P., Gress, T.M., et al. (2011). Stromal biology and therapy in pancreatic cancer. Gut 60, 861-868.

Neesse, A., Frese, K.K., Bapiro, T.E., Nakagawa, T., Sternlicht, M.D., Seeley, T.W., Pilarsky, C., Jodrell, D.I., Spong, S.M., and Tuveson, D.A. (2013). CTGF antagonism with mAb FG-3019 enhances chemotherapy response without increasing drug delivery in murine ductal pancreas cancer. Proc. Natl. Acad. Sci. 110, 12325-12330. 
Neesse, A., Frese, K.K., Chan, D.S., Bapiro, T.E., Howat, W.J., Richards, F.M., Ellenrieder, V., Jodrell, D.I., and Tuveson, D.A. (2014). SPARC independent drug delivery and antitumour effects of nab -paclitaxel in genetically engineered mice. 974983.

Neesse, A., Algül, H., Tuveson, D.A., and Gress, T.M. (2015). Stromal biology and therapy in pancreatic cancer: A changing paradigm. Gut 64, 1476-1484.

Neoptolemos, J.P., Stocken, D.D., Bassi, C., Ghaneh, P., Cunningham, D., Goldstein, D., Padbury, R., Moore, M.J., Gallinger, S., Mariette, C., et al. (2010). Adjuvant Chemotherapy With Fluorouracil Plus Folinic Acid vs Gemcitabine Following Pancreatic Cancer Resection. September 304, 1073-1081.

Neoptolemos, J.P., Palmer, D.H., Ghaneh, P., Psarelli, E.E., Valle, J.W., Halloran, C.M., Faluyi, O., O’Reilly, D.A., Cunningham, D., Wadsley, J., et al. (2017). Comparison of adjuvant gemcitabine and capecitabine with gemcitabine monotherapy in patients with resected pancreatic cancer (ESPAC-4): a multicentre, open-label, randomised, phase 3 trial. Lancet 389, 1011-1024.

Noël, A., Jost, M., and Maquoi, E. (2008). Matrix metalloproteinases at cancer tumorhost interface. Semin. Cell Dev. Biol.

Norose, K., Clark, J.I., Syed, N.A., Basu, A., Heber-Katz, E., Sage, E.H., and Howe, C.C. (1998a). SPARC deficiency leads to early-onset cataractogenesis. Investig. Ophthalmol. Vis. Sci. 39, 2674-2680.

Norose, K., Clark, J.I., Syed, N.A., Basu, A., Heber-Katz, E., Sage, E.H., and Howe, C.C. (1998b). SPARC deficiency leads to early-onset cataractogenesis. Investig. Ophthalmol. Vis. Sci.

Oettle, H., Neuhaus, P., Hochhaus, A., Hartmann, J.T., Gellert, K., Ridwelski, K., Niedergethmann, M., Zülke, C., Fahlke, J., Arning, M.B., et al. (2013). Adjuvant Chemotherapy With Gemcitabine and Long-term Outcomes Among Patients With Resected Pancreatic Cancer. Jama 310, 1473.

Oettle et al. (2007). Adjuvant Chemotherapy With Gemcitabine vs Observation in Patients Undergoing Curative-Intent Resection of Pancreatic Cancer A Randomized Controlled Trial. Database 297, 267-277. 
Öhlund, D., Elyada, E., and Tuveson, D. (2014). Fibroblast heterogeneity in the cancer wound. J. Exp. Med.

Öhlund, D., Handly-Santana, A., Biffi, G., Elyada, E., Almeida, A.S., Ponz-Sarvise, M., Corbo, V., Oni, T.E., Hearn, S.A., Lee, E.J., et al. (2017). Distinct populations of inflammatory fibroblasts and myofibroblasts in pancreatic cancer. J. Exp. Med. jem.20162024.

Olive, K.P., Jacobetz, M.A., Davidson, C.J., Gopinathan, A., McIntyre, D., Honess, D., Madhu, B., Goldgraben, M.A., Caldwell, M.E., Allard, D., et al. (2009). Inhibition of Hedgehog Signaling Enhances Delivery of Chemotherapy in a Mouse Model of Pancreatic Cancer. Science (80-. ). 324, 1457-1461.

Ozawa, F., Friess, H., and Caliera, a T. (2001). Growth factors and their receptors in pancreatic cancer - Ozawa - 2000 - Teratogenesis, Carcinogenesis, and Mutagenesis Wiley Online Library. Teratogenesis 44, 27-44.

Özdemir, B.C., Pentcheva-Hoang, T., Carstens, J.L., Zheng, X., Wu, C.C., Simpson, T.R., Laklai, H., Sugimoto, H., Kahlert, C., Novitskiy, S. V., et al. (2014). Depletion of carcinoma-associated fibroblasts and fibrosis induces immunosuppression and accelerates pancreas cancer with reduced survival. Cancer Cell 25, 719-734.

Parkin, D.., Bray, F.., and Devesa, S.. (2001). Cancer burden in the year 2000. The global picture. Eur. J. Cancer.

Patthy, L. (1991). Modular exchange principles in proteins. Curr. Opin. Struct. Biol.

Phillips, P.A., McCarroll, J.A., Park, S., Wu, M.-J., Pirola, R., Korsten, M., Wilson, J.S., and Apte, M. V (2003). Rat pancreatic stellate cells secrete matrix metalloproteinases: implications for extracellular matrix turnover. Gut.

Pickart, L., and Lovejoy, S. (1987). Biological Activity of Human Plasma CopperBinding Growth Factor Glycyl-L-Histidyl-L-Lysine. Methods Enzymol.

Pickart, L., Freedman, J.H., Loker, W.J., Peisach, J., Perkins, C.M., Stenkamp, R.E., and Weinstein, B. (1980). Growth-modulating plasma tripeptide may function by facilitating copper uptake into cells. Nature 288, 715-717. 
Pollard, J.W. (2004). Opinion: Tumour-educated macrophages promote tumour progression and metastasis. Nat. Rev. Cancer 4, 71-78.

Poschl, E. (2004). Collagen IV is essential for basement membrane stability but dispensable for initiation of its assembly during early development. Development.

Provenzano, P.P., Cuevas, C., Chang, A.E., Goel, V.K., Von Hoff, D.D., and Hingorani, S.R. (2012). Enzymatic Targeting of the Stroma Ablates Physical Barriers to Treatment of Pancreatic Ductal Adenocarcinoma. Cancer Cell 21, 418-429.

Puolakkainen, P. a, Brekken, R. a, Muneer, S., and Sage, E.H. (2004). Enhanced growth of pancreatic tumors in SPARC-null mice is associated with decreased deposition of extracellular matrix and reduced tumor cell apoptosis. Mol. Cancer Res. 2, 215-224.

Pylayeva-Gupta, Y., Lee, K.E., Hajdu, C.H., Miller, G., and Bar-Sagi, D. (2012). Oncogenic Kras-Induced GM-CSF Production Promotes the Development of Pancreatic Neoplasia. Cancer Cell.

Qian, X., Rothman, V.L., Nicosia, R.F., and Tuszynski, G.P. (2001). Expression of thrombospondin-1 in human pancreatic adenocarcinomas: Role in matrix metalloproteinase-9 production. Pathol. Oncol. Res. 7, 251-259.

Rahib, L., Smith, B.D., Aizenberg, R., Rosenzweig, A.B., Fleshman, J.M., and Matrisian, L.M. (2014). Projecting cancer incidence and deaths to 2030: The unexpected burden of thyroid, liver, and pancreas cancers in the united states. Cancer Res. 74, 2913-2921.

Raines, E.W., Lane, T.F., Iruela-Arispe, M.L., Ross, R., and Sage, E.H. (1992). The extracellular glycoprotein SPARC interacts with platelet-derived growth factor (PDGF)$\mathrm{AB}$ and $-\mathrm{BB}$ and inhibits the binding of PDGF to its receptors. Proc. Natl. Acad. Sci. U. S. A.

Rhim, A.D., Oberstein, P.E., Thomas, D.H., Mirek, E.T., Palermo, C.F., Sastra, S.A., Dekleva, E.N., Saunders, T., Becerra, C.P., Tattersall, I.W., et al. (2014). Stromal elements act to restrain, rather than support, pancreatic ductal adenocarcinoma. Cancer Cell 25, 735-747.

Rizzato, C., Campa, D., Pezzilli, R., Soucek, P., Greenhalf, W., Capurso, G., Talarwojnarowska, R., Heller, A., Jamroziak, K., Khaw, K., et al. (2013). ABO blood groups 
and pancreatic cancer risk and survival : Results from the PANcreatic Disease ReseArch ( PANDoRA ) consortium. 1637-1644.

Robinson, E., Foty, R., and Corbett, S. (2004). Fibronectin Matrix Assembly Regulates alpha5beta1-mediated Cell Cohesion. Mol. Biol. Cell.

Romberg, R.W., Werness, P.G., Lollar, P., Riggs, B.L., and Manns, K.G. (1985). Isolation and Characterization of Native Adult Osteonectin\&quot; J. Biol. Chem.

Rosenblatt, S., Bassuk, J. a, Alpers, C.E., Sage, E.H., Timpl, R., and Preissner, K.T. (1997). Differential modulation of cell adhesion by interaction between adhesive and counter-adhesive proteins: characterization of the binding of vitronectin to osteonectin (BM40, SPARC). Biochem. J.

Sage, H., Johnson, C., and Bornstein, P. (1984). Characterization of a novel serum albumin-binding glycoprotein secreted by endothelial cells in culture. J. Biol. Chem. 259, 3993-4007.

Sage, H., Vernon, R.B., Funk, S.E., Everitt, E.A., and Angello, J. (1989). SPARC, a secreted protein associated with cellular proliferation, inhibits cell spreading in vitro and exhibits $\mathrm{Ca}+2$-dependent binding to the extracellular matrix. J. Cell Biol.

Said, N., Socha, M.J., Olearczyk, J.J., Elmarakby, A.A., Imig, J.D., and Motamed, K. (2007). Normalization of the Ovarian Cancer Microenvironment by SPARC. Mol. Cancer Res. 5, 1015-1030.

Said, N., Frierson, H.F., Sanchez-Carbayo, M., Brekken, R.A., and Theodorescu, D. (2013). Loss of SPARC in bladder cancer enhances carcinogenesis and progression. J. Clin. Invest. 123, 751-766.

Sato, N., Fukushima, N., Maehara, N., Matsubayashi, H., Koopmann, J., Su, G.H., Hruban, R.H., and Goggins, M. (2003). SPARC/osteonectin is a frequent target for aberrant methylation in pancreatic adenocarcinoma and a mediator of tumor-stromal interactions. Oncogene 22, 5021-5030. 
Sato, N., Kohi, S., Hirata, K., and Goggins, M. (2016). Role of hyaluronan in pancreatic cancer biology and therapy: Once again in the spotlight. Cancer Sci.

Schäfer, H., Geismann, C., Heneweer, C., Egberts, J.H., Korniienko, O., Kiefel, H., Moldenhauer, G., Bachem, M.G., Kalthoff, H., Altevogt, P., et al. (2012). Myofibroblastinduced tumorigenicity of pancreatic ductal epithelial cells is L1CAM dependent. Carcinogenesis 33, 84-93.

Schiemann, B.J., Neil, J.R., and Schiemann, W.P. (2003). SPARC inhibits epithelial cell proliferation in part through stimulation of the transforming growth factor-beta-signaling system. Mol. Biol. Cell.

Schneider, G., Siveke, J.T., Eckel, F., and Schmid, R.M. (2005). Pancreatic cancer: Basic and clinical aspects. Gastroenterology 128, 1606-1625.

Schneiderhan, W., Diaz, F., Fundel, M., Zhou, S., Siech, M., Hasel, C., Moller, P., Gschwend, J.E., Seufferlein, T., Gress, T., et al. (2007). Pancreatic stellate cells are an important source of MMP-2 in human pancreatic cancer and accelerate tumor progression in a murine xenograft model and CAM assay. J. Cell Sci. 120, 512-519.

Schnepp, A., Lindgren, P.K., Hülsmann, H., Kröger, S., Paulsson, M., and Hartmann, U. (2005). Mouse testican-2: Expression, glycosylation, and effects on neurite outgrowth. J. Biol. Chem.

Schubbert, S., Shannon, K., and Bollag, G. (2007). Hyperactive Ras in developmental disorders and cancer. Nat. Rev. Cancer 7, 295-308.

Shek, F.W., Benyon, R.C., Walker, F.M., McCrudden, P.R., Pender, S.L., Williams, E.J., Johnson, P.A., Johnson, C.D., Bateman, A.C., Fine, D.R., et al. (2002). Expression of transforming growth factor-beta 1 by pancreatic stellate cells and its implications for matrix secretion and turnover in chronic pancreatitis. Am J Pathol 160, 1787-1798.

Sherman, M.H., Yu, R.T., Engle, D.D., Ding, N., Atkins, A.R., Tiriac, H., Collisson, E.A., Connor, F., Van Dyke, T., Kozlov, S., et al. (2014). Vitamin D receptor-mediated stromal reprogramming suppresses pancreatitis and enhances pancreatic cancer therapy. Cell 159, 80-93. 
Sherman-Baust, C.A., Weeraratna, A.T., Rangel, L.B.A., Pizer, E.S., Cho, K.R., Schwartz, D.R., Shock, T., and Morin, P.J. (2003). Remodeling of the extracellular matrix through overexpression of collagen VI contributes to cisplatin resistance in ovarian cancer cells. Cancer Cell.

Shi, C., Hruban, R.H., and Klein, A.P. (2009). Familial pancreatic cancer. Arch. Pathol. Lab. Med. 133, 365-374.

Shin, M., Mizokami, A., Kim, J., Ofude, M., Konaka, H., Kadono, Y., Kitagawa, Y., Miwa, S., Kumaki, M., Keller, E.T., et al. (2013). Exogenous SPARC suppresses proliferation and migration of prostate cancer by interacting with integrin $\beta 1$. Prostate.

Siegel, R., Miller, K.D., and Ahmedin, J. (2017). Cancer Statistics 2017. CA CANCER J CLIN 2017 67, 7-30.

Singha, N.C., Nekoroski, T., Zhao, C., Symons, R., Jiang, P., Frost, G.I., Huang, Z., and Shepard, H.M. (2015). Tumor-Associated Hyaluronan Limits Efficacy of Monoclonal Antibody Therapy. Mol. Cancer Ther. 14, 523-532.

Sinn, M., Sinn, B. V., Striefler, J.K., Lindner, J.L., Stieler, J.M., Lohneis, P., Bischoff, S., Bläker, H., Pelzer, U., Bahra, M., et al. (2014). SPARC expression in resected pancreatic cancer patients treated with gemcitabine: results from the CONKO-001 study. Ann. Oncol. 25, 1025-1032.

Sinn, M., Bahra, M., Liersch, T., Gellert, K., Messmann, H., Bechstein, W., Waldschmidt, D., Jacobasch, L., Wilhelm, M., Rau, B.M., et al. (2017). CONKO-005: Adjuvant chemotherapy with gemcitabine plus erlotinib versus gemcitabine alone in patients after $\mathrm{r} 0$ resection of pancreatic cancer: A multicenter randomized phase III trial. J. Clin. Oncol. 35, 3330-3337.

Sparmann, A., and Bar-Sagi, D. (2004). Ras-induced interleukin-8 expression plays a critical role in tumor growth and angiogenesis. Cancer Cell.

Stocken, D.D., Büchler, M.W., Dervenis, C., Bassi, C., Jeekel, H., Klinkenbijl, J.H.G., Bakkevold, K.E., Takada, T., Amano, H., and Neoptolemos, J.P. (2005). Meta-analysis of randomised adjuvant therapy trials for pancreatic cancer. Br. J. Cancer 92, 1372-1381. Sugimoto, H., Mundel, T.M., Kieran, M.W., and Kalluri, R. (2006). Identification of 
fibroblast heterogeneity in the tumor microenvironment. Cancer Biol. Ther.

Sullivan, M.M., and Sage, E.H. (2004). Hevin/SC1, a matricellular glycoprotein and potential tumor-suppressor of the SPARC/BM-40/Osteonectin family. Int. J. Biochem. Cell Biol.

Suzuki, M., Hao, C., Takahashi, T., Shigematsu, H., Shivapurkar, N., Sathyanarayana, U.G., Iizasa, T., Fujisawa, T., Hiroshima, K., and Gazdar, A.F. (2005). Aberrant methylation of SPARC in human lung cancers. Br. J. Cancer.

Termine, J.D., Kleinman, H.K., Whitson, S.W., Conn, K.M., McGarvey, M.L., and Martin, G.R. (1981). Osteonectin, a bone-specific protein linking mineral to collagen. Cell 26, 99-105.

Thayer, S.P., Di Magliano, M.P., Heiser, P.W., Nielsen, C.M., Roberts, D.J., Lauwers, G.Y., Qi, Y.P., Gysin, S., Fernández-del Castillo, C., Yajnik, V., et al. (2003). Hedgehog is an early and late mediator of pancreatic cancer tumorigenesis. Nature.

Theocharis, A.D., Skandalis, S.S., Gialeli, C., and Karamanos, N.K. (2016). Extracellular matrix structure. Adv. Drug Deliv. Rev. 97, 4-27.

Thompson, D., and Easton, D.F. (2002). Cancer Incidence in BRCA1 mutation carriers. J. Natl. Cancer Inst. 94, 1358-1365.

Thompson, C.B., Shepard, H.M., O’Connor, P.M., Kadhim, S., Jiang, P., Osgood, R.J., Bookbinder, L.H., Li, X., Sugarman, B.J., Connor, R.J., et al. (2010). Enzymatic Depletion of Tumor Hyaluronan Induces Antitumor Responses in Preclinical Animal Models. Mol. Cancer Ther. 9, 3052-3064.

Tomotaki, S., Mizumoto, H., Hamabata, T., Kumakura, A., Shiota, M., Arai, H., Haginoya, K., and Hata, D. (2016). Severe Hemolytic Jaundice in a Neonate with a Novel COL4A1 Mutation. Pediatr. Neonatol.

Tramacere, I., Scotti, L., Jenab, M., Bagnardi, V., Bellocco, R., Rota, M., Corrao, G., Bravi, F., Boffetta, P., and Vecchia, C. La (2010). Alcohol drinking and pancreatic cancer risk: a meta-analysis of the dose-risk relation. 1486, 1474-1486. 
Tremble, P.M., Lane, T.F., Sage, E.H., and Werb, Z. (1993). SPARC, a secreted protein associated with morphogenesis and tissue remodeling, induces expression of metalloproteinases in fibroblasts through a novel extracellular matrix-dependent pathway. J. Cell Biol. 121, 1433-1444.

Turati, F., Edefonti, V., Bosetti, C., Ferraroni, M., Malvezzi, M., Franceschi, S., Talamini, R., Montella, M., Levi, F., Maso, L.D., et al. (2013). Family history of cancer and the risk of cancer: a network of case-control studies. Ann. Oncol. 24.

Tuveson, D.A., Zhu, L., Gopinathan, A., Willis, N.A., Kachatrian, L., Grochow, R., Pin, C.L., Mitin, N.Y., Taparowsky, E.J., Gimotty, P.A., et al. (2006). Mist1KrasG12Dknock-in mice develop mixed differentiation metastatic exocrine pancreatic carcinoma and hepatocellular carcinoma. Cancer Res. 66, 242-247.

Vannahme, C., Schübel, S., Herud, M., Gösling, S., Hülsmann, H., Paulsson, M., Hartmann, U., and Maurer, P. (1999). Molecular cloning of testican-2: Defining a novel calcium-binding proteoglycan family expressed in brain. J. Neurochem.

Vannahme, C., Smyth, N., Miosge, N., Gösling, S., Frie, C., Paulsson, M., Maurer, P., and Hartmann, U. (2002). Characterization of SMOC-1, a novel modular calciumbinding protein in basement membranes. J. Biol. Chem.

Vannahme, C., Gösling, S., Paulsson, M., Maurer, P., and Hartmann, U. (2003). Characterization of SMOC-2, a modular extracellular calcium-binding protein. Biochem. $\mathrm{J}$.

Vernon, R.B., and Sage, H. (1989). The calcium-binding protein SPARC is secreted by Leydig and Sertoli cells of the adult mouse testis. Biol Reprod.

Vonlaufen, A., Joshi, S., Qu, C., Phillips, P.A., Xu, Z., Parker, N.R., Toi, C.S., Pirola, R.C., Wilson, J.S., Goldstein, D., et al. (2008a). Pancreatic stellate cells: Partners in crime with pancreatic cancer cells. Cancer Res. 68, 2085-2093.

Vonlaufen, A., Phillips, P.A., Xu, Z., Goldstein, D., Pirola, R.C., Wilson, J.S., and Apte, M. V. (2008b). Pancreatic stellate cells and pancreatic cancer cells: An unholy alliance. Cancer Res. 
Waddell, N., Pajic, M., Patch, A.M., Chang, D.K., Kassahn, K.S., Bailey, P., Johns, A.L., Miller, D., Nones, K., Quek, K., et al. (2015). Whole genomes redefine the mutational landscape of pancreatic cancer. Nature $518,495-501$.

Wahi, M.M., Shah, N., Schrock, C.E., Rosemurgy, A.S., and Goldin, S.B. (2009). Reproductive Factors and Risk of Pancreatic Cancer in Women: A Review of the Literature. Ann. Epidemiol. 19, 103-111.

Wang, H., Fertala, A., Ratner, B.D., Sage, E.H., and Jiang, S. (2005). Identifying the SPARC binding sites on collagen I and procollagen I by atomic force microscopy. Anal. Chem. 77, 6765-6771.

Whatcott, C.J., Diep, C.H., Jiang, P., Watanabe, A., Lobello, J., Sima, C., Hostetter, G., Shepard, H.M., Von Hoff, D.D., and Han, H. (2015). Desmoplasia in primary tumors and metastatic lesions of pancreatic cancer. Clin. Cancer Res. 21, 3561-3568.

Witkiewicz, A.K., McMillan, E.A., Balaji, U., Baek, G.H., Lin, W.C., Mansour, J., Mollaee, M., Wagner, K.U., Koduru, P., Yopp, A., et al. (2015). Whole-exome sequencing of pancreatic cancer defines genetic diversity and therapeutic targets. Nat. Commun. 6.

WRANA, J.L., OVERALL, C.M., and SODEK, J. (1991). Regulation of the expression of a secreted acidic protein rich in cysteine (SPARC) in human fibroblasts by transforming growth factor $\beta$ : Comparison of transcriptional and post-transcriptional control with fibronectin and type I collagen. Eur. J. Biochem. 197, 519-528.

Xu, Y., Gurusiddappa, S., Rich, R.L., Owens, R.T., Keene, D.R., Mayne, R., Höök, a, and Höök, M. (2000). Multiple binding sites in collagen type I for the integrins alpha1beta1 and alpha2beta1. J. Biol. Chem.

Yahya, R.S., El-Bindary, A.A., El-Mezayen, H.A., Abdelmasseh, H.M., and Eissa, M.A. (2014). Biochemical evaluation of hyaluronic acid in breast cancer. Clin.Lab.

Yan, Q., and Sage, E.H. (1999). SPARC, a matricellular glycoprotein with important biological functions. J. Histochem. Cytochem. 47, 1495-1505. 
Yauch, R.L., Gould, S.E., Scales, S.J., Tang, T., Tian, H., Ahn, C.P., Marshall, D., Fu, L., Januario, T., Kallop, D., et al. (2008). A paracrine requirement for hedgehog signalling in cancer. Nature.

Yiu, G.K., Chan, W.Y., Ng, S.W., Chan, P.S., Cheung, K.K., Berkowitz, R.S., and Mok, S.C. (2001). SPARC (secreted protein acidic and rich in cysteine) induces apoptosis in ovarian cancer cells. Am. J. Pathol.

Yoshida, S., Yokota, T., Ujiki, M., Ding, X.Z., Pelham, C., Adrian, T.E., Talamonti, M.S., Bell, R.H.J., and Denham, W. (2004). Pancreatic cancer stimulates pancreatic stellate cell proliferation and TIMP-1 production through the MAP kinase pathway. Biochem Biophys Res Commun.

Yoshida, S., Ujiki, M., Ding, X.Z., Pelham, C., Talamonti, M.S., Bell, R.H., Denham, W., and Adrian, T.E. (2005). Pancreatic Stellate Cells (PSCs) express cyclooxygenase-2 (COX-2) and pancreatic cancer stimulates COX-2 in PSCs. Mol. Cancer.

Zhang, J.L., Chen, G.W., Liu, Y.C., Wang, P.Y., Wang, X., Wan, Y.L., Zhu, J., Gao, H.Q., Yin, J., Wang, W., et al. (2012). Secreted Protein Acidic and Rich in Cysteine (SPARC) Suppresses Angiogenesis by Down-Regulating the Expression of VEGF and MMP-7 in Gastric Cancer. PLoS One 7, 1-10.

Zhou, H.Y., Salih, E., and Glimcher, M.J. (1998). Isolation of a novel bone glycosylated phosphoprotein with disulphide cross-links to osteonectin. Biochem. J. 